PREMIO TESI DI DOTTORATO

ISSN 2612-8039 (PRINT) | ISSN 2612-8020 (ONLINE)

$-74-$ 
PREMIO TESI DI DOTTORATO

Commissione giudicatrice, anno 2018

Vincenzo Varano, Presidente della Commissione

Tito Arecchi, Area Scientifica

Aldo Bompani, Area delle Scienze Sociali

Mario Caciagli, Area delle Scienze Sociali

Franco Cambi, Area Umanistica

Paolo Felli, Area Tecnologica

Giancarlo Garfagnini, Area Umanistica

Roberto Genesio, Area Tecnologica

Flavio Moroni, Area Biomedica

Adolfo Pazzagli, Area Biomedica

Giuliano Pinto, Area Umanistica

Vincenzo Schettino, Area Scientifica

Luca Uzielli, Area Tecnologica

Graziella Vescovini, Area Umanistica 
Alessia Costa

\section{Histaminergic neurotransmission as a gateway for the effects of the fat sensing molecule Oleoylethanolamide}

Focus on cognition and stress-reactivity 


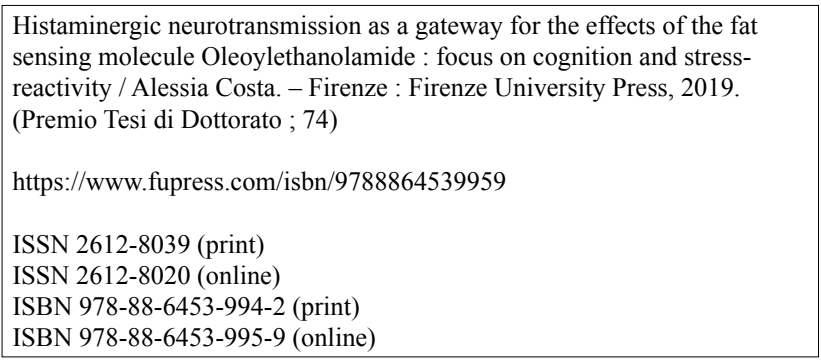

Graphic design: Alberto Pizarro Fernández, Lettera Meccanica SRLs

Peer Review Process

All publications are submitted to an external refereeing process under the responsibility of the FUP Editorial Board and the Scientific Committees of the individual series. The works published in the FUP catalogue are evaluated and approved by the Editorial Board of the publishing house. For a more detailed description of the refereeing process we refer to the official documents published on the website and in the online catalogue (www.fupress.com).

Firenze University Press Editorial Board

M. Garzaniti (Editor-in-Chief), M.E. Alberti, M. Boddi, A. Bucelli, R. Casalbuoni, A. Dolfi, R. Ferrise, M.C. Grisolia, P. Guarnieri, R. Lanfredini, P. Lo Nostro, G. Mari, A. Mariani, P.M. Mariano, S. Marinai, R. Minuti, P. Nanni, A. Orlandi, A. Perulli, G. Pratesi.

The online digital edition is published in Open Access on www.fupress.com.

Content license: the present work is released under Creative Commons Attribution 4.0 International license (CC BY 4.0: http://creativecommons.org/licenses/by/4.0/legalcode). This license allows you to share any part of the work by any means and format, modify it for any purpose, including commercial, as long as appropriate credit is given to the author, any changes made to the work are indicated and a URL link is provided to the license.

Metadata license: all the metadata are released under the Public Domain Dedication license (CC0 1.0 Universal: https://creativecommons.org/publicdomain/zero/1.0/legalcode).

(C) 2019 Author(s)

Published by Firenze University Press

Firenze University Press

Università degli Studi di Firenze

via Cittadella, 7, 50144 Firenze, Italy

www.fupress.com

This book is printed on acid-free paper

Printed in Italy 
A mamma, papà, Enrica ed Antonio 



\section{Index}

$\begin{array}{lr}\text { Abstract } & 12\end{array}$

\section{Chapter 1}

Histamine In The Nervous System $\quad 14$

$\begin{array}{ll}1.1 \text { Anatomic Framework } & 14\end{array}$

$\begin{array}{ll}1.2 \text { Histaminergic Receptors } & 15\end{array}$

1.2.1 Histamine $\mathrm{H}_{1}$ Receptor $\quad 15$

1.2.2. Histamine $\mathrm{H}_{2}$ Receptor $\quad 16$

$\begin{array}{ll}1.2 .3 \text { Histamine } \mathrm{H}_{3} \text { Receptor } & 16\end{array}$

$\begin{array}{ll}\text { 1.2.4 Histamine } \mathrm{H}_{4} \text { Receptor } & 17\end{array}$

$\begin{array}{ll}1.3 \text { Homeostatic Histaminergic Functions } & 17\end{array}$

$\begin{array}{ll}\text { 1.3.1 Sleep and Wakefulness } & 18\end{array}$

$\begin{array}{ll}\text { 1.3.2 Thermoregulation } & 18\end{array}$

$\begin{array}{ll}1.3 .3 \text { Fluid Balance } & 18\end{array}$

$\begin{array}{ll}\text { 1.3.4 Feeding and Energy Metabolism } & 19\end{array}$

\section{Chapter 2}

Cognitive Functions Of Brain Histamine $\quad 22$

$\begin{array}{ll}2.1 \text { Recognition Memory } & 23\end{array}$

2.2 Fear Memory 23

2.3 How to evaluate memory in rodents: most widely used paradigms $\quad 24$

2.4 The role of central histaminergic system in memory and cognition 25

\section{Chapter 3}

Disorders Associated With Brain Histamine

\section{Chapter 4}

Is Histaminergic Neurotransmission Involved in Antidepressant Responses?

4.1 Historical background 30

4.2 The modern concept of depression 31

$\begin{array}{ll}4.3 \text { Neurogenic and neurotrophic theory } & 31\end{array}$

4.4 Role of CREB in Depression and Antidepressant Treatments 33

$\begin{array}{ll}4.5 \text { Neurocircuitry of depression } & 34\end{array}$

4.6 Animal Models used to screen antidepressant compounds 35

4.6.1 Tail Suspension Test (TST) 35 
Histaminergic neurotransmission as a gateway for the effects of Oleoylethanolamide

4.6.2 Forced Swim Test (FST)

4.6.3 Chronic Mild Stress (CMS) 36

4.6.4 Learned Helplessness $\quad 37$

4.6.5 Novelty-suppressed feeding and sucrose preference $\quad 37$

4.6.6 Neuronal histamine: an insight on depression 37

\section{Chapter 5}

Is Neuronal Histamine Involved In Stress-Related Responses?

$\begin{array}{ll}5.1 \text { Historical Background } & 40\end{array}$

5.2 Definition and Classification of Stress 41

5.3 Neuroanatomy and Physiology of Stress 41

5.4 Stress: social behaviour, and resilience $\quad 42$

5.5 Stress impact on memory function 43

5.6 Chronic Stress Paradigm in rodents 44

5.6.1 Chronic Social Defeat Stress 45

5.6.2 Crowding and Isolation $\quad 46$

$\begin{array}{ll}\text { 5.6.3 Social Instability } & 46\end{array}$

5.6.4 Chronic Restrain Stress 46

5.7 Neuronal histamine: an insight on stress $\quad 47$

\section{Chapter 6}

Histamine And The Gut-Brain Axis $\quad 48$

6.1 Oleoylethanolamide 49

Aim of the Study $\quad 53$

$\begin{array}{ll}\text { Results } & \mathbf{5 5}\end{array}$

\section{Part I}

Histaminergic Neurotransmission As A Gateway For The Cognitive Effect Of Oleoylethanolamide In Contextual Fear Conditioning $\mathbf{5 5}$

$\begin{array}{ll}\text { 1.1 Materials and Methods } & 55\end{array}$

$\begin{array}{ll}\text { 1.1.1 Animals and Drugs } & 55\end{array}$

$\begin{array}{ll}\text { 1.1.2 Surgery } & 55\end{array}$

1.1.3 Infusion Procedure and Experimental Groups 56

1.1.4 Contextual Fear Conditioning $\quad 56$

1.1.5 Freezing Measurement $\quad 56$

$\begin{array}{ll}1.1 .6 \text { Histology } & 57\end{array}$

$\begin{array}{ll}\text { 1.1.7 Statistical Analysis } & 57\end{array}$

$\begin{array}{ll}1.2 \text { Results } & 57\end{array}$

1.2.1 Oleoylethanolamide administration increases freezing time of rats submitted to contextual fear conditioning 57

1.2.2 Histaminergic neurotransmission is required for OEAfreezing enhancements 
1.2.3 Antagonism of histamine $\mathrm{H} 1$ and $\mathrm{H} 2$ receptors prevents

OEA-induced freezing enhancement

1.3 Summary of Results (Part I)

\section{Part II}

Oleoylethanolamide Induces Antidepressant-Related Responses By Targeting PPAR- $\alpha$ And Recruiting The Histaminergic Neurotransmission 61

2.1 Materials and Methods

2.1.1 Animals and Drugs

2.1.2 Behavioral Experiments

2.1.2.1 Tail Suspension Test (TST)

2.1.2.2 Open Field test

2.1.3 Neurochemical experiments

2.1.3.1 Western Blot analysis $\quad 62$

2.1.4 Statistical Analysis $\quad 63$

2.2 Results

2.2.1 Oleoylethanolamide systemic administration exerts antidepressantlike effect by recruiting histaminergic neurotransmission

2.2.2 OEA-induced increase in cortical and hippocampal CREB phosphorylation is reduced in HDC-KO mice

2.2.3 Oleoylethanolamide systemic administration reduced immobility time by targeting PPAR- $\alpha$

2.2.4 OEA-induced increase in cortical and hippocampal CREB phosphorylation is reduced in PPAR-alpha $\mathrm{KO}$ mice

2.3 Summary of the Results (Part II)

\section{Part III}

Histaminergic Involvement In Oleoylethanolamide Protection On The Cognitive Decline Induced By Chronic Social Stress In Mice

3.1 Materials and Methods

3.1.1 Animals and Drugs

3.1.2 Chronic Social Defeat Stress Paradigm

3.1.3 Social Interaction Test

$\begin{array}{ll}3.1 .4 \text { Novel object recognition test } & 68\end{array}$

$\begin{array}{ll}3.1 .5 \text { Statistical analysis } & 69\end{array}$

3.2 Results (Part III)

3.2.1 Chronic Social Defeat Stress induced body weight gain and increased food consumption in OEA or VEH treated mice compared to controls

3.2.2 Oleoylethanolamide reduces social avoidance induced by social defeat stress

3.2.3 Oleoylethanolamide improves the performance in the object recognition test of WT stressed mice, but not of HDC-KO mice

3.2.4 CSDS did not affect motility of mice tested in the open field 70

3.3 Summary of the Results (Part III) 
Histaminergic neurotransmission as a gateway for the effects of Oleoylethanolamide

Conclusions And Discussion

References

75

Figures 


\section{Abstract}

The first description of histamine dates back to Sir Henry Dale and co-workers (Dale HH, 1910; Kwiatkowski, 1941) who detected its presence and biological activity. Dale and colleagues by studying the effect of ergot extracts, identified histamine as stimulant of smooth muscle in the gut and respiratory tract by induction of vasodilatation. Simultaneously, at the beginning of XX century, the stimulation of acid secretion in the stomach caused by histamine was also recognized. During the first 20 years after these discoveries other important histaminergic actions were defined. Lewis and Grant (LEWIS T., 1924) headlines: Vascular reaction of the skin to injury. The liberation of a histamine-like substance in injured skin: the underlying cause of factitious urticarial and of wheals produced by burning, and observation upon the nervous control of skin reactions. With this discovery was defined for the first time what will be then call "triple response of Lewis". The rapid developments of these years soon ceased, however, and despite continuous efforts and considerable improvements in the available technical facilities the experimental data since presented to show that histamine participates in physiological processes appear to be inconclusive. The presence of histamine in the brain, predominantly in the grey matter, was first shown by Kwiatkowski(Kwiatkowski, 1941), and White (WHITE, 1959) but in 1960 's with the introduction of fluorimetric assay that disclosed the anatomical identity of the catecholamine containing neurons and their projections, histamine was initially neglected because the assay failed to determine the location of histamine in the brain. This was because the reagents that were used to detect this diamine cross-reacted with spermidine - a uniformly distributed polyamine that occurs at high concentrations (Haas and Panula, 2003). But circumstantial evidence began to accumulate for a significant role for histamine, and lesion studies by the Schwartz group, in 1974, pointed to the approximate location of a brain source of histamine (Garbarg et al., 1974). Later, two different and parallel discoveries brought to a general acceptance of the existence of central histaminergic system. Panula and colleagues and Watanabe and co-workers in 1984 independently demonstrated by means of immunohistochemistry that the tuberomamillary nucleus (TMN) in the posterior hypothalamus, is the only source of histaminergic neurons and the origin of the widely distributed histaminergic projections just like the other amine systems (Panula et al., 1984; Takeda et al., 1984). It is now clear that the histaminergic TM system commands general states of metabolism like satiety and appetite, arousal, and of consciousness, including learning and memorizing both pleasurable and aversive events.

In my thesis, I will provide evidence that in the brain histaminergic system converge peripheral and central signals and orchestrates appropriate behavioural responses. In particular, I will present results that strongly suggest the involvement of 
Histaminergic neurotransmission as a gateway for the effects of Oleoylethanolamide

histamine neurons in the pro-cognitive and antidepressant-like effects of oleoylethanolamide, a lipid that is normally secreted by the intestine after a fatty meal, and I will present data on some of the molecular mechanisms responsible for these effects. I will also illustrate the influence of dietary factors on stress reactivity and cognition and preliminary results on the role of brain histamine in such a model.

The results exposed in this thesis may have relevance in revealing new mechanisms in the gut brain axis when brain histamine plays a key role as it acts as a gatekeeper of peripheral and central signals to elaborate the appropriate behaviour. 


\section{Chapter 1 \\ Histamine in the Nervous System}

\subsection{Anatomic Framework}

In the central nervous system, the presence of histamine is attributed at the presence of the histamine-releasing neurons. The amount of histamine derived by nonneuronal pool (mast cells) is somewhat limited under normal conditions. Other possible sources of histamine in the brain may include microglia and microvascular endothelial cells (Katoh et al., 2001; Yamakami et al., 2000). Mast cells are relatively scarce in the brain, in comparison to other tissues, and their function is at present unclear. Furthermore, the amount of peripherally synthetized histamine not contribute to its central content due to the histamine inability to cross the blood brain barrier. Therefore, it can be assumed that central histaminergic function are due almost exclusively to histaminergic neurons (Brown and Ennis, 2001). As previously reported, the histamine-producing neurons are located in the small tuberomamillary nucleus (TMN). The name, tuberomamillary nucleus, derives from the anatomical term tuber cinerum, denoting an ashen swelling located rostral to the mammillary bodies and caudal to the optic chiasm, forming the floor of the third ventricle in the hypothalamus (Krüger and Nyland, 1995). The TM in rats has been subdivided by Ericson et al. (Ericson et al., 1987) into three subgroups: (I) the medial tuberomamillary subgroup (TMM), which consists of around 600 neurons located on either side of the mammillary recess; (II) the ventral tuberomamillary subgroup (TMV), which contains approximately 1500 neurons around the mamillary bodies; and (III) the diffuse part of the TM (TMdiff or E5), which is made up of about 100 HD-immunoreactive perikarya scattered within or between various hypothalamic nuclei (Inagaki et al., 1990). In the mouse brain the TMN is less compact and is characterized by smaller and fewer neuron than rat TMN (Parmentier et al., 2002). The human TMN consist of about 64.000 neurons anatomically identified as the ventral, medial area and the lateral area (Airaksinen et al., 1991a).

The histamine neurons in the TM send projections that innervate the entire brain, and parts of the spinal cord (Figure 1) (Panula et al., 1984; Watanabe et al., 1984). Two ascending pathways and one descending pathway have been identified (Panula et al., 1989). The highest density of histaminergic fibres are found in the hypothalamus, diagonal band, septum and olfactory tubercle. Moderate density of fibres are found in cerebral cortex, striatum and nucleus accumbens. Projections to the midbrain, brain stem, cerebellum and spinal cord tend to be of lower density. The hippocampal formation is most strongly innervated in the subiculum and dentate gyrus, with a low 
Histaminergic neurotransmission as a gateway for the effects of Oleoylethanolamide

density of fibres present in CA3 and CA1(Brown and Ennis, 2001). The afferent projections to TM neurons are widespread and come from many different areas. Prominent sources are the infralimbic prefrontal cortex, lateral septum and preoptic nucleus (Ericson et al., 1991). Most of the efferent histaminergic fibers are unmyelinated and except for those that project to the trigeminal nucleus (Inagaki et al., 1988), do not in general form synaptic specializations, rather, histamine is released from varicosities located periodically along the axon (Takagi et al., 1986). Thus, histamine release sites and histamine receptors are not directly associated to one another. Rather, histamine has been proposed to act like a local hormone on neurons, glial cells and blood vessels in a concerted manner (Wada et al., 1991). In addition to histamine, the TM neurons contain several other neurotransmitters and modulators like GABA that is presumably released in specific brain regions to modulate behavioural response (Williams et al., 2014; Yu et al., 2015b). The neuropeptides GALANIN, thyrotropin-releasing hormone, proenkephalin-derived peptides and substance $P$ are also found in various populations of histamine producing TM neurons. Most TM neurons also express adenosine deaminase (ADA), the enzyme that catalyzes the conversion of adenosine to inosine (Haas and Panula, 2003).

\subsection{Histaminergic Receptors}

The basic homeostatic and higher functions, including cognition, arousal, circadian and feeding rhythms regulated by brain histamine are due to the action on 4 metabotropic receptors: $\mathrm{H}_{1} \mathrm{R}, \mathrm{H}_{2} \mathrm{R}, \mathrm{H}_{3} \mathrm{R}, \mathrm{H}_{4} \mathrm{R}$. All of histaminergic receptors are expressed at central level with different density in different brain regions (Passani and Blandina, 2011). All metabotropic histamine receptors $\left(\mathrm{H}_{1} \mathrm{R}-\mathrm{H}_{4} \mathrm{R}\right)$ belong to the rhodopsin-like family of $\mathrm{G}$ protein coupled receptors (GPCR). Each receptor consists of seven large transmembrane-spanning elements with prototypic domains (Haas et al., 2008). Three of the four histamine receptors that have been identified $\left(\mathrm{H}_{1}-\mathrm{H}_{3}\right)$ are prominently expressed in the brain in specific cellular compartments, whereas the fourth $\left(\mathrm{H}_{4}\right)$ receptor is detected predominantly in bone marrow and leukocytes (Haas and Panula, 2003).

\subsubsection{Histamine $\mathrm{H}_{1}$ Receptor}

The human $\mathrm{H}_{1}$ receptor is encoded by a gene of $56 \mathrm{kDa}$ composed by $487 \sim 490$ amino acids located on chromosome 3p25 (Jongejan et al., 2005). The signal transduction of $\mathrm{H}_{1} \mathrm{R}$ is mainly mediated by coupling to $\mathrm{Gq} / 11$ proteins (Gutowski et al., 1991; Leopoldt et al., 1997; Moniri et al., 2004; Selbach et al., 1997), but also signals via Gi/o in some systems (Seifert et al., 1994; Wang and Kotlikoff, 2000), and the small $\mathrm{G}$ protein family, most likely through an indirect downstream effect (Mitchell and Mayeenuddin, 1998). The interaction of $\mathrm{H}_{1}$ receptor with $\mathrm{Gq} / 11$ protein and phospholipase $\mathrm{C}$ promotes inositol trisphosphate (IP3)-dependent $\mathrm{Ca} 2+$ release from intracellular $\mathrm{Ca}^{2+}$-stores, and also diacylglycerol formation. $\mathrm{H}_{1} \mathrm{R}$ also activates AMPkinase, nuclear factor kappa B, nitric oxide synthases, and phospholipase A2 (PLA2), which induces arachidonic acid formation (Haas et al., 2008). $\mathrm{H}_{1} \mathrm{R}$ are found throughout the whole body and nervous system. $\mathrm{H}_{1}$ receptors are widely distributed in mammalian brain (Hill, 1990; Schwartz et al., 1991). High densities are found in brain 
regions concerned with neuroendocrine, behavioural, and nutritional state control, including the periventricular, suprachiasmatic, and ventromedial nuclei of the hypothalamus, aminergic and cholinergic brainstem nuclei, thalamus, and cortex (Schwartz et al., 1991). The global loss of $\mathrm{H}_{1} \mathrm{R}$ in $\mathrm{KO}$ mice produces immunological, metabolic, and behavioural abnormalities (Haas et al., 2008; Hirai et al., 2004; Huang et al., 2006; Masaki and Yoshimatsu, 2006).

\subsubsection{Histamine $\mathrm{H}_{2}$ Receptor}

A second class of histamine receptors was identified by Black and colleagues based on the different pharmacological profile of the histamine receptor responsible for stimulating gastric acid secretion (Hill et al., 1997). The gene encoding the human $\mathrm{H}_{2} \mathrm{R}$, which is a $40-\mathrm{kDa} 359$-amino acid peptide, is located on chromosome 5q35.5. $\mathrm{H}_{2} \mathrm{R}$ couple to Gs proteins to stimulate adenylyl cyclase and increase intracellular cAMP, which activates protein kinase A (PKA) and the transcription factor CREB, all of which are key regulators of neuronal physiology and plasticity. Through $\mathrm{H}_{2} \mathrm{R}$ activation and PKA-dependent phosphorylation, histamine blocks a $\mathrm{Ca}^{2+}$-activated potassium conductance responsible for the neuronal excitability (Haas et al., 2008). Independent of either cAMP or $\left[\mathrm{Ca}^{2+}\right] \mathrm{i}$ levels, $\mathrm{H}_{2} \mathrm{R}$ also inhibit PLA2 and release of arachidonic acid, which likely account for the opposing physiological responses elicited by $\mathrm{H}_{1} \mathrm{R}$ and $\mathrm{H}_{2} \mathrm{R}$ in many tissues (Traiffort et al., 1992). Like the histamine $\mathrm{H}_{1}$ receptor, the $\mathrm{H}_{2}$ receptor has a widespread expression in the brain and spinal cord, particularly high densities are found in the basal ganglia and in parts of the limbic system such as the hippocampal formation and amygdala. In contrast to $\mathrm{H}_{1}$ receptors, $\mathrm{H}_{2}$ receptors are present in low densities in septal areas, hypothalamic and thalamic nuclei. $\mathrm{H}_{1}$ and $\mathrm{H}_{2}$ receptors are colocalized in several areas of the brain including pyramidal and granule cells in the hippocampal formation and in the other aminergic cell where the receptors can act synergistically, e.g. in the stimulation of cAMP production (Brown and Ennis, 2001). Mice deficient in $\mathrm{H}_{2} \mathrm{R}$ function exhibit selective cognitive deficits along with an impairment in hippocampal LTP (Dai et al., 2007) and with abnormalities in nociception (Mobarakeh et al., 2006; Mobarakeh et al., 2005) and gastric and immune functions (Teuscher et al., 2004).

\subsubsection{Histamine $\mathrm{H}_{3}$ Receptor}

Histamine $\mathrm{H}_{3}$ receptor in the brain were detected in 1983 by the group of J.C. Schwartz in Paris proved its neurotransmitter function as auto- as well as hetero-receptor at pre- and postsynaptic membranes and revealed its profound influence on different neurotransmitter balances (Panula et al., 2015). The gene $\left(\mathrm{Hrh}_{3}\right)$ encoding human $\mathrm{H}_{3} \mathrm{R}$, a 70-kDa 445-amino acid peptide, is located on chromosome 20q13.33. $\mathrm{H}_{3} \mathrm{R}$ negatively couple through pertussis toxin-sensitive Gi/o proteins to $\mathrm{N}$ - and $\mathrm{P}-$ type $\mathrm{Ca}^{2+}$ channels and to adenylyl cyclase. Through extensive cross-talks with other GPCRs, they can also engage Gq/11 signaling and activate PLA2, Akt/GSK3, and MAP kinase pathways, all of which play important roles in axonal and synaptic plasticity and a variety of brain disorders (Haas et al., 2008). The histamine $\mathrm{H}_{3}$ receptor is located on histaminergic neuron somata, dendrites and axon varicosities, as well as on 
Histaminergic neurotransmission as a gateway for the effects of Oleoylethanolamide

the axon varicosities and somata of other neurons, providing negative feedback to inhibit histamine synthesis and the release of histamine or other transmitters, including glutamate (Brown and Reymann, 1996; Doreulee et al., 2001), acetylcholine (Arrang et al., 2007; Passani and Blandina, 1998) and GABA (Jang et al., 2001; Yamamoto et al., 1997). In keeping with their role as auto- and hetero-receptors, $\mathrm{H}_{3} \mathrm{R}$ are heterogeneously distributed among areas known to receive histaminergic projections (Hu and Chen, 2017). High densities are found particularly in anterior parts of the cerebral cortex, hippocampus, amygdala, nucleus accumbens, striatum, olfactory tubercles, cerebellum, substantia nigra, and brain stem. In the $T M N, H_{3} \mathrm{R}$ reside on perikarya of histaminergic neurons. Loss of $\mathrm{H}_{3} \mathrm{R}$ function in $\mathrm{KO}$ mice is associated with behavioral state abnormalities, reduced locomotion (Toyota et al., 2002), a metabolic syndrome with hyperphagia, late-onset obesity, increased insulin and leptin levels (Tokita et al., 2006; Yoshimoto et al., 2006), and an increased severity of neuroinflammatory diseases (Teuscher et al., 2007).

\subsubsection{Histamine $\mathrm{H}_{4}$ Receptor}

Six independent laboratories contributed to the identification and cloning of the $\mathrm{H}_{4}$ receptor (Liu et al., 2001; Morse et al., 2001; Nakamura et al., 2000; Nguyen et al., 2001; O'Reilly et al., 2002; Oda et al., 2000; Zhu et al., 2001). The human $\mathrm{H}_{4}$ receptor gene is present on chromosome $18 \mathrm{q} 11.2$ and is a $44 \mathrm{kDa} 390$-amino-acid polypeptide. Like $\mathrm{H}_{3} \mathrm{R}$, the $\mathrm{H}_{4}$ receptor is coupled to pertussis toxin sensitive $\mathrm{Gi} / \mathrm{o}$ protein with inhibitory effect on cAMP accumulation (Leurs et al., 2009: Oda et al.. 2000). $\mathrm{H}_{4}$ receptor expression has been observed in eosinophils, $\mathrm{T}$ cells, dendritic cells, basophils, and mast cells (Gantner et al., 2002; Hofstra et al., 2003; Liu et al., 2001; O'Reilly et al., 2002), but its expression in the central nervous system remains controversial. In the human brain, expression of $\mathrm{H}_{4}$ receptor mRNA has been reported in the amygdala, cerebellum, corpus callosum, cortex frontal cortex, hippocampus, and thalamus (Strakhova et al., 2009) but, results obtained with analyses of mRNA expression does not always reflect results obtained with immunohistochemistry, therefore there is a debate about $\mathrm{H}_{4} \mathrm{R}$ in SNC that needs further research (Panula et al., 2015).

\subsection{Homeostatic Histaminergic Functions}

The morphology of brain histaminergic system with a compact group of cells and a capillary distribution of varicose fibres suggest its action as a normative centre for the brain activity. Pharmacological studies in intact and histamine-deficient animals as well as humans link brain histamine with homoeostatic brain functions and neuroendocrine control. Brain histamine controls behavioural responses, biological rhythms, body weight, energy metabolism, thermoregulation, fluid balance, stress, and reproduction (Hough, 1988; Parmentier et al., 2002; Schwartz et al., 1991).

Recently, our laboratory demonstrated functional differences in TMN neurons, suggesting that histaminergic neurons are organized in distinct subpopulation impinging on different brain regions (Blandina et al., 2012; Giannoni et al., 2009). 


\subsubsection{Sleep and Wakefulness}

Histaminergic neurons help sustain wakefulness, several studies corroborate this hypothesis; in $\mathrm{H}_{1} \mathrm{R}-\mathrm{KO}$ mice the sleep-wake cycle is impaired and the waking promotion induced by $\mathrm{H}_{3} \mathrm{R}$ antagonist is abolished (Huang et al., 2006; Lin et al., 2002). During waking c-fos expression increases in TMN neurons (Lin, 2000; Nelson et al., 2002; Nelson et al., 2003; Scammell et al., 2000; Sherin et al., 1998; Vanni-Mercier et al., 2003). The regulation of the transition between wakefulness and sleep involves antagonist influences of sleep-promoting VLPO neurons, which provide inhibitory GABA- and galanin-mediated inputs to TMN and brainstem cholinergic and monoaminergic groups, and excitatory effects of orexin (Hcrt/Orx) neurons on TMN and other wake-active neuronal groups (Benarroch, 2010). TMN neurons become active just after waking and fire at an average rate of about $5 \mathrm{~Hz}$, and their activity is suppressed during sleep (Sakai et al., 2010; Saper et al., 2010; Takahashi et al., 2006). A recent elegant work by Wisden and co-workers demonstrated that zolpidem, a $\mathrm{GABA}_{\mathrm{A}}$ receptor-positive modulator, needs to work on specific cell types of the brain, including histaminergic neurons, to induce sleep, without reducing the power of the sleep, hence improving sleep quality (Uygun et al., 2016). Furthermore, the same laboratory showed that wake-active histaminergic neurons generate a paracrine GABAergic signal that serves to provide a brake on over-activation from histamine, but could also increase the precision of neocortical processing (Yu et al., 2015b).

\subsubsection{Thermoregulation}

The key anatomical sites that control thermoregulation are under histaminergic innervation. The control centres of thermoregulation are located in the anterior preoptic area (thermoregulator rostral center) and in posterior hypothalamic area (thermoregulator caudal center) (Nieuwenhuys et al., 2008). Central administration of histamine in freely moving animals causes hypothermia or biphasic responses, hypofollowed by hyperthermia (Clark et al., 1975; Clark and Cumby, 1976; Cote and Harrington, 1993). Hyperthermia, in turn, facilitates neuronal histamine release (Kanamaru et al., 2001). Activation of H1Rs and H2Rs in the anterior hypothalamus/preoptic area and posterior hypothalamus respectively induced hypothermia (Benarroch, 2010; Clark and Cumby, 1976). By H1Rs and H2Rs agonist actions, histamine regulates body temperature and clock neurons, suggesting an evolutionary conserved link between histamine, circadian rhythms, and temperature control.

\subsubsection{Fluid Balance}

Histamine elicits drinking following injection into the cerebral ventricles or into several hypothalamic sites (Gerald et al., 1972; Leibowitz, 1973). In addition, histamine increases the release of vasopressin and decreases urine output via both $\mathrm{H}_{1}$ and $\mathrm{H}_{2}$ receptors (Bennett and Pert, 1974; Bhargava et al., 1973; Kjaer et al., 1994). Histamine also stimulates vasopressin release indirectly via a local release of noradrenaline (Bealer, 1993; Bealer and Abell, 1995). A physiological role for central histamine in the control of fluid balance is suggested by the findings that 24 or $48 \mathrm{~h}$ of dehydration increases synthesis and release of histamine in the hypothalamus (Kjaer et al., 1994; Kjaer et al., 1995). Furthermore, blockade of histamine synthesis by $\alpha-F M H$, 
Histaminergic neurotransmission as a gateway for the effects of Oleoylethanolamide

activation of presynaptic $\mathrm{H}_{3}$ autoreceptors or antagonism of postsynaptic histamine receptors all strongly depress dehydration-induced vasopressin release (Kjaer et al., 1994). Dehydration induced renin release also appears to involve central histamine activation of the sympathetic nervous system (Kjaer et al., 1998; Matzen et al., 1990).

\subsubsection{Feeding and Energy Metabolism}

The evidence for histamine involvement in food intake is nowadays very consistent (Provensi et al., 2016; Provensi et al., 2014). Early studies demonstrated that i.c.v injection of histamine, loading with histamine precursor L-histidine or application of the $\mathrm{H}_{3}$ receptor antagonist thioperamide suppress feeding (Cohn et al., 1973; Machidori et al., 1992; Ookuma et al., 1993; Sheiner et al., 1985), whereas i.c.v infusions of a-FMH or $\mathrm{H} 1$ receptors antagonists increase food intake (Fukagawa et al., 1989; Ookuma et al., 1989; Sakata et al., 1988a; Sakata et al., 1988b). However, the role of histamine is not restricted to feeding control but also the regulation of body weight and adiposity by modulation of peripheral energy. Many of the central hypothalamic areas involved in regulating feeding, including the arcuate, ventromedial $(\mathrm{VMH})$ and paraventricular (PVN) nucleus and lateral hypothalamic perifornical area (LHA), are densely innervated by histamine containing fibres and show a high density of $\mathrm{H}_{1} \mathrm{Rs}$ (Panula et al., 1989). Early work suggested that histamine-mediated suppression of food intake was controlled by the $\mathrm{VMH}$ as microinfusion of $\mathrm{H}_{1} \mathrm{R}$ antagonists into the VMH but not PVN or LH elicited feeding responses and increases both meal size and duration (Fukagawa et al., 1989; Sakata et al., 2003). Likewise, electrophoretic application of $\mathrm{H}_{1} \mathrm{R}$ antagonists suppressed the firing of glucose-responsive units in the VMH but not in the LHA or PVN (Fukagawa et al., 1989). Another site of importance in the histamine control of food intake is the mesencephalic trigeminal nucleus. Bilateral injections of a-FMH into this region reduced eating speed and prolonged meal duration while leaving meal size unaltered. Feeding induced increases in histamine turnover in both the trigeminal nucleus, which controls mastication, and the ventromedial area, which is considered as a satiety center (Fujise et al., 1998). In our laboratory, we recently showed that the PVN as well takes part into the histaminergic control feeding behaviour as histamine released in the PVN activates oxytocin neurons (Provensi et al., 2014) that in turn exert hypophagic behaviour (Gaetani et al., 2010). Furthermore, the orexigenic actions of orexins/hypocretins (Jørgensen et al., 2005) and the anorexigenic effects of leptin (Toftegaard et al., 2003) and glucagonlike peptide-1 (GLP-1), which depend on CRH released by PVN neurons (Gotoh et al., 2005), are all blunted or absent by pharmacological or genetic loss of $\mathrm{H}_{1} \mathrm{R}$ function. Ghrelin, another peptide of peripheral origin, does not affect histamine release, suggesting that ghrelin may act on a parallel, different mechanism that controls food intake (Ishizuka et al., 2006).

Most experimental observations in rodents agree that blockade of brain $\mathrm{H}_{3}$ receptor, hence increasing histamine release, decreases energy intake, body weight and plasma triglycerides (Hancock and Brune, 2005). Also, they increase histamine release from the hypothalamus, they reduce energy intake in normal and leptin-resistant mice with diet induced obesity (Ishizuka et al., 2008), and decrease food intake in wild type mice (Provensi et al., 2014). From the pharmacological point of view the importance of histamine in the regulation of feeding behaviour came from the observation that increased weight is a common adverse effect of many classic antipsychotic 


\section{Alessia Costa}

drugs and atypical antipsychotics that depends on their affinity as antagonists at the $\mathrm{H}_{1} \mathrm{Rs}$ (Kroeze et al., 2003). Preclinical studies showed that activation of histamine neurons induces the arousal state during food anticipation (Angeles-Castellanos et al., 2004; Inzunza et al., 2000), and during the appetitive phase that precede food consumption (Passani and Blandina, 2011). 



\section{Chapter 2 \\ Cognitive functions of brain histamine}

The regulation of memory consolidation by histamine is a topic of much recent research and a new role for the brain histaminergic system is emerging, as a gateway between peripheral signals and the elaboration of both aversive and emotionally neutral memories (Passani et al., 2017).

Memory is not a unitary faculty of the mind but is a complex process composed and coordinated by multiple systems, distinct brain regions and pathways involves. Two memory systems have been described: declarative and procedural or 'habit' memory system (Izquierdo et al., 2006b). Declarative memory ("knowing that") refers to conscious memory for events and facts, is assessed by explicit tests of recall and recognition, and depends upon medial temporal lobe and diencephalic brain structures. Procedural memory ("knowing how") refers to unconscious memory, is assessed by experience-dependent learning of skilled performance, and depends on structures in the basal ganglia, cerebellum, and neocortex (Gabrieli, 1998). The memory systems of the mammalian brain operate independently and in parallel to support behaviour (McDonald and Hong, 2013; Packard and Goodman, 2013; Poldrack and Packard, 2003).

Procedural or working memory (WM) was considered as overlapping with immediate memory or short-term memory (STM). STM was assumed to be essential for acquisition and perhaps subsequent long-term memory (LTM) formation.

It is generally accepted that the STM, that lasts minutes or few hours, may be converted in LTM that persists for days, weeks or more, by a specific sequence of events called consolidation which starts immediately after the acquisition phase (Izquierdo et al., 1999; McGaugh, 2000). Not all information reaching the CNS is stored; most inputs are filtered away by the attentional and emotional processes after the acquisition phase (Cahill and McGaugh, 1998). Only memories relevant for cognition, emotionally salient, or derived from strong sensory inputs are consolidated in LTMs. Forgetting mean entails screening, since only memories previously selected persist, are stored and retrieved by chains of cause and effect controlled by a range of factors that operate simultaneously at many different levels often implicit, but sometimes explicit. The brain systems responsible for learning and storing memories have a mechanism for preventing information overload (Izquierdo et al., 2006b). Memory can be modulated by experiences occurring about the time when it is learned, consolidated or retrieved (Cahill and McGaugh, 1998; Kandel and Squire, 2000). 
Histaminergic neurotransmission as a gateway for the effects of Oleoylethanolamide

\subsection{Recognition Memory}

Recognition memory involves at least two separable processes, familiarity discrimination and recollection and more complex aspects of contextual, associative and spatial aspects (Brown and Banks, 2015). Types of stimuli, single or multi-item, associative or non-associative stimuli, and types of information being stored, for example, the 'what', 'when', and 'where' of episodic-like memories or the modality of the sensory input allows the distinctions between types of memory process.

Discrimination for visual stimuli seems to be affected by a system centred on the perirhinal cortex of the temporal lobe (Brown and Banks, 2015). Regions other than perirhinal cortex may also be involved in recognition memory processes: when a recognition memory task is solved using recollection or association concerning a presented stimulus, the recognition memory predicts hippocampal involvement (Brown et al., 2010). The hippocampus is involved in recognition memory using multiple items and associative or spatial information (Aggleton and Brown, 2006; Dere et al., 2006; Eichenbaum et al., 2007; Murray and Ranganath, 2007; Squire et al., 2007; Winters et al., 2008). In particular, lesions of the rat hippocampus impair recognition memory that requires spatial information; location tasks are impaired by hippocampal lesions while perirhinal lesions have no effect (Warburton and Brown, 2010).

Functional imaging in human subjects has implicated the prefrontal cortex in recognition memory processes (O'Neil et al., 2012). The role of the rodent medial prefrontal cortex in recognition memory has been extensively studied. Large lesions of the prefrontal cortex, which included the anterior cingulate, prelimbic and infralimbic cortices, or which centred on the ventral medial prefrontal cortex, produced recognition impairments (Kolb et al., 1994; Ragozzino et al., 2002). The medial prefrontal cortex has been implicated in attentional processing (Chudasama and Robbins, 2003; Muir, 1996), and play an important role in temporal order memory (Chiba et al., 1997; Hannesson et al., 2004; Mitchell and Laiacona, 1998). Lesions in the medial prefrontal cortex impaired temporal order memory task (Barker et al., 2007; Devito and Eichenbaum, 2011) but not induces deficits in the recognition or location tasks (Barker et al., 2007). Moreover, in humans and non-human primates damage to the medial dorsal thalamus (MD nucleus) produces recognition memory deficits (Parker et al., 1997; Victor, 1987; Warburton and Brown, 2015).

\subsection{Fear Memory}

Forming associations about events and then consolidating memories of those associations is an important strategy for survival. However, in traumatic situations, these associations sometimes become overly consolidated and then, potentially, are resistant to extinction over time, resulting in fear-related disorders (Parsons and Ressler, 2013). But fear, in general, has a strong survival value. The lack of fear, also called recklessness or mindlessness in humans, is inherently dangerous and potentially lethal (Izquierdo et al., 2016). Therefore, in both cases, over-consolidation/resistant-extinction and recklessness/loss of fear are two side of the same coin, dangerous in the same way.

The acquisition and memory of conditioned fear depend on both hippocampus and amygdala, as lesion studies (Lorenzini et al., 1996a; Lorenzini et al., 1996b; Sacchetti et al., 1999; Sacchetti et al., 2002) and biochemical studies (Trifilieff et al., 
2007) indicate. The sensory-related information from hippocampus and amygdala presumably originates in the mesencephalic reticular formation and ventral tegmental area (VTA), which receive it, in turn, from collaterals of the sensory pathways (BUSER and ROUGEUL, 1961; GREEN and MACHNE, 1955; MACHNE and SEGUNDO, 1956). The hippocampus and the basolateral amygdala (BLA) together with the ventro-medial prefrontal cortex (vmPFC) orchestrate memory formation (Izquierdo et al., 2016). Also the periaqueductal grey (PAG), is a brain region that conveys aversive signals to the amygdala. The PAG is known as an output structure for various conditioned fear responses, it receives a strong nociceptive input from the spinal and trigeminal dorsal horn (Gross and Canteras, 2012) and a recent study found that temporary pharmacological inactivation of PAG reduces shock-evoked responding in amygdala neurons and the acquisition of fear learning (Johansen et al., 2011).

\subsection{How to evaluate memory in rodents: most widely used paradigms}

Fear learning is usually studied by classical (Pavlovian) or instrumental association between the environment or changes in the environment (conditioned stimulus, CS) and a fearsome stimulus (usually one or more mild foot shocks; unconditioned stimulus, US). This type of learning represents situations in humans in which initially neutral stimuli become threatening through pairing with other stimuli and generate fear, a human emotion that guides much of our behavior and is crucial for survival (Izquierdo et al., 2016).

In the fear conditioning paradigm, the animals are placed in a new environment (context) were they receive a mild aversive stimulus such as a foot shock (US) associated with another stimulus such as tone or light (CS), that usually does not elicit a response. Following learning, the presentation of the CS alone generates various visceral and behavioural conditioned fear responses. The term fear response is used to refer specifically to measurable responses that occur in response to threat and not to the conscious feelings of fear: called freezing behaviour. Freezing behaviour (conditioned response, CR), is a generalized immobility caused by a generalized tonic response of the animals' skeletal musculature except those muscles used in breathing (Herry and Johansen, 2014; Izquierdo et al., 2016).

Promnesic agents are expected to increase, whereas amnesic manipulations to reduce freezing behaviour (Curzon et al., 2009; Wehner and Radcliffe, 2004).

The most widely used instrumental fear conditioning is one-trial inhibitory avoidance (IA) (Gold, 1986; Izquierdo et al., 2006a; Izquierdo and Medina, 1997) which used to be called "passive avoidance" in opposition to the "active avoidance" tasks in which animals have to perform some movement to avoid the foot shocks. In the "passive" tasks animals have to withhold stepping through a hole into a dark compartment, or stepping down from a platform onto a grid, to access the shock compartment; the required response is to remain in the safe, lit compartment or on the start platform. Animals learn to avoid stepping through or stepping down, but they are not in any way refrained from moving or behave in any way passively. In fact, they move a lot while on the platform or in the lit compartment (Netto and Izquierdo, 1985). When retested, an increase in the latency to step-through or to step-down is related as a measure of learning (Izquierdo and McGaugh, 2000).

During the last decade the 'what', 'where' and 'when' (WWWhen) episodic-like memory (ELM) task, which is based on the object recognition paradigm, has been 
Histaminergic neurotransmission as a gateway for the effects of Oleoylethanolamide

utilized for the cognitive phenotyping of mouse mutants and transgenic mouse models of neuropsychiatric diseases. It was also widely used to identify the neuroanatomical, electrophysiological and pharmacological foundations of ELM formation, retention and retrieval (Binder et al., 2015). The object recognition paradigm is one-trial task, does not involve learning of rules, does not require reinforces and is purely based on the innate preference of the rodents to explore the novel object. Thus, a rodent that remembers the familiar object will spend more time exploring a novel object rather than the familiar one (Leger et al., 2013). The preference for the novel object is the result of incidental learning occurring during the sample phase and exploratory behaviour during the testing phase (Jablonski et al., 2013).

\subsection{The role of central histaminergic system in memory and cognition}

Recent findings have reasserted the role of histamine in the regulation of memory consolidation first proposed in 1986 in an inhibitory avoidance task in rats (Passani et al., 2017). The first description on the role of histamine in memory process came from de Almeida and Izquierdo, 1986 (de Almeida and Izquierdo, 1986); the authors demonstrated that the infusion of histamine in the lateral ventricles facilitates fear memory process when tested in the inhibitory avoidance paradigm. At that time, though, it was not entirely clear, however, whether histamine had a physiological role in memory modulation. We now know that histamine exerts different effects in different brain regions and different modulatory actions depending on the type of memory task and the phase of memorization (Passani et al., 2017; Passani et al., 2007). In the hippocampus and amygdala the histaminergic neurotransmission exerts different actions. Histamine or $\mathrm{H}_{3}$ antagonists infusion in the hippocampus ameliorated the performances in spatial learning (Huang et al., 2003). Histamine infusion in the dorsal hippocampus facilitates fear memory by activating $\mathrm{H}_{2}$ receptor (da Silva et al., 2006), whereas in the ventral hippocampus $\mathrm{H}_{1}$ receptor inhibits fear memory (Alvarez and Banzan, 2008).

When histamine was locally injected into dorsal and ventral regions of the hippocampus improvements in the rats performances in the inhibitory avoidance test (Alvarez and Banzan, 1996, 2001, 2008; da Silva et al., 2006) and also in the radialmaze tasks (Huang et al., 2003; Yamamoto et al., 2007) were observed. Conversely, blockade of endogenous histamine production by $\alpha-\mathrm{FMH}$ infusion into the ventricles inhibits the consolidation of IA, which is known to be sustained by both the BLA and the hippocampus (Izquierdo et al., 2006a; Izquierdo et al., 1992; Izquierdo and Medina, 1997; McGaugh, 2000, 2015).

The $\mathrm{H} 1$ and $\mathrm{H} 2$ histamine receptors in BLA, hippocampus, and vmPFC facilitate memory consolidation in the inhibitory avoidance task (IA) and contextual fear conditioning (CFC), and their specific antagonists have an opposite effect in the consolidation of different tasks (Benetti et al., 2012; Benetti and Izquierdo, 2013; Fiorenza et al., 2012).

A recent, seminal observation by Benetti et al. (2015) (Benetti et al., 2015) revealed that histaminergic neurotransmission provides the brain with the plasticity necessary to ensure memorization of emotionally salient events, through recruitment of alternative circuits. The authors found that the integrity of the brain histaminergic system is necessary for long-term, but not for short-term memory of step-down inhibitory avoidance. In addition, they observed that phosphorylation of cyclic adenosine 


\section{Alessia Costa}

monophosphate responsive-element-binding protein (CREB), a crucial mediator in long-term memory formation (Bernabeu et al., 1997; Josselyn et al., 2004) correlates anatomically and temporally with histamine-induced memory retrieval, showing the active involvement of histamine in the CA1 region of the hippocampus and in the basolateral amygdala in different phases of memory consolidation. 



\section{Chapter 3 \\ Disorders associated with brain histamine}

No disease entity has so far been linked specifically or selectively to brain histamine dysfunction, but histamine dysfunction may thus be a precipitating factor for epigenetic disease susceptibility, severity, and progression.

Histamine as the major wake-promoting neurotransmitter in the CNS and plays a role in the pathogenesis of sleep disorders. Cerebrospinal fluid histamine levels are decreased in people with idiopathic hypersomnia or narcolepsy, supporting the concept that histamine in humans promotes alertness and wakefulness (Kanbayashi et al., 2009; Nishino et al., 2009). Narcolepsy is a sleep disorder characterized by excessive daytime sleepiness, catalepsy and narcoleptic episodes. Insomnia is usually treated with benzodiazepines, whereas narcolepsy was treated until recently with wakefulness-promoting compounds, like modafinil, and amphetamines that modulate the dopaminergic system. In 2015, the European Medicines Agency (EMA) has recommended pitolisant, a first-in-class medicine that acts on histamine $\mathrm{H} 3$ receptors in the brain that blocks autoreceptors, for the treatment of narcolepsy with or without cataplexy.

In contrast to other aminergic systems, both histamine and the levels of its metabolites increase in the spinal fluid with increasing age (Prell et al., 1988). In Alzheimer's disease, several subcortical ascending projections, including the histaminergic neurons, display degeneration and tangle formation (Swaab et al., 1998). In the hypothalamus, neurofibrillary tangles occur exclusively in the TM nucleus (Airaksinen et al., 1991b) and histamine levels is reduced in different brain areas including hippocampus, hypothalamus, frontal and temporal cortex (Panula et al., 1998; Shan et al., 2012) suggesting that histaminergic neurons undergo degeneration and contribute to cognitive decline in this disorder. $\mathrm{H}_{3} \mathrm{R}$ antagonists improve cognitive performance in experimental animals. So far, though, clinical trials with these compounds have proven unsuccessful (NIH website). The TM neurons seem morphologically normal in patients with Parkinson's disease (PD) (Nakamura et al., 1996), and normal histidine decarboxylase activity has been observed (Garbarg et al., 1983) . In contrast, histamine levels are markedly increased in the substantia nigra, putamen and globus pallidus (Rinne et al., 2002), but only modest changes in t-MeHA, the main metabolite of histamine, and histamine-N-methyltransferase activity (Fogel et al., 1994) indicating that the increased histamine might not be present in the releasable pool in the brains of these patients. This might be due to the limited capacity of the vesicular monoamine transporter VMAT-2 to take up histamine into the vesicle. 
Histaminergic neurotransmission as a gateway for the effects of Oleoylethanolamide

Thus, the alteration in histamine may be a direct consequence of dopamine deficiency, a hallmark of Parkinson's disease. The abnormalities in the histaminergic system is highlighted in PD patients which often experienced fragmented sleep and insomnia. Alteration in the histaminergic system is also revealed in receptor binding profile. $\mathrm{H}_{3} \mathrm{R}$ binding is abnormally high in the Parkinsonian substantia nigra (Anichtchik et al., 2000), and the same phenomenon is seen in rats after depletion of nigrostriatal dopamine stores using 6-OHDA (Ryu et al., 1994). However, $\mathrm{H}_{3} \mathrm{R}$ antagonists improve motor coordination in neonatally 6-OHDA-lesioned rats, whereas imetit, an $\mathrm{H}_{3} \mathrm{R}$ agonist, attenuates 3,4-dihydroxyphenylalanine (1-DOPA)-induced increases in striatal extracellular dopamine and dyskinesia (Nowak et al., 2008). Histamine, via activation of $\mathrm{H}_{3} \mathrm{Rs}$, thus regulates the cortico-striato-nigral system at all levels (Panula and Nuutinen, 2013).

The first antipsychotics were developed as antihistaminic compounds, before dopamine was recognized as a key neurotransmitter in the brain. It is now known that the atypical antipsychotic drugs have an affinity for many different receptors, including those for serotonin, noradrenaline and histamine. In the brains of people with schizophrenia, various changes are found in the histaminergic system (Jin et al., 2009; Prell et al., 1995). Levels of t-MeHA are elevated in the spinal fluid of patients with schizophrenia (Prell et al., 1995), this implies that there is increased histamine release and turnover that could explain the low $\mathrm{H}_{1}$-receptor binding that is observed in the frontal cortex of people with schizophrenia (Nakai et al., 1991). Histamine- $\mathrm{H}_{3} \mathrm{R}$ binding is increased in the dorsolateral prefrontal cortex but unchanged in the temporal cortex of patients with schizophrenia compared with those brain regions in healthy control subjects (Jin et al., 2009). These differences may reflect the structural abnormalities of the cortical network and changes in cellular composition that underlie the functional impairments in this disorder. All antipsychotics act on dopamine $\mathrm{D}_{2} \mathrm{R}$, supporting the proposition of dopaminergic supersensitivity as a major factor in disease susceptibility and pathogenesis (Seeman et al., 2006). $\mathrm{H}_{3} \mathrm{R}$ antagonist/inverse agonist increase the dopamine and acetylcholine levels in the cortex but not in striatum, suggesting that negative symptoms and cognitive deficits could be ameliorated. Preclinical and clinical studies indicate that $\mathrm{H}_{3} \mathrm{R}$ antagonist/inverse agonist are not sufficient as antipsychotic therapy per se but represent a promising poly-therapy treatments in schizophrenics. Thus, antagonist/inverse agonist $\mathrm{H}_{3} \mathrm{R}, \mathrm{D}_{2} \mathrm{R}, 5-\mathrm{HT}_{2} \mathrm{~A}$ combination, could be considered a rational approach in different types of psychosis treatments (Ito, 2009; Tiligada et al., 2009; von Coburg et al., 2009). 


\section{Chapter 4 \\ Is histaminergic neurotransmission involved in antidepressant responses?}

\subsection{Historical background}

The term depression began to appear in the 19th century as did the modern concept of affective disorders, with the core disturbance now viewed as one of mood. Melancholia was recognized as early as the time of Hippocrates and continued through galenic medicine and medieval times. The earlier connotation of the term was very wide and included all forms of quiet insanity. Melancholia later became more clearly associated with the more modern idea of melancholy or despair, for instance, in the classic work of the English Renaissance author, Richard Burton, The Anatomy of Melancholy, first published in 1621.

The first division of the major endogenous psychoses is related to Kraepelinian dichotomy that delineates the foundations of the modern classification of psychiatric disorders. This division was formally introduced in the sixth edition of Emil Kraepelin's psychiatric textbook Psychiatrie. Ein Lehrbuch für Studirende und Aertze, published in 1899 (Decker, 2007). Kraepelin regarded psychiatric disorders as disease entities based on a medical, neurological model, with specific, organic etiology and pathology. He did, however, regard some pathological depressions as psychogenic in origin. While he did not completely clarify his views on their position in his classification, or how they were to be distinguished from manic-depressive illness with incidental stress, he appeared to regard them as a separate, but relatively small and unimportant group.

Another school of European psychiatrists was developing a very different approach. The psychoanalysts. Freud and Abraham developed a theory of the origin of depression in relation to actual or symbolic losses of a love object. Here was a theory regarding the origin of most, if not all, depressions as psychogenic. Psychological theories of causation became more widely accepted for these disorders. A challenge now arose as to how to reconcile these theories with older ones of organic causation. Adolf Meyer, a Swiss psychiatrist moved away from the idea of clear cut disease entities, and viewed all psychiatric disorders as reaction types, or psychobiological reactions of the organism to stress (Meyer, 1922). The Meyerian concept defined depression as a type of reaction in which both psychological and organic factors had to be taken into account (Paykel, 2008). 
Histaminergic neurotransmission as a gateway for the effects of Oleoylethanolamide

\subsection{The modern concept of depression}

Depression is among the leading contributors to the global burden of disease (Whiteford et al., 2013), globally, more than 300 million people of all ages suffer from depression. It is associated with enormous personal suffering and societal economic burden (Kessler et al., 2003) with 10-30\% of women and 7-15\% of men likely to suffer from depression in their life-time (Briley and Moret, 2000). Furthermore, depression can be a lethal illness owing to an elevated risk for suicide (Trivedi et al., 2006), as well as increased risks of cardiac disease, cerebrovascular disorders and other medical causes of mortality (Walker et al., 2015). The magnitude of the clinical burden of depression reflects, in part, the limited effectiveness of present-day treatments. Currently available antidepressant medications, alone and in combination, are associated with high rates of partial responsiveness or non-responsiveness, delayed response onset of weeks to months and limited duration of efficacy (Gaynes et al., 2012). Despite the high prevalence of depression and its socioeconomic impact, the etiology and pathophysiology of this complex disorder is not well understood. It is the lack of understanding of the underpinning of depression that has resulted in no substantial improvement to antidepressant treatment (Misra, 2012) and consequently the lack of improvement over the conventional monoaminergic-based therapies discovered by serendipity decades ago (Covington et al., 2010; Kessler et al., 2008).

A major liability of the monoamine-deficiency hypothesis is its derivation from the mechanism of currently available antidepressants. Despite their efficacy, however, current antidepressant pharmacotherapy alleviates symptoms in approximately two thirds of patients proving a clinical response to these agents, whereas one third have a response to placebo (Mann, 2005). Perhaps the mechanism of depression is not related to monoamines in two of three cases (Belmaker and Agam, 2008). Indeed, the monoaminergic theory for depression is not able to explain the fact that biochemical effects of antidepressant rapidly occur whereas the therapeutic response has a delay of days or weeks (Stahl, 2008). Thus, the immediate effects of antidepressant drugs on monoamines cannot fully explain this lag period of treatment response (Malberg and Blendy, 2005).

To better understand mechanisms underlying the therapeutic efficacy of antidepressant drugs, research efforts have focused on the long-term molecular changes the underlie depression and antidepressant treatments.

\subsection{Neurogenic and neurotrophic theory}

The supposition that monoamine deficits may not reflect a core feature of depression pathophysiology, but are the result of neural dysfunction directed research away from monoamines and towards the putative role of growth factors such as brain-derived neurotrophic factor (BDNF), known to be critically involved in regulating neural structure and plasticity in the adult brain (Kafitz et al., 1999; Thoenen, 1995). The first studies to implicate BDNF in antidepressant responses showed that conventional antidepressant drugs, as well as electroconvulsive therapy (ECT), enhanced BDNF and TrkB mRNA expression in the hippocampus and cortical regions in a timeframe similar to the onset of antidepressant-like response (Nibuya et al., 1995; Nibuya et al., 1996). To more directly examine the causal involvement of BDNF in antidepressant 
responses, Siuciak and colleagues infused BDNF protein directly into the midbrain and observed an antidepressant-like effect in rodents (Siuciak et al., 1997).

In humans, acute and chronic stress both decrease endogenous neurotrophin levels and can lead to significant atrophy of the hippocampus, a structure known to be involved in controlling emotionality (Duman, 2004; McEwen, 2000; Sapolsky, 1996).These events may be causally linked via neurogenesis (Duman, 2005).

Neurotrophins are growth factors with crucial roles in the formation and plasticity of neuronal networks (Huang and Reichardt, 2003). The neurotrophin family include nerve growth factor (NGF), BDNF, neurotrophin-3 (NT-3) and neurotrophin-4 (NT4). They are initially synthesized as precursor proteins (proneurotrophins), which are processed extracellular or intracellularly to be secreted mostly in a mature, biologically active form (Matsumoto et al., 2008; Yang et al., 2009). The neurotrophins, show binding specificity for particular tyrosine kinase receptors (Trk), and non-specificity for the $\mathrm{p} 75$ neurotrophin receptor (p75NTR). In particular, pro-BDNF preferentially activates p75NTR receptor to mediate programmed neuronal death (Frade et al., 1997), to decrease dendrite complexity and spine density in hippocampal neurons (Zagrebelsky et al., 2005), and to induce long-term depression of synaptic transmission (Rösch et al., 2005; Woo et al., 2005). On the contrary, mature BDNF (mBDNF) selectively activates TrkB, a member of the tyrosine kinase receptors family, to promote survival and differentiation, increasing the branching of axons and dendrites and stabilizing synaptic contacts (Lee et al., 2001). Thus, due to the essential role of BDNF for cell differentiation, nerve growth and neuronal survival it has been implicated in several brain diseases, including depression considering its well establishes relation with low levels of BDNF expression (Neto et al., 2011).

Birth of new neurons or neurogenesis continues to occur in selected neurogenic zones in the adult brain. This includes the subventricular zone that gives rise to olfactory bulb neurons, and the subgranular zone that generates granule cells of the hippocampal dentate gyrus. Similar to regulation of BDNF in the dentate gyrus, stress and antidepressant treatments exert opposing effects on neurogenesis in the adult hippocampus. Stress and depression decrease the expression and function of BDNF in the PFC and hippocampus, structures that are implicated in depression, as well as decrease the BDNF levels in the blood of subjects with depression (Bocchio-Chiavetto et al., 2010; Krishnan and Nestler, 2008; Turner et al., 2006). Reduced neurotrophic or growth factor levels may be particularly relevant to the structural alterations caused by stress and depression, as these factors (particularly BDNF) are required for activity-dependent formation and maintenance of synaptic connections (Holtmaat and Svoboda, 2009; Jourdi et al., 2009). Studies of a human BDNF polymorphism (BDNFVal66Met) that is found in approximately $25 \%$ of the population have been insightful. The presence of the BDNFVal66Met allele, whose product results blocks the processing and release of mature BDNF, is sufficient to cause atrophy of neurons in the hippocampus (Chen et al., 2006) and medial PFC (mPFC) of mice with this allele (Liu et al., 2012). Heterozygous deletion of BDNF also decreases spine density and dendrite length of hippocampal and PFC neurons, decreases hippocampal volume and occludes the effects of chronic stress (Liu et al., 2012; Magariños et al., 2011). These findings suggest that stress could cause neuronal atrophy via inhibition of BDNF or that BDNF is required for neuronal remodelling (Duman et al., 2016).

A different point of view actually discusses the balance between neuronal death and neurogenesis as an etiological cause of this pathology (Jacobs et al., 2000; 
Histaminergic neurotransmission as a gateway for the effects of Oleoylethanolamide

Malberg et al., 2000). In fact, neurogenesis is a possible phenomenon in adults (Duman et al., 2001; Gould and Tanapat, 1999) and neurotrophins are important for nervous cells formation and differentiation (Fariñas et al., 2001; Fritzsch, 2003). Several studies have been proposed to test some of these theories relating the balance between neuronal death and neurogenesis, but most of them still lack experimental verification (Merighi et al., 2008).

\subsection{Role of CREB in Depression and Antidepressant Treatments}

The neurotrophic theory of depression led to a novel therapeutic approach in the management of depression beyond manipulation of neurotransmitter-receptor interaction, consisting in targeting signal transduction and gene expression pathways. The most investigated pathway, in that sense is the Cyclic AMP (cAMP) second messenger system, which ultimately influence gene expression by activating the transcription factor cAMP response element binding protein (CREB).

CREB is a nuclear protein. It belongs to the family of leucine zipper transcription factors that are expressed in a variety of tissues and serve diverse functions. Phosphorylation of a serine residue (S133) in its kinase inducible domain is critical to mediate its effect, as this permits recruitment of co-activator proteins and initiation of transcription (Nair and Vaidya, 2006). Activation of CREB can be accomplished by phosphorylation via cAMP-protein kinase A (PKA) pathway. PKA cascade also serves as a target for antidepressant treatment (Figure 3) (Tardito et al., 2006). CREB is regulated by diverse signalling pathways and integrates the action of numerous external stimuli, including antidepressant. A downstream consequence of enhanced CREB function is thought to be the increased expression of target genes like the neurotrophin, brain-derived neurotrophic factor (BDNF) (Figure 3), and neuropeptide $Y$ (NPY), which may contribute to the antidepressant treatment mediated changes in structural plasticity and behaviour (Conti et al., 2002; Pandey, 2003). As previously mentioned, the hippocampus is a key limbic region whose structure and function are compromised in mood disorders. Hippocampal over-expression of CREB and BDNF can mimic both the structural consequences of sustained antidepressant treatment as well as exerting antidepressant like behaviour (Chen et al., 2001). Elevated CREB$\mathrm{BDNF}$, through their protective influences on vulnerable hippocampal neurons and their ability to directly promote structural recognition, could result in repair of damaged region due to depression (Misra, 2012). Elevated CREB function can either reduce or produce depressive-like behaviour in laboratory animals. For instance, elevated CREB activity in the NAc produces various depressive-like effects in rodents (Barrot et al., 2002). Until recently, the NAC has not been considered a likely site for the pathophysiology of depression, although it makes intuitive sense that symptoms of anhedonia, reduced energy and reduced motivation, which are prominent in many depressed patients, involve this brain reward region (Nestler et al., 2002; Pliakas et al., 2001). Within the amygdala, the consequences of alterations in CREB function appear to be state-dependent. Virus-mediated expression of CREB in the amygdala before training in the learned helplessness paradigm causes depressive-like effect, whereas expression after training results in antidepressant-like effect (Wallace et al., 2004). These finding provide further evidence that the actions of CREB are regionally and temporally specific. Therefore, the increased CREB function within the hippocampus produces antidepressant-like effects that correlate with elevated expression of 
growth factors such as BDNF, whereas the same increases in CREB function within the NAc produce many depressive-like signs. Such observations highlight the fact that CREB functions generally regulate plasticity, a process that is not good or bed; it could be adaptive, maladaptive, or both simultaneously. In the case of depression, elevations in CREB activity in one region and reduction in another could detract from the therapeutic action of treatment regimens that produce global influences on CREB function in the brain (Carlezon et al., 2005).

\subsection{Neurocircuitry of depression}

Regions within the orbital PFC (oPFC) and the MPFC appear to work as a coordinated unit to integrate sensory information, provide emotional salience and modulate visceral motor reactions and value-based decision processes (Wallis, 2011). These regions have connections with several sensory areas (Carmichael and Price, 1995), as well as inputs from the hypothalamus, amygdala, NAc and hippocampus. In rodents, the infralimbic PFC (IL-PFC) is believed to have roles similar to those of the oPFC$\mathrm{mPFC}$ networks, by integrating information and modulating visceral reactions that are related to emotional processes through connections with the amygdala, hypothalamus and various brain stem nuclei (Vertes, 2004). Recent work suggests that the IL-PFC also modulates activation of the ventral tegmental area through effects on the amygdala and ventral subiculum, tying the region to subcortical reward-processing networks (Patton et al., 2013). Anhedonia, especially deficits in non-consummatory reward behaviour, is another core symptom of depression. Abnormal activity levels in the PFC-ACC, as well as in the ventral and dorsal striatum, have been reported in depressed patients with anhedonia (Pizzagalli, 2014).

Depression is also highly heritable, with roughly $40-50 \%$ of the risk for depression being genetic, although the specific genes that underlie this risk have not yet been identified. The remaining $50-60 \%$ of the non-genetic risk also remain poorly defined, with suggestion that early childhood trauma, emotional stress, physical illness, and even viral infections might be involved. Therefore, the highly variable compilation of symptoms that is used to define depression, and the highly variable course of the illness and its response to various treatments, indicate that depression subsumes numerous disease states of distinct etiology, and perhaps distinct pathophysiology. In fact, the lack of bona fide objective diagnostic test for depression, beyond a compilation of symptoms, means that the diagnosis of the syndrome is quite variable, with no clear line distinguishing people who have mild clinical depression from those who are simply having a tough time in the course of normal life (Berton and Nestler, 2006).

\subsection{Animal Models used to screen antidepressant compounds}

Despite the prevalence of depression and its serious impacts, studies on the pathogenesis of depression are still preliminary compared to those on the pathogenesis of other common chronic and potentially fatal multi-factorial conditions (Yang et al., 2010); the major obstacle is the restricted availability of validated animal models.

Ideally, an appropriate animal model of human depression should fulfill the following criteria as much as possible: strong phenomenological similarities and similar pathophysiology (face validity), comparable etiology (construct validity), and common treatment (predictive validity) (Vollmayr et al., 2007; Willner and Mitchell, 
Histaminergic neurotransmission as a gateway for the effects of Oleoylethanolamide

2002). Unfortunately, depression is a heterogeneous disorder and its many core symptoms (e.g., depressed mood, feeling of worthlessness, and recurring thoughts of death or suicide) are hard to be mimicked in laboratory animals.

It should be noted that there is a difference between a model and a test. A model can be defined as an organism (non-human) or a particular state of an organism that reproduces aspects of human pathology, providing a certain degree of predictive validity. A test, on the other hand, provides only an end-point behavioural or physiological measure (read-out) designed to assess the effect of a genetic, pharmacological or environmental manipulation (Urani et al., 2005).

While symptoms of depression are likely to be purely human features, other aspects of the depressive syndrome have been replicated in laboratory animals, and in several instances ameliorated with antidepressant treatment. These include measures of helplessness, anhedonia, behavioural despair and other neuro-vegetative changes such as alterations in sleep and appetite patterns (Krishnan and Nestler, 2011). Many of the test commonly used to detect antidepressant compound are essentially validated retrospectively based on the effects seen with clinically effective antidepressant agents, it is perhaps more appropriate to consider them as a test of antidepressant action rather than test of depression (Cryan and Mombereau, 2004).

I will mention only the most widely used preclinical tests used to screen antidepressant compounds.

\subsubsection{Tail Suspension Test (TST)}

The TST is based on the observation that rodents after initial escape- oriented movements, develop an immobile posture when placed in an escapable stressful situation, that in TST involves haemodynamic stress of being hung in an uncontrollable fashion by their tail (Thierry et al., 1986). If antidepressant treatments are given prior to the test, the subjects will actively persist engaging in escape-directed behaviours for longer periods of time than after vehicle treatment. The test is usually quite short, 6 minutes, and the amount of time they spend immobile is recorded (Steru et al., 1985). Similar to the FST, its validity is questioned by the fact that acute antidepressant treatments reverse the behavioural "depression" (Cryan and Mombereau, 2004). Although both the FST and TST are similar in the construct that they purport to asses, they are probably different in term of the biological substrates that underlie the observed behaviour although they often offer converging data on potential antidepressant (Bai et al., 2001; Porsolt, 2000; Renard et al., 2003).

\subsubsection{Forced Swim Test (FST)}

The Porsolt test (Porsolt et al., 1977), also known as the FST test is the most widely and most frequently used experimental paradigm to detecting antidepressant activity, largely due to its relative reliability across laboratories and its ability to detect activity in a broad spectrum of clinically effective antidepressant (Cryan et al., 2002; Porsolt, 2000). The test is based on the observation that rodents, following initial escape-oriented movements, develop an immobile posture in an inescapable cylinder filled with water. If antidepressant treatments are given prior to the test, the subjects will actively persist engaging in escape-directed behaviours for longer periods of time than after vehicle treatment (Cryan and Mombereau, 2004). 
An interpretation of these tests as a model of depression is that immobility time is a symptom of reduced reactivity to an aversive environment, and the fact that the administration of an antidepressant prolongs the struggling or swimming time should give predictive validity to the model. A main criticism of this interpretation is that a very short-term treatment (acute or short-term 3-4 administration within $24 \mathrm{~h}$ ) is sufficient for shortening immobility time, and this fact conflicts with the delay necessary for an antidepressant compound to develop its therapeutic activity.

There are several differences between the two tests, such as their differential sensitivity to the immobility-reducing effect of various antidepressant with an apparent increased sensitivity of the TST. The mouse FST has not traditionally been viewed as a consistently sensitive model for detecting SSRIs activity (Porsolt and Lenegre, 1992) whereas these antidepressant are generally reported as active in the TST (Cryan et al., 2005).

Despite the major disadvantage of the TST related to its sensitivity to short-term antidepressant, the validity of the TST is based on the behavioural responses, comprising an evolutionary preserved coping strategy in which immobility behaviour represent the psychological concept of "entrapment" described in clinical depression (Dixon et al., 1998; Lucki, 2001). It is reasonably analogous to the human disorder in its manifestation or symptomatology, there are behavioural changes that can be objectively monitored, the behavioural changes observed should be reversed by the same treatment modalities that are effective in humans (Cryan et al., 2005). This fact would imply that TST should be considered a good model of antidepressant action.

\subsubsection{Chronic Mild Stress (CMS)}

One of the most elegant long-term models of depression is the chronic mild stress procedure devised by Willner (Papp et al., 1996; Papp et al., 1991). As the name suggests this paradigm consists of exposing rodents to a series of mild unpredictable stressors during a prolonged period (usually $>2$ weeks). This stress regimen induces many long-term behavioural, neurochemical, neuroimmune and neuroendocrine alterations resembling dysfunctions observed in depressed patients (Willner, 1997). In the CMS model, chronic sequential exposure to a variety of mild stressors has been shown to decrease the drinking of a sweetened solution, a condition that could be reversed by the chronic administration of classical antidepressant drugs as well as dopaminergic agonists (Muscat et al., 1992; Muscat et al., 1990; Papp et al., 1996; Willner et al., 1992). Exposure to chronic mild stress also impairs the acquisition of place preference conditioning, in parallel with sucrose consumption (Papp et al., 1991). These anhedonia-like behaviours have generally been shown to be reversed by chronic, but not acute, treatment with several classes of antidepressants (Moreau et al., 1992; Willner, 1997). Although the paradigm has been described as a model with a high predictive, construct and etiological validity (Cryan and Mombereau, 2004; Willner, 1990). Nevertheless, reservations concerning the reproducibility of the behaviour results obtained have been raised, thus questioning the reliability of the model. 
Histaminergic neurotransmission as a gateway for the effects of Oleoylethanolamide

\subsubsection{Learned Helplessness}

This paradigm was originally developed based on the observations that dogs subjected to repeated inescapable uncontrollable (but not those subjected to controllable) shocks demonstrate escape deficits (Overmier and Seligman, 1967; Seligman and Maier, 1967). The model was later translated to the rat (Seligman and Beagley, 1975) and subsequently to the mouse (Anisman et al., 1979). The rodent studies revealed that the behavioural deficits are sensitive to a broad spectrum of antidepressants usually after short-term treatment (Leshner et al., 1979; Martin et al., 1990a; Martin et al., 1990b; Petty and Sherman, 1979). The major drawbacks of the learned helplessness model are two-fold: first most of the depression-like symptomatology does not persist beyond 2-3 days following cessation of the uncontrollable shock (Weiss and Kilts, 1998). Further, only a certain percentage (estimates vary somewhere between 10 and 80\%) of animals develop helplessness behaviour (Drugan et al., 1989).

\subsubsection{Novelty-suppressed feeding and sucrose preference}

Novelty-suppressed feeding (NSF) test is a hyponeophagia-based behavioural test and provides an anxiety-related measure that is sensitive to the effects of chronic treatment with AD. The NSF also exhibits considerable potential as animal models for studying the neurobiology of the AD response (Dulawa and Hen, 2005). The NSF elicits competing motivations: the drive to eat and the fear of venturing into the centre of a brightly lit arena. Chronic, but not acute treatment with AD could significantly decrease the animal's latency to eat, while it does not affect the food intake of animals in their home cage (Santarelli et al., 2003).

The sucrose preference test is usually used for analysing the effect of drugs on depressive animals. Reduced preference for sweet solution in sucrose preference test represents a loss of interest, fatigue and a loss of energy during depressive episodes, while this reduction can be reversed by treatment with ADs. The reduced sucrose preference has been used as a measure of anhedonia in the animal models of CMS and learned helplessness (Vollmayr et al., 2004; Willner et al., 1987).

\subsubsection{Neuronal histamine: an insight on depression}

Pharmacological or genetic loss of histamine or histamine receptor function in animals produces phenotypes that model human depression (Dai et al., 2007; Ito et al., 1999; Nath et al., 1988; Song et al., 1996). Histamine neurons in the TMN are sensitive to many, if not all, neuroendocrine signals implicated with depression, including biogenic amines, peptides, and steroid hormones, as well as antidepressant medication. Histamine neurons are strongly excited through $5-\mathrm{HT}_{2} \mathrm{C}$, a serotonin receptor that undergoes posttranscriptional editing (Sergeeva et al., 2007) that correlates with suicide (Schmauss, 2003). Noradrenergic $\alpha 2$-receptors increase GABAergic inhibition of TMN neurons (Nelson et al., 2003; Stevens et al., 2004), and interactions with peptidergic influences, e.g., hypocretins (Eriksson et al., 2001; Eriksson et al., 2004), CRH, and steroid hormones, may be implicated in neuroendocrine and coping abnormalities in depression.

PET studies using $\left[{ }^{11} \mathrm{C}\right\rceil$ doxepin, an antidepressant with high affinity to $\mathrm{H}_{1} \mathrm{R}$, revealed reduced $\mathrm{H}_{1} \mathrm{R}$ binding in frontal and prefrontal cortices, and the cingulate gyrus 
correlating with the severity of clinical depression (Kano et al., 2004; Yanai and Tashiro, 2007). Anomalies in histamine metabolism (methylation) may account for endogenous depression in humans (Gagne et al., 1982), and the association of depression and atopy (Timonen et al., 2003) is in line with convergent roles of histamine in immune and stress responses (Steinman, 2004; Theoharides and Konstantinidou, 2007).

Munari and coworkers (2015) demonstrated that an intact histaminergic neurotransmission is essential for the SSRI citalopram and paroxetine to exert their behavioural and neurochemical actions in the TST paradigm. Munari et al., help to clarify also the putative interactions between the serotonergic and histaminergic systems in the antidepressant action of this two compound suggesting that SSRIs increase extracellular levels of endogenous 5-HT in the TMN, which in turn impacts 5$\mathrm{HT}_{2 \mathrm{C}}$ receptors localized on HA neurons, enhances their firing rate, and consequently augments HA release in the cortex. Disruption of this loop in HA-deprived mice is at least in part responsible for the inefficacy of citalopram and paroxetine (Munari et al., 2015).

Single nucleotide polymorphism of the $\mathrm{HA} \mathrm{H}_{1} \mathrm{R}$ gene was found to play a role in bipolar disorder, as it was significantly associated with improvements following olanzapine and fluoxetine treatment (Perlis et al., 2010). In addition, functional mutation in the HDC gene resulting in deficits of the histaminergic neuronal system has been linked to the mechanism and modulation of Tourette's syndrome and tics (Baldan et al., 2014; Ercan-Sencicek et al., 2010). Similar genetic variations in the population may contribute to individual differences in antidepressant response and may prove good predictors of more effective treatments (Munari et al., 2015). Modulation of histaminergic system may thus prove to be useful in the treatments of depression and related mood disorders. 



\section{Chapter 5}

\section{Is neuronal histamine involved in stress-related responses?}

\subsection{Historical Background}

Aristotle, Hippocrates, and the other Ancients were aware of stress and its adverse effects. However, Claude Bernard was the first to formally explain how cells and tissues in multicelled organisms might be protected from stress. Bernard, first pointed out (1859) that the internal medium of the living organism is not merely a vehicle for carrying nourishment to cells. Rather, is the condition of life. Fifty years later, Cannon, working at Harvard, suggested the designation homeostasis for the coordinated physiological processes that maintain most of the steady states in the organism. Cannon coined the term 'fight or flight' to describe an animal's response to threat. The concept of 'fight or flight' proposes that animals react to threats with a general discharge of the sympathetic nervous system, priming the animal for fighting or fleeing. This response was later recognized as the first stage (acute stress response) of a general adaptation syndrome (GAS) postulated by Hans Selye to be a universal stress response among vertebrates and other organisms. Selye, also known as the "father of stress", observed that patients with a variety of illnesses had many of the same "nonspecific" symptoms that were a common response to stressful stimuli experienced by the body. These clinical observations together with experiments on laboratory rats underpinned Selye's concept of GAS, which led Selye to assert that prolonged exposure to stress resulted in "diseases of adaptation". That is, chronic stress, by causing the overproduction of chemicals and hormones, produced gastroduodenal ulcers and high blood pressure. Although the GAS hypothesis was subsequently shown to be incorrect, it did put stress on the map and also highlighted the fact that stress had major effects on the immune system as well as on the adrenal glands. In addition to providing the first clear definition of stress, Hans Selye was also the first to recognize that homeostasis could not by itself ensure stability of body systems under stress. A different tack, focused on cognition, was taken by Lazarus, the eminent and influential Berkeley University psychologist. At a time when psychology tried to understand human behaviour by first understanding simple organisms engaging in simple behaviours learned by associations, rewards, or punishments, Lazarus instead emphasized the importance of studying cognition, which he extended into stress and coping. In parallel with these stress concepts, during the years, neuroendocrine advances revealed the physiological substrate for homeostasis, allostasis, and the stress response mechanisms. The autonomic nervous and the hypothalamic-pituitary-adrenocortical (HPA) systems affect the afferent and efferent limbs of the stress response in vertebrates and are also central to maintaining homeostasis and effecting allostasis (Fink, 2009). 
Histaminergic neurotransmission as a gateway for the effects of Oleoylethanolamide

\subsection{Definition and Classification of Stress}

The term "stress", as it is currently used was coined by Selye in 1936, who defined it as "the non-specific response of the body to any demand for change". Threats to well-being, whether physical or psychological, are components of life experience. Individuals differ markedly, however, in the frequency with which they experience stressful life events and their vulnerability or resilience to stressful challenges (Herman et al., 1995). Stress, although often studied as a psychological construct, may be viewed from a biological perspective (Dantzer, 1991). Accordingly, stress responses are composed of the activation of neurobiological systems that help preserve viability through change or allostasis (McEwen and Seeman, 1999). Although necessary for survival, the effects of frequent physiological stress responses may increase the risk of future physical and mental health problems (Gunnar and Quevedo, 2007).

The Diagnostic and Statistical Manual of Mental Disorders (DSM-V) recognizes two stress disorders: acute stress disorder and posttraumatic stress disorder (PTSD). For the diagnosis of acute stress disorder, the individual, while experiencing the trauma or after the event, must have at least three of several dissociative symptoms, such as a subjective sense of numbing, detachment, or absence of emotional responsiveness; reduction in awareness of surroundings; depersonalization; or dissociative amnesia. Following the trauma, the traumatic event is persistently re-experienced, the individual avoids stimuli that may arouse recollections of the traumatic event, and they have anxiety or increased arousal. PTSD is defined as a condition in which a traumatic event is persistently re-experienced in the form of intrusive recollections, dreams, or dissociative flashback episodes. Cues to the event lead to distress and are avoided, and there are symptoms of increased arousal; the full symptom picture must be present for more than one month, and the disturbance must cause clinically significant distress or impairment in social, occupational, or other areas of functioning (Fink, 2009).

\subsection{Neuroanatomy and Physiology of Stress}

Stress responses in mammals are affected by two distinct, but interrelated systems: the sympathetic-adrenomedullary (SAM) (Frankenhaeuser et al., 1986) system and the hypothalamic-pituitary-adrenocortical (HPA) (Stratakis and Chrousos, 1995) system. The SAM system is a component of the sympathetic division of the autonomic nervous system, releasing epinephrine (adrenaline) from the medulla or centre of the adrenal gland. Increases in circulating epinephrine facilitate rapid mobilization of metabolic resources and orchestration of the fight/flight response (Jansen et al., 1995). The HPA system, in contrast, produces glucocorticoids (cortisol in humans, corticosterone in rodents; hereafter GCs) which are steroid hormones. Unlike epinephrine, which does not cross the blood-brain barrier to a significant degree, the brain is a major target of GCs (Bohus et al., 1982). Also unlike epinephrine, GCs production takes some time (approximately 25 minutes to peak levels), and many of its impacts on the body and brain occur through the changes in gene expression (de Kloet et al., 1991). Consequently, the impacts of GCs are slower to develop and continue for longer periods (De Kloet et al., 1996). The role of the HPA system in stress is complex, and its functions are not fully captured by reference to the fight/flight response (Sapolsky, 2000). Regulation of both the SAM and HPA systems converges at the 
level of the hypothalamus, which integrates autonomic and endocrine functions with behaviour (Palkovits, 1987). Furthermore, inputs to the hypothalamic nuclei that orchestrate HPA and SAM responses to psychosocial stressors involve cortico-limbic pathways (Gray and Bingaman, 1996).

\subsection{Stress: social behaviour, and resilience}

Social stress, a common stressor readily translated across species, is a recurrent factor in the life of all social species (von Holst, 1998). The effects of stress exposure and consequent trajectory depend on the nature of the stressor, the severity, duration (acute vs. chronic), sex/gender, genetics, timing of exposure (early life, adolescence, adulthood or aging) as well as the perception of the stressor by the individual, for example, stressor controllability dramatically affects resilience versus vulnerability as an outcome (Amat et al., 2010; Lucas et al., 2014; Maier and Watkins, 2005). There are three main categories of social stressors. Life events are defined as abrupt, severe life changes that require an individual to adapt quickly. Chronic stressors are defined as persistent events, which require an individual to make adaptations over an extended period of time. When stress becomes chronic, one experiences emotional, behavioural, and physiological changes that can put one under greater risk for developing a mental disorder and physical illness. Understanding the mechanisms underlying stress-induced disturbances will ultimately allow for improved clinical therapies and possible preventative strategies to decrease the incidence of these disorders.

Resilience refers to a person's ability to adapt successfully to acute stress, trauma or more chronic forms of adversity. A resilient individual has thus been tested by adversity (Rutter, 2006) and continues to demonstrate adaptive psychological and physiological stress responses, or "psychobiological allostasis" (Charney, 2004; McEwen, 2003). Resilience has been linked to being able to perceive stressful events in less threatening ways, promoting adaptive coping strategies; such cognitive reappraisal allows individuals to re-evaluate or reframe adverse experiences in a more positive light (Feder et al., 2009; Southwick et al., 2005).

Although many of the above psychological characteristics cannot be measured in animals, some behavioural traits associated with resilience have been identified. In numerous animal models, rodents display a range of responses to stress: at one extreme are active or "fight-flight" responses (for example, attempts to escape and aggression), and at the other extreme are passive responses (for example, freezing and submission). Active-coping animals are often considered to be resilient, based on numerous functional end points, whereas their more passive counterparts are not; however, both types of responses can be seen as adaptive depending on the particular context (Korte et al., 2005).

In rodents, acute stress typically leads to reduced social behaviours and increased aggression, including antisocial behaviours such as bite counts that exceed speciestypical levels (De Almeida et al., 2002; Takahashi et al., 2012). This fits with the concept of acute stress as a 'flight or fight' response and implies that brief acute stressors mobilize resources to cope with the situation (Sandi and Haller, 2015). Chronic stress reduces social motivation and social interactions in a variety of sociability tests (van der Kooij et al., 2014; Wood et al., 2003). However, although chronic stressors generally reduce sociability, social isolation stress actually enhances social interest 
Histaminergic neurotransmission as a gateway for the effects of Oleoylethanolamide

(Van den Berg et al., 1999), probably because long-term deprivation from social contacts increases interest in social partners. Aggressiveness is increased by chronic physical stressors including chronic unpredictable mild stress (Mineur et al., 2003), restraint or immobilization (van der Kooij et al., 2014; Wood et al., 2003; Yohe et al., 2012). By contrast, chronic social stressors that involve fighting that leads to defeat and subordination have been shown to downregulate aggressiveness in various species. Conversely, repeated victories - which are accompanied by reduced physiological stress responses but which can be considered stressful because they involve recurrent exposure to social conflicts - may result in exacerbated and abnormal aggression (Miczek et al., 2013; Nephew and Bridges, 2011).

Stress models that cover a range of neurodevelopmental periods have been applied to investigate the long-term impact of stress on adult social behaviours. Social motivation (sociability) was disrupted in adulthood by prenatal (de Souza et al., 2013), neonatal (Franklin et al., 2011; Yu et al., 2013) and juvenile (Márquez et al., 2013; Naert et al., 2011; Vidal et al., 2011) exposure to stressors. Prenatal stress (de Souza et al., 2013), neonatal stressors -maternal separation (Wei et al., 2013) and early deprivation (Jia et al., 2009) - and peripubertal exposure to physical stressors (Márquez et al., 2013) inhibited social interactions in adulthood. Juvenile social stressors (postweaning social isolation) (Workman et al., 2011) and early subjugation (Wommack et al., 2004) either did not affect this aspect of social behaviour or, in one study (Shimozuru et al., 2008), increased adult social interactions. Early life stressors decrease measures of social motivation, reduce the expression of social behaviours, increase aggressiveness and promote the development of antisocial features, but the specific consequences depend on the timing and type of the early stressor. Although these changes can be problematic for human individuals and societies, from an evolutionary perspective they may be interpreted as mechanisms through which early adversity prepares the organism to endure similar adversities later in life (Gluckman et al., 2007).

\subsection{Stress impact on memory function}

Stress is a potent modulator of cognitive function in general, and more precisely, of learning and memory processes (de Kloet et al., 1999; McEwen and Sapolsky, 1995; Sandi, 2004). Stress effects are frequently regarded as deleterious to cognitive function; but there are many instances in which neural function and cognition are either facilitated by stress (de Kloet et al., 1999; Joëls et al., 2006), or even not affected (Beylin and Shors, 1998; Warren et al., 1991). This great variability can be explained by the "intensity" of the stressor (Cordero et al., 1998) or internal hormonal reactions (Baldi and Bucherelli, 2005; Conrad, 2005; Joëls, 2006). Another important factor is stress "duration," with distinct effects frequently induced by single versus repetitive stress or stress hormones activation, and not only at the cognitive level, but also when evaluating brain structure and function (Pecoraro et al., 2005; Pinnock and Herbert, 2001; Sandi and Loscertales, 1999). Also important to empathize is the memory phase at which stress acts: consolidation is generally facilitated and retrieval generally impaired under stress conditions (Roozendaal, 2002, 2003). Psychological factors, notably stressor controllability and predictability that are well known to be key mediators of the psychophysiological impact of stress (Das et al., 2005; Mineka, 1985) have also to be mentioned addressing the variability of stress on memory processes. Convergent 
evidence indicates that experiencing uncontrollable stress has deleterious effects on further information processing (Maier and Watkins, 2005). To clarify the outcome of stress in memory function is important to take into account the existence of individual differences, with gender appearing as a strong modulator of such interactions (Bowman et al., 2003; Luine, 2002; Shors, 2004). Certainly relevant to understand how stress affects cognition is the relevance of the context in which stress is experienced, that is, whether stress is or not contingent to the particular information processing under study (de Kloet et al., 1999; Joëls et al., 2006; Sandi, 1998).

Chronic stress was also proposed to compromise the hippocampus, a well-known region in the brain important for memory processing (Eichenbaum, 1997; O'Keefe, 1978 ). In the hippocampus, chronically activating the stress response can produce maladaptive changes, which have been postulated to contribute to disease (de Kloet et al., 2005; McEwen and Wingfield, 2003; Smith, 1996). A transition into maladaptive changes includes dendritic remodelling resulting in reduces dendritic arbors in CA3 neurons; dendritic retraction has been observed in other brain regions following ten to 21 days of repeated stress. When chronic stress continues for 4 weeks, CA1 and dentate gyrus neurons express dendritic retraction (Sousa et al., 2000). Prefrontal cortical neurons also express dendritic retraction following 1 to 3 weeks of stress (Brown et al., 2005; Radley et al., 2004). Chronic stress-induced CA3 dendritic remodelling has been proposed to be a maladaptive response because it is associated with susceptibility to damage and cognitive dysfunction (McEwen, 2016).

Although the majority of studies on chronic stress have focused on structural changes within the hippocampus, chronic stress has opposite effects in the basolateral nucleus of the amygdala (BLA), where it increases the dendritic complexity of neurons (Vyas et al., 2002; Vyas et al., 2004), suggesting that chronic stress facilitates memory under emotionally arousing situations.

\subsection{Chronic Stress Paradigm in rodents}

Different animal models have been developed that use chronic stress to induce neuroendocrine and central nervous changes that might reflect also behavioural changes in rodents. The chronic stress paradigms are considered to have a greater etiological relevance and face validity in mimicking depression, anxiety and other diseases.

\subsubsection{Chronic Social Defeat Stress}

Social stressors have proven to be potent across a wide range of species. Social behaviour is complex and varies with the behavioural test chosen, and whether focal individuals are tested with familiar or novel conspecifics, with same- or opposite-sex individuals, or with familiar or unfamiliar strains. Widely used models of social stress in rodents include social subordination, crowding, isolation, and social instability (Figure 4).

Social rejection is used as a potent experimental stressor (Kirschbaum et al., 1993), because individual's position in the social hierarchy has profound implications for health and well-being (Adler et al., 1994; Sapolsky, 2005). The social defeat test procedure involves the daily exposure to a novel, physically superior aggressor for a defined period of time and results in significantly reduced display of social interaction 
Histaminergic neurotransmission as a gateway for the effects of Oleoylethanolamide

and increased anxiety-like behaviour of the defeated animal (van Bokhoven, 2011). Dominance maybe assured by size, prior history of winning, strain of the resident, and/or prior housing differences (Martinez et al., 1998). Social defeat is typically used as a stressor in male rodents, for whom dominance is easier to quantify and aggressive interactions related to home territory are presumed more salient (Beery and Kaufer, 2015). In the short-term, social defeat produces changes in heart rate, hormone secretion, and body temperature, with longer-term impacts on a wide variety of additional outcomes including activity, social behaviour, drug preference, disease susceptibility and others (Martinez et al., 1998; Peters et al., 2012; Sgoifo et al., 1999). Social defeat stress has profound effects on hippocampal morphology and function (Buwalda et al., 2005; McEwen, 2012; McEwen and Magarinos, 2001; Mirescu and Gould, 2006). These effects include reduction in hippocampal volume (Czéh et al., 2001) related to dendritic remodelling and reduced neurogenesis (Gould et al., 1998; Magariños et al., 1996). After prolonged exposure to social defeat stress (and stressful conditions in general), animals often show an altered brain structure and cognition (McEwen, 1999; Sandi and Pinelo-Nava, 2007), that is said to increase the risk of developing neuropsychiatric disorders (de Kloet et al., 2005; Nemeroff et al., 2006)). At the molecular level, BDNF has been reported to play an important role in social defeat stress (Berton and Nestler, 2006). As the key downstream regulatory factor, CREB is a constitutively expressed regulatory nuclear transcription factor involved in not only stress, but also individual development and synaptic plasticity (Guzowski and McGaugh, 1997; Sakamoto et al., 2011).

Social defeat is considered to be one of the most robust animal models of stressinduced mood-related illnesses (Berton and Nestler, 2006). Compared to other animal models, the social defeat paradigm has higher face, predictive, and ethological validity, which results in enduring behavioural and neurobiological changes that mimic several symptoms of the human condition (Iñiguez et al., 2014).

\subsubsection{Crowding and Isolation}

Housing density affects rodent behaviour, and both crowded and isolated social environments have been used as stressors in rodents. Crowding is a naturalistic stressor especially for social or gregarious species that relates to high population density and resource competition in the field. In house mice, several studies have shown that crowding can impair reproductive function and may be part of population size regulation (Christian, 1971; CHRISTIAN and LEMUNYAN, 1958). Increased group size is associated with greater dispersal consistent with a "social competition" hypothesis (Quirici et al., 2011). Social crowding has been shown to affect many different physiological outcomes in rodents. These include changes in organ weights, hormone secretion, HPA reactivity, pain sensitivity, telomere length, and cardiac outcomes (Gadek-Michalska, 2003; Gamallo et al., 1986; Grippo et al., 2011; Kotrschal et al., 2007; Puzserova et al., 2013; Tramullas et al., 2012). At the opposite extreme, solitary housing can be a potent stressor for social species. Solitary housing produces an "isolation syndrome" particularly in females, consisting of hyperadrenocorticism, reduced body weight, altered blood composition, and enhanced pain responsiveness among other outcomes (Hatch et al., 1965; Valzelli, 1973). These changes coincide with alterations in behaviour including aggression, mating behaviour, learning, and pain sensitivity (Valzelli, 1973). 


\subsubsection{Social Instability}

Some studies employ both crowding and isolation in alternation as a model for chronic social instability (Haller et al., 1999; Herzog et al., 2009). In the social instability stress paradigm, uncontrollability is modelled by alternating isolation and crowding phases and by the rotation of animals among social groups during the crowding phase (Herzog et al., 2009). Social instability has particularly been used as a social stressor for female rats, for whom crowding and social defeat are not always effective stressors (Palanza et al., 2001). In the crowding phase, different social groups consisting of different numbers of males and females are formed. Females exposed to this variable social environment show increased adrenal weight, increased corticosterone secretion, decreased thymus weight, and reduced weight gain relative to females housed in stable male e female pairs (Haller et al., 1999). Social instability also induced dysregulation of the hypothalamic-pituitary-gonadal (HPG) axis and reduced sucrose preference and food intake (Herzog et al., 2009).

\subsubsection{Chronic Restrain Stress}

Chronic restrain is induced by placing rodents into a well ventilated transparent tube for a few hours per day for 2-3 weeks. The animals display depressive-like behaviour (Haenisch et al., 2009) and also impairment of hippocampal neurogenesis, down-regulation of neurotrophins, such as BDNF, and alteration in synaptic plasticity markers (Pollak et al., 2010; Veena et al., 2009). However, daily exposure to the same kind of stressor at the same time is experienced as predictable mild stress, which may actually improve mood and enhance hippocampal neurogenesis in mice (Parihar et al., 2011) so that the relevance of this paradigm is questionable if not associated with other stressors.

\subsection{Neuronal histamine: an insight on stress}

Histamine release is a sensitive indicator of stress (Taylor and Snyder, 1971; Verdière et al., 1977), and chronic restraint and/or metabolic stress are among the most potent activators of histamine neurons in the TMN (Miklós and Kovács, 2003). Distinct subgroups (E4-E5) of hypothalamic histamine neurons respond to immobility, foot shock, hypoglycemia, and dehydration, suggesting a functional heterogeneity of histaminergic TMN neurons (Miklós and Kovács, 2003). TMN neurons are influenced by a number of neuroendocrine signals (Gotoh et al., 2005) and may integrate exteroceptive and interoceptive state cues in the control of stress-induced arousal. Histamine mediates the stress-induced neuroendocrine hormone surges of ACTH, $\beta$ endorphin, and AVP from the pituitary (Kjaer et al., 1992) and controls stress-related activity of aminergic systems, including serotonin-, norepinephrine-, dopamine-, and acetylcholine-containing neurons. As an integral part of the neural networks generating autonomic patterns histamine neurons interfere with AVP- and CRH-positive sympathetic command neurons (Krout et al., 2003) in the PVN and LHA (Whitcup et al., 2004) to influence sympatho-adrenal outflow, cardiovascular functions, and complex stress-related behaviours such as 'flight or fight' or grooming. Histamine injections in the PVN activate the HPA axis through CRH release. Moreover, both hista- 
Histaminergic neurotransmission as a gateway for the effects of Oleoylethanolamide mine and $\mathrm{CRH}$ are released from mast cells in the leptomininges and along brain capillaries during systemic stress emphasizing the intricate interaction between histamine and CRH, and the nervous and immune system (Esposito et al., 2002). 


\section{Chapter 6 \\ Histamine and the gut-brain axis}

The bidirectional signalling between the gastrointestinal tract and the brain is vital for maintaining homeostasis and is regulated at the neural (both central and enteric nervous systems), hormonal and immunological levels. Perturbation of these systems results in alterations in the stress-response and overall behaviour (Rhee et al., 2009). The high co-morbidity between stress-related psychiatric symptoms such as anxiety with gastrointestinal disorders including irritable bowel disorder (IBS) and inflammatory bowel disorder (IBD) (Cámara et al., 2009; Mawdsley and Rampton, 2006) are further evidence of the importance of this axis. However, increasing evidence also suggests that the enteric microbiome greatly impacts on gut-brain communication (Cryan and O'Mahony, 2011). The necessary communication processes are based on neurotransmitters, neuropeptides, cytokines, hormones, growth factors (among others), which mediate the relationship between the immune system and the CNS (Downing and Miyan, 2000). Also, luminal probiotic bacteria may alter behaviour and brain biochemistry in a variety of ways even in the absence of changes in the inflammatory status of the host (Bercik, 2011). Bacterial products could enter the circulation to pass the blood-brain barrier if they are sufficiently small and lipophilic, or they might enter the brain at the circumventricular organs where the barrier is diminished. Since prior vagotomy abolishes behavioural and brain biochemical changes induced by certain probiotic bacteria (Bercik et al., 2011; Bravo et al., 2011), afferent vagal signalling is a necessary condition for the central effects of these neuroactive microorganisms.

Under stress conditions, the brain may influence the composition of the gut microbiota (Bailey and Coe, 1999) via the hypothalamus-pituitary-adrenal (HPA) axis, which regulates cortisol secretion, affecting immune cells activity both locally in the gut and systemically. Stress can affect such equilibrium (Glaser and Kiecolt-Glaser, 2005), leading to allergic reactions, inflammatory responses and predisposition to infection. In turn, gut microbiota, and probiotic agents can alter the levels of circulating cytokines, which in turn can have a marked effect on several brain functions (Duerkop et al., 2009; Forsythe and Bienenstock, 2010).

Recent advances in understanding the molecular and systems biology of the gutbrain axis have suggested novel candidates such as gut lipid sensors, and have revealed novel roles for lipid sensing in the control of nutrient availability (Schwartz, 2011) and in cognitive functions. One of these sensors is the endocannabinoid oleoylethanolamide. 
Histaminergic neurotransmission as a gateway for the effects of Oleoylethanolamide

\subsection{Oleoylethanolamide}

The ability to monitor the nutrient composition of food allows animals to generate neural and endocrine feedback signals that adapt behaviour and metabolism to environmental fluctuations in food availability. Being able to sense dietary fat is especially important, from an adaptive perspective, because of the high energy density of this nutrient and the essential role of lipids as building blocks of cell membranes and precursors for hormones and other bioactive molecules. The need to secure appropriate amounts of fat and optimize its storage and utilization provides a plausible evolutionary basis for the existence of multiple lipid-sensing mechanisms throughout the mammalian body (Piomelli, 2013). New evidence suggests an important role for a different class of signalling molecules - the amides of fatty acids (FAs) with ethanolamine (fatty acid ethanolamides, FAEs). The FAEs were first isolated from plant and animal tissues more than 50 years ago (BACHUR et al., 1965; Kuehl, 1957) and were found to possess strong anti-inflammatory activity (Lambert et al., 2002). The discovery of anandamide (AEA), a polyunsaturated FAE led to uncovering the function served by FAE family molecules as regulators of food intake (Rodríguez de Fonseca et al., 2001). This family includes the monounsaturated analogue oleoylethanolamide (OEA) (Piomelli, 2013), which, although sharing similar biosynthetic pathways (Okamoto et al., 2007) with AEA, exerts opposite effects on feeding regulation and lipid metabolism (Romano et al., 2014).

A calcium-dependent $N$-acyltransferase activity (NAT) catalyses the transfer of a fatty acyl group from the $s n-1$ position of phosphatidylcholine to the amine group of phosphatidylethanolamine, producing a family of $N$-acylphosphatidylethanolamine (NAPE) species. NAPEs containing oleic acid at the amine position generate OEA upon hydrolysis of their distal phosphodiester bond. This reaction is catalysed by a NAPE-selective phospholipase D (NAPE-PLD), which produces phosphatidic acid (PA) as a by-product (Figure 6). OEA is hydrolysed into oleic acid and ethanolamine by either of two structurally unrelated enzymes: FA amide hydrolase (FAAH) or $N$ acylethanolamine acid amidase (NAAA) (Piomelli, 2013).

In particular, OEA is synthesized in the upper part of the small intestine, upon the absorption of lipids from the diet (Fu et al., 2007; Li et al., 2015). Endogenous OEA itself is nutritionally regulated specifically by intestinal lipid administration. Food deprivation reduces OEA biosynthesis in the small intestine, while intestinal lipid infusions, but not equicaloric carbohydrate or protein infusions, increase proximal small intestinal OEA levels (Schwartz et al., 2008). Interestingly, the biosynthetic pathway responsible for lipid stimulated endogenous OEA production also generates intra-intestinal oleic acid, bringing full circle an extrinsic food-derived lipid signal to a de novo intra-intestinal oleate signal (Schwartz et al., 2008). Feeding suppressive effects of small intestinal lipid infusions are mediated in part by a local regulatory network within upper small intestine: nutritional lipid is translocated into the luminal tissue and stimulates gut OEA and oleic acid biosynthesis (Schwartz, 2011).

Peripheral OEA administration reduces food intake by increasing the latency to feed and by prolonging the interval between two successive meals (Fu et al., 2005; Gaetani et al., 2003; Rodríguez de Fonseca et al., 2001). OEA hypophagic actions cannot be attributed to stress or malaise because OEA does not produce behaviours that are indicative of fear or anxiety, does not change plasma corticosterone levels, and does not induce conditioned taste aversion in rats (Fu et al., 2005; Proulx et al., 
2005) . The hypophagic actions of OEA depend on the feeding state of the animal. In free-feeding rats, OEA decreases meal frequency without altering meal size; by contrast, the compound simultaneously reduces these two parameters in food-deprived animals (Gaetani et al., 2003).

The effects of OEA on food intake appear to be mediated in part by Peroxisome Proliferator Activated Receptor (PPAR)-alpha, a nuclear receptor that is also implicated in the mechanism that allows liver cells to gauge the levels of circulating FAs (Pawar and Jump, 2003) . OEA fails to suppress feeding in PPAR-alpha deficient mice, while PPAR-alpha agonists mimic the feeding inhibitory effects of exogenous OEA administration (Rodríguez de Fonseca et al., 2001). Gut vagal afferents also contribute to the ability of OEA to reduce feeding, as vagal capsaicin application blocks OEA induced satiety (Rodríguez de Fonseca et al., 2001). Consistent with this finding, OEA also rapidly depolarizes capsaicin-sensitive cell bodies of vagal afferent neurons in the nodose ganglion. This activation is likely mediated by the transient receptor potential vanilloid type 1 (TRPV1) receptor, as it is blocked by TRPV1 antagonists and is absent in TRPV1 null mice (Wang et al., 2005). OEA also acts as an agonist for GPR119 (Suardíaz et al., 2007), a G protein-coupled receptor that recognizes a broad panel of lipid molecules in addition to OEA (Hansen et al., 2012). GPR119 is expressed in intestinal endocrine L-cells, which secrete glucagon-like peptide-1 (GLP-1), and intraluminal infusions of OEA were found to increase circulating GLP-1 levels in rats (Lauffer et al., 2009). Nevertheless, the observation that genetic deletion of TRPV1 or GPR119 in mice does not alter the anorexic effects of OEA strongly argues against a direct involvement of these receptors in OEA-induced satiety (Lan et al., 2009; Lo Verme et al., 2005).

Intraperitoneal injections of OEA stimulate transcription of c-Fos in the Nucleus of the Solitary Tract (NST) and in peptide-secreting neurons of the paraventricular (PVN) and supraoptic (SON) nuclei of the hypothalamus (Gaetani et al., 2010; Rodríguez de Fonseca et al., 2001) and increase the expression of the neuropeptide oxytocin, but not vasopressin. Moreover, pharmacological blockade of central oxytocin receptors abrogates the hypophagic effects of OEA, implying that release of oxytocin in the hypothalamus and/or other regions of the brain may be a key effector of OEA-induced satiety (Gaetani et al., 2010).

OEA recruits other neurotransmitter systems in the brain to reduce food intake. Recently in our laboratory it was shown that histamine deficiency significantly attenuates OEA-induced hypophagia (Provensi et al., 2014). As previously reported in this thesis, brain histamine affects feeding behaviour, it is fundamental for appetitive and aversive responses during motivated behaviour, and blockade of histamine $\mathrm{H}_{1} \mathrm{R}$ in the hypothalamus is believed to be responsible for the weight gain and metabolic dysregulation associated with the clinical use of atypical antipsychotics (Kim et al., 2007). Provensi and co-workers (2014) demonstrated that lack of central histamine dampens OEA-induced increase of c-Fos expression in oxytocin PVN neurons. Therefore, OEA requires the integrity of the brain histamine system to fully exert its hypophagic effect and the finding establish new functional connections between peripherally acting hypophagic signals and brain histamine neurotransmission (Provensi et al., 2014).

OEA exert also other functions mediated at least in part by the intestine-brain connections. By using the inhibitory avoidance and the Morris water maze test, Campolongo et al. (2009) found that i.p. administration of OEA after behavioural training 
Histaminergic neurotransmission as a gateway for the effects of Oleoylethanolamide

strongly improved the retention of these tasks. This effect was blocked by infusions of the local anaesthetic lidocaine into the NST or the $\beta$-adrenergic antagonist propranolol into the BLA, indicating that the signal launched by peripheral OEA gains access to the CNS and strengthens memory consolidation by stimulating noradrenergic activity in the BLA (Campolongo et al., 2009). This suggest that OEA signalling initiates an integrated response in which satiety induced by a fat-rich meal coincides temporally with enhanced encoding of information about the spatial and emotional context in which the meal was consumed highlighting the importance of metabolic peripherally acting signals in the regulation of higher brain function (Berthoud and Morrison, 2008).

Other behavioural effects of OEA include antidyskinetic effect as assessed by using a hemiparkinsonian model of PD in mice with 6-OHDA striatal lesion. OEA treatment reduced axial, forelimb and orolingual dyskinetic symptoms, as well as contralateral rotations induced by 6-OHDA (González-Aparicio and Moratalla, 2014). Also, OEA reduced spontaneous locomotor activity and attenuated psychomotor activation induced by cocaine, an effect that does not seem to be mediated by PPAR $\alpha$ receptor (Bilbao et al., 2013). OEA's central effects were also tested in depressive-like behaviour by using two animal models: the chronic unpredictable mild stress (CUMS) and tail suspension/forced swim tests. OEA treatment normalized sucrose preferences, rearing frequencies, prefrontal cortex and hippocampal atrophy and reversed the abnormalities of BDNF and MDA levels and SOD activities in the hippocampus and prefrontal cortex, as well as changes in serum levels of ACTH, CORT, and T-AOC in CUMS, demonstrating antioxidant properties and normalization of the hyperactivity in the hypothalamic-pituitary-adrenal axis (HPA) (Jin et al., 2015). In the TST/FST, OEA treatment decreased the immobility time demonstrating an antidepressant-like effect and also and increased cerebral levels of NE and 5-HT regulating central monoamine neurotransmitters (Yu et al., 2015a). 


\section{Aim of the study}

The main scope of my study is to understand the role of the histaminergic system in the homeostatic and behavioural effects of oleoylethanolamide (OEA), a fat sensing hormone normally secreted by enterocytes in the jejunum. Our working hypothesis is that brain histamine serves as a relay station that elaborates peripheral and central signals to allocate the relevance and adequate arousal to perform the appropriate behaviour.

For the purpose of clarity, the manuscript is divided in three parts concerning different aspects of the interaction of the histaminergic system and oleoylethanolamide.

In the first part of the work we brain histamine is necessary for OEA to exert its effects on emotional memory. The results appear in the publication Provensi et al., 2017 (Provensi et al., 2017) that I co-authored.

In the second part, I investigated whether histamine has a role in OEA's antidepressant-like effects. In this regard, our research group recently demonstrated that selective serotonin reuptake inhibitors require the integrity of the brain histamine system to exert their preclinical responses (Munari et al., 2015), Consequently we hypothesised a possible relationship between antidepressant-like effect of OEA (Jin et al., 2015) and brain histamine. To this end, we evaluated the repeated and chronic i.p. administration of OEA in mice unable to synthesize HA due to disruption of the histidine decarboxylase gene (HDC-KO) or to injection of alpha-fluoromethylhistidine compared to wild type (WT), and saline i.c.v. injected controls in the tail suspension test. The phosphorylation level of cyclic AMP-response element binding protein (pCREB), a major player in the molecular mechanisms of antidepressant treatment was also evaluated by Western Blot analysis in prefrontal cortex and hippocampus, two regions critically involved in depression and antidepressant effects of drugs.

In the third part of the thesis we investigated the effect of behavioural effects of OEA in chronic social defeat stress (CSDS)-induced cognitive impairments. In 1971 it was reported that brain histamine turnover was altered by stress conditions (Taylor and Snyder, 1971). Stress affects a constellation of physiological systems in the body and evokes a rapid shift in many neurobehavioral processes. In our hypothesis histamine may detect stress-induced signals from the periphery. As the influence of OEA on stress reactivity is not currently addressed, we evaluated the effect of OEA in HDC$\mathrm{KO}$ and wild type mice subjected to the CSDS. In CSDS paradigm experimental mice are exposed to social and physical conflict by aggressive member of the same species resulting in both physical and emotional stress for 21 days, while control non-stressed mice will be handled daily. The behavioural repertoire of the animals has been assessed at the end of the CDSC, using a battery of tests that are comprehensive of several domains affected by stress: social behaviour, mood, anxiety and cognition. 



\section{Results \\ Part I \\ Histaminergic Neurotransmission as a Gateway for the Cognitive Effect of Oleoylethanolamide in Contextual Fear Conditioning}

\subsection{Materials and Methods}

\subsubsection{Animals and Drugs}

Male Wistar rats (3 months old, 300-330 g) purchased from Envigo (Bresso, Italy) were housed in the animal facility of Ce.S.A.L (Università di Firenze) in a temperature-controlled room $\left(22 \pm 1^{\circ} \mathrm{C}\right)$ with a 12-h-light/dark cycle (light on 7:00 AM to 7:00 PM), at a constant temperature and humidity with standard diet (4RF21; Mucedola s.r.l., Milan, Italy) and freely available water. All procedures were conducted in accordance with the Council Directive of the European Community (2010/63/EU) of the Decreto Legislativo Italiano 26 (13/03/2014) and National Institutes of Health guidelines on animal care and were approved by veterinarian supervision.

Alpha-FMHis was synthesized at Johnson \& Johnson Laboratories (kind gift of Dr. Nicholas Carruthers), pyrilamine was purchased from Sigma-Aldrich (UK), and zolantadine and OEA from Tocris Bioscience (UK). OEA was dissolved in saline/polyethylene glycol/Tween $80(90 / 5 / 5, \mathrm{v} / \mathrm{v})$, whereas zolantidine and pyrilamine were dissolved in saline. All other reagents and solvents were of HPLC grade or the highest grade available (Sigma).

\subsubsection{Surgery}

One week after arrival, rats were anaesthetized $(75 \mathrm{mg} / \mathrm{kg}$ ketamine plus $10 \mathrm{mg} / \mathrm{kg}$ xylazine) and placed on a stereotaxic frame (Stellar; Stoeling, Co., Wood Dale, IL). A stainless-steel cannula (22 gauge) was implanted in the lateral ventricle and fixed to the skull by using dental cement according to the following coordinates (Paxinos et al., 1998) in mm: $\mathrm{AP}=-0.9 ; \mathrm{L}=-1.5 ; \mathrm{DV}=-2.6$ and used for $\alpha$-FMHis/saline administration. Rats were also implanted bilaterally with 22 -gauge guide cannulae $1 \mathrm{~mm}$ above the BLA according to the following coordinates from bregma (Paxinos et al., 1998) in $\mathrm{mm}: \mathrm{AP}=-2.8 ; \mathrm{L}= \pm 4.9 ; \mathrm{DV}=+7.6$. Animals were allowed 7 days to recover from surgery before behavioural procedures and were handled once daily before the experimental day. 
Histaminergic neurotransmission as a gateway for the effects of Oleoylethanolamide

\subsubsection{Infusion Procedure and Experimental Groups}

At the time of drug microinfusions, the animals were gently restrained by hand, and the injection needle ( 30 gauge) was fitted tightly into the guides, extending $1 \mathrm{~mm}$ from the tip of the guide cannulae. The injection needle was connected to a $10-\mu \mathrm{L}$ Hamilton microsyringe, and the infusions were performed at a rate of $0.5 \mu \mathrm{L} / 30 \mathrm{~s}$. The infusion cannula was left in place for an additional 60 seconds to minimize backflow. The entire bilateral infusion procedure took approximately 90 seconds. Alpha-FMHis $(5 \mathrm{mM}, 1 \mu \mathrm{L})$ was infused i.c.v. 24 hours before contextual fear training, and controls received equal volumes of sterile saline. Zolantidine $(0.1 \mu \mathrm{M}, 0.5 \mu \mathrm{L}$ side) or pyrilamine $(0.9 \mu \mathrm{M}, 0.5 \mu \mathrm{L}$ side $)$ were infused intra-BLA bilaterally immediately after training. OEA $(10 \mathrm{mg} / \mathrm{kg})$ was injected i.p. 10 minutes after fear conditioning, while controls received equivalent volumes of vehicle.

\subsubsection{Contextual Fear Conditioning}

Contextual fear conditioning was induced in a Skinner box module $(29 \times 31 \times 26$ $\mathrm{cm}$, Modular Operant Cage; Coulbourn Instruments Inc.), equipped with a grid floor connected to a shock-delivery apparatus (Modular Operant Cage/Grid Floor Shocker E13-08; Coulbourn Instruments) and placed in an acoustically insulated room at $20 \pm 1{ }^{\circ} \mathrm{C}$. The number of the electric shocks and the inter-shock interval duration was predetermined by a stimulus programming device (Scatola di comando Arco 2340, Italy). Illumination inside the room was 60 lux. The rat was left undisturbed for 3 minutes and subsequently six, 1 -seconds $0.8-\mathrm{mA}$ electric footshocks were administered at 30 -second intervals. The footshock intensity was chosen according to previous published data from our laboratory (Benetti et al., 2013). This is a strong enough footshock to guarantee retention at 72 hours postacquisition without inducing generalization (Baldi et al., 2004). The rat was removed 2 minutes after the end of the stimulation, therefore spending a total time of 8 minutes inside the conditioning apparatus.

\subsubsection{Freezing Measurement}

Seventy-two hours after conditioning, rats were again placed inside the conditioning apparatus in the soundproof room and left undisturbed for 6 minutes. The rats' behaviour was recorded by means of a closed-circuit television system by an experimenter unaware of the animal's treatment. Freezing was defined as the complete absence of somatic motility, with the exception of respiratory movements. Measurements were performed with a stopwatch by personnel unaware of the experimental group each animal belonged to. Total cumulated freezing time (i.e., total seconds spent freezing during each 6-minute period) was calculated and results expressed in seconds of freezing time. All behavioral tests were performed between 10:00 AM and 12:00 PM to avoid interference with the circadian rhythm (Kamin, 1957).

\subsubsection{Histology}

The placement of infusion cannulae was verified postmortem. Rats were overdosed with chloral hydrate and the brains removed and stored in $10 \%$ formalin for 10 days. Forty- $\mu \mathrm{m}$ sections were sliced on a cryostat, mounted on gelatine-coated slides, 
and then stained with cresyl violet for light microscopic observation. Data from rats in which the cannulae were not correctly positioned were discarded $(<5 \%)$.

\subsubsection{Statistical Analysis}

All values are expressed as means \pm SEM, and the number of rats used in each experiment is also indicated. The presence of significant treatment effects was determined by a Student's $t$ test or a 1-way ANOVA followed by Newmann Keuls' MCT test, as appropriate. For all statistical tests, $P<.05$ was considered significant.

\subsection{Results}

\subsubsection{Oleoylethanolamide administration increases freezing time of rats submit- ted to contextual fear conditioning.}

In a first set of experiments we evaluated the effect of OEA $(10 \mathrm{mg} / \mathrm{kg}$ i.p. a dose that does not change motility in the open filed, nor anxiety-like responses, (Campolongo et al., 2009) administered within 10 min of contextual fear conditioning in satiated rats. Controls received an equivalent volume of vehicle. Retention test was carried out 72 hrs after training. As shown in figure 7, an unpaired Student' t test showed that rats given OEA displayed a significant increase of time spent freezing compared with vehicle treated animals $(\mathrm{p}<0.01)$.

\subsubsection{Histaminergic neurotransmission is required for OEA-freezing enhance- ments}

To evaluate the role of the central histaminergic system in the cognitive effect of OEA, we infused the HDC inhibitor $\alpha$-FMHis i.c.v. 24 hours prior to fear conditioning. Our research group previously showed that administration of $\alpha$-FMHis quickly suppressed baseline and histamine $\mathrm{H}_{3}$ receptor antagonist-evoked release of histamine from the TMN of freely moving rats (Benetti et al., 2015), as 180 minutes after injection, histamine release values decreased below the sensitivity of the method. OEA (10 $\mathrm{mg} / \mathrm{kg}$ i.p.) was injected within 10 minutes after fear conditioning. Controls received saline i.c.v. and vehicle i.p. One-way ANOVA revealed a statistical difference across experimental groups $\left(\mathrm{F}_{3,45}=6.756 ; P<.001\right.$; Figure 8$)$. Post-hoc analysis with Newman-Keuls MCT showed that OEA increased the freezing time at retention test with respect to vehicle-treated rats receiving i.c.v. infusion of saline $(P<.05)$. However, the OEA-elicited potentiation of freezing was abolished in brain histamine-depleted animals $(P<.01)$. Hence, the treatment with $\alpha$-FMHis 24 hours prior to the test prevented the effect of OEA, indicating that the integrity of the central histaminergic system is necessary for the effects of OEA on memory consolidation. The freezing time of rats given vehicle i.p. and of those receiving $\alpha$-FMHis or saline i.c.v. did not differ significantly at retention, thus indicating that all animals formed a memory trace of the training experience. 
Histaminergic neurotransmission as a gateway for the effects of Oleoylethanolamide

\subsubsection{Antagonism of histamine $\mathrm{H}_{1}$ and $\mathrm{H}_{2}$ receptors prevents OEA-induced freezing enhancement}

Figure 9 shows the effect of blocking $\mathrm{H}_{1}$ or $\mathrm{H}_{2}$ receptors in the BLA with local, bilateral infusions $(0.5 \mu \mathrm{L})$ of the selective $\mathrm{H}_{1}$ antagonists pyrilamine $(0.9 \mu \mathrm{M})$ or the $\mathrm{H}_{2}$ antagonist zolantidine $(0.1 \mu \mathrm{M})$, immediately prior to the administration of OEA. Controls received intra-BLA, comparable volumes of saline and i.p injections of vehicle. One-way ANOVA showed significant differences among experimental groups $\left(\mathrm{F}_{5,64}=8.436 ; P<.0001\right)$. Rats that received OEA i.p. and saline intra-BLA froze for a significantly longer time at retention compared with saline-/vehicle-treated rats $(P<.05)$ in a comparable manner to the control groups in Figure 8. However, when either $\mathrm{H}_{1}$ or $\mathrm{H}_{2}$ receptors were blocked, OEA administration did not increase the freezing time $(P<.001)$. Freezing time of rats that received vehicle i.p. and pyrilamine or zolantidine in the BLA was not significantly different from the freezing time of saline-/vehicle-treated rats.

\subsection{Summary of Results (Part I)}

Emotional arousal enhances consolidation of memory traces, a homeostatic response of our organism that is modulated by stress hormones (McGaugh and Roozendaal, 2002). There is extensive evidence derived from observations in both experimental animals and humans that the amygdala is indispensable to enable the acquisition and retention of lasting memories of emotional experiences. The compelling evidence led McGaugh and his collaborators (McGaugh, 2004) to propose that the activation of the BLA is fundamental in the establishment of an arousal state triggered by fear as unconsciously occurs in humans, (LeDoux, 2014) and that arousal is a major component in the establishment of post-training memory consolidation.

By activating peripheral PPAR- $\alpha$ OEA engages the vagus nerve and activates the NTS that in turn provides the amygdala, together with the locus coeruleus (LC), with a dense supply of norepinephrine, and modulates long term memories (Campolongo et al., 2009).

The present results show that OEA increases memory expression when tested in the contextual fear conditioning paradigm, by increase the freezing time of treated animals compared to vehicle injected rats. Depletion of histamine in the brain with infusion of $\alpha$-FMHis that blocks HDC, or intra-BLA infusions of antagonists of the $\mathrm{H}_{1}$ or $\mathrm{H}_{2}$ receptor prevent the freezing-enhancing effects of OEA. Therefore, the histaminergic system in the amygdala exerts a permissive role on the memory-enhancing effects of OEA. When histaminergic neurotransmission is compromised by $\alpha$-FMHisinduced depletion OEA effects on memory expression is prevented. It is relatively surprising that both $\mathrm{H}_{1}$ and $\mathrm{H}_{2}$ receptor antagonists produce the same results. The arrangement of these receptors on BLA neurons is not known, and explanations may only be speculative. Presumably, when strong aversive stimuli are used, all histaminergic inputs to the BLA need to be blocked to prevent freezing-enhancing compounds such as OEA to exert their effect.

The concept of several neurotransmitter systems contributing to emotional memory consolidation within the same brain region is indisputable (Izquierdo et al., 1992; Izquierdo et al., 2016; McGaugh, 2004). We think that the emotional arousal that is generally considered indispensable for good memory consolidation of fear tasks 
is provided by both the noradrenergic and histaminergic transmission in the BLA. In our hypothesis histamine released in the amygdala gates the effects of noradrenaline on BLA neurons, hence blocking either neurotransmitter within the BLA would lead to similar behavioural outcomes. In this case, $\mathrm{H}_{1}$ and $\mathrm{H}_{2}$ receptors work synergistically presumably activating common intracellular pathways and their blockade leads to similar behavioural outcomes.

Taking into consideration the results presented in this thesis and previous evidence (Campolongo et al., 2009), OEA appears to increase the expression of BLAdependent emotional memory regardless of the type of behavioural response and adverse situation. From a translation point of view, few human studies published so far showed that blood concentrations of $\mathrm{N}$-acylethanolamides like OEA and other endocannabinoids increase in response to acute stress in healthy human volunteers (Dlugos et al., 2012), and subjects affected by Post-traumatic stress disorder (PTSD) have significantly higher plasma concentrations of OEA (Hauer et al., 2013; Schaefer et al., 2014). These results are based on small and dis-homogeneous cohort of patients, nonetheless they may have diagnostic relevance. In this regard, the results presented in this thesis, suggest that activation of the histaminergic system in the BLA has a "permissive" role on the memory enhancing effects of OEA. In particular, targeting the $\mathrm{H} 1$ or $\mathrm{H} 2$ receptor with classical clinically approved antihistamine compounds may modify the expression of emotional memory and reduce dysfunctional aversive memories as found in phobias and PTSD. 



\section{Part II \\ Oleoylethanolamide induces antidepressant-related responses by targeting PPAR- $\alpha$ and recruiting the histaminergic neurotransmission}

\subsection{Materials and Methods}

\subsubsection{Animals and Drugs}

Male CD1 mice (25-35 g; Harlan, Italy), histidine decarboxylase knockout (HDCKO) mice and wild type (WT) littermates (background sv129), grown in the Centro Sabulazione Animali di Laboratorio (CeSAL), Università di Firenze were used for behavioural and biochemical experiments. Genetically modified animals were routinely genotyped using the PCR protocol previously described (Provensi et al., 2014). Peroxisome proliferator activated receptor-alpha knockout (PPAR- $\alpha-\mathrm{KO})$ mice and wild type (WT) littermates (background C57Bl/6), were used for behavioural and biochemical experiments. Wild-type and PPAR- $\alpha$ KO (B6.129S4-SvJaePparatm 1Gonz) mice previously backcrossed to C57BL6 mice for 10 generations were bred in Centro Stabulazione Animali di Laboratorio, Università Federico II di Napoli, and the colony was established and maintained by heterozygous crossing. Mice were genotyped as described on the supplier webpage (http://jaxmice.jax.org). Animals were housed in humidity and temperature-controlled room $\left(22-24{ }^{\circ} \mathrm{C}\right)$, allowed free access to food (4RF21; Mucedola s.r.l., Milan, Italy) and water, and kept on a 12-h light/dark cycle (lights start at 7:00 AM). All the experiments were conducted between 9:00 AM and 4:00 PM. OEA and vehicle were dissolved as previously reported (Result part I). Imipramine (IMI) was dissolved in saline. Acute neuronal histamine deprivation in CD1 mouse was induced by injection intra-cerebro-ventricular (i.c.v) of $5 \mu \mathrm{L}$ of $\alpha$-fluoro-methyl-histamine solution $(1 \mu \mathrm{g} / \mu \mathrm{L})$. The control group received an injection of $5 \mu \mathrm{L}$ of saline ic.v..

\subsubsection{Behavioral Experiments}

\subsubsection{Tail Suspension Test (TST)}

Mice were fixed with a $1 \mathrm{~cm}$ piece of adhesive tape from the tip of the tail, suspended $50 \mathrm{~cm}$ from the ground, at least $30 \mathrm{~cm}$ away from the objects that may surround them and the behavior was filmed by a camera positioned in front of the apparatus for $6 \mathrm{~min}$. The immobility time was manually timed by a researcher unaware of the treatment. OEA was administered following two different regimens: $10 \mathrm{mg} / \mathrm{kg}$ 
Histaminergic neurotransmission as a gateway for the effects of Oleoylethanolamide

OEA repeated administration, 3 i.p. injections in 24 hours and $10 \mathrm{mg} / \mathrm{kg}$ i.p. OEA subchronic with 1 daily i.p. injection for 8 days before challenging the mice in TST $1 \mathrm{~h}$ after the last treatment. The test was carried out in a soundproof room with low light and was recorded using a video camera (Digital Cam 34x, Samsung). The time that mice remained immobile (immobility time) in the last 4 minutes of test was analyzed off-line by an operator unaware of the pharmacological treatments.

\subsubsection{Open Field test}

One hour after the last treatment, animals were positioned in a corner of an openfield arena $(60 \mathrm{~cm} \times 70 \mathrm{~cm})$ and the general motor activity was assessed in a 5 minutes session. The covered distance $(\mathrm{cm})$ was evaluated using Smart 2.5 software.

\subsubsection{Neurochemical experiments}

\subsubsection{Western Blot analysis}

Male HDC-KO, PPAR- $\alpha-\mathrm{KO}$ and WT mice received i.p. injections of Vehicle, OEA $(10 \mathrm{mg} / \mathrm{kg})$ or imipramine (IMI) $(10 \mathrm{mg} / \mathrm{kg})$ daily for 8 days and $1 \mathrm{~h}$ later they were challenged in the TST, immediately after the test, they were sacrificed. The brains were dissected on ice, and the cortices and hippocampi isolated immediately. The cortices and the pooled hippocampi (left and right) were individually homogenized in $200 \mu \mathrm{L}$ ice-cold lysis buffer containing protease and phosphatase inhibitors (50mM TrisHCl pH 7.5, 50mM NaCl, 10 mM EGTA, 5mM EDTA, 2mM NaPP, 4 $\mathrm{mM}$ PNFF, $1 \mathrm{mM}$ Na3VO4, $1.1 \mathrm{mM}$ PMSF, 20 $\mu \mathrm{g} / \mu \mathrm{L}$ Leupeptin, $50 \mu \mathrm{g} / \mu \mathrm{L}$ Aprotinin, $0.1 \%$ SDS) and centrifuged at $12000 \mathrm{rpm}$ at $4{ }^{\circ} \mathrm{C}$ for 15 minutes. The supernatant was collected, and total protein levels were quantified using the Pierce BCA Protein Assay (Thermo Scientific, USA). Homogenates were diluted in a mix of lysis buffer and loading buffer 2x (50mM Tris pH 6.8, 100mM DTT, 10\% Glycerol, 1\% Bromophenol Blue and $2 \%$ SDS) and boiled for 10 minutes. Aliquots containing $40 \mu \mathrm{g}$ total proteins were resolved by electrophoresis on a $10 \%$ SDS-polyacrylamide gel (SDS-PAGE) and transferred onto polyvinylidene difluoride (PVDF) membranes (Immobilon Transfer Membranes, Millipore, USA). Blots were blocked in Tris-buffered saline, pH 7.6 containing $0.1 \%$ of Tween 20 (TBS-T) and 5\% non-fat dry milk (Bio-Rad Laboratories, USA) for $2 \mathrm{~h}$ at room temperature and then incubated overnight at $4{ }^{\circ} \mathrm{C}$ with monoclonal antibodies against $\alpha$ pospho-CREB (Ser 133) or $\alpha$ CREB (both from Cell Signaling Technology, USA) diluted 1:1000 in TBS-T containing 5\% bovine serum albumin or $5 \%$ non-fat dry milk, respectively. Immunodetection was performed with secondary antibodies (anti-rabbit IgG conjugated to horseradish peroxidase, Cell Signaling Technology, USA) diluted 1:5000 in TBS-T containing 1\% of non-fat dry milk. Membranes were washed in TBS-T and then reactive bands were detected using enhanced chemiluminescence (Luminata Crescendo, Millipore, USA). Quantitative densitometric analysis was performed using the QuantityOne analysis software (Bio$\mathrm{Rad})$. For each sample the ratio of $\alpha$ pSer133-CREB/ $\alpha \mathrm{CREB}$ densities was calculated and then all the individual rates were expressed as a percentage of the average of ratios obtained from control groups. 


\subsubsection{Statistical Analysis}

All values are expressed as means \pm SEM, and the number of mice used in each experiment is also indicated. The presence of significant treatment effects was determined by using a 1-way ANOVA followed by Newmann Keuls' MCT test, as appropriate. The level of significance was set at $\mathrm{P} \leq 0,05$.

\subsection{Results}

\subsubsection{Oleoylethanolamide systemic administration exerts antidepressant-like ef- fect by recruiting histaminergic neurotransmission}

We assessed the possible antidepressant-like effect of repeated or sub-chronic OEA treatment in the TST response of HDC-KO mice and their wild type littermates (WT). Imipramine was used a positive control.

Figure 10 shows the effect of different doses of OEA administered 3 times in 24 hrs. OEA increased immobility time in WT mice at both doses used, whereas it was ineffective in HDC-KO mice. A 2-way ANOVA revealed an overall significant difference between groups $\mathrm{F}_{\text {(genotype } x \text { treatment) 3,69 }}=5.026, \mathrm{p}<0.005$ ); $\mathrm{F}_{\text {(treatment) 3,69 }}=12.85$, $\mathrm{p}<0.0001 ; \mathrm{F}_{\text {(genotype) } 1,69}=4.446 \mathrm{p}<0.05$. Bonferonni's post hoc test showed that OEA treatment at both doses significantly decreased the immobility time of WT mice exposed to the TST $(5 \mathrm{mg} / \mathrm{kg}, \mathrm{p}<0.01 ; 10 \mathrm{mg} / \mathrm{kg}, \mathrm{p}<0.001)$, whereas no significant effects were observed in HDC-KO mice. Imipramine decreased the immobility time of both genotypes (WT and HDC-KO, p<0.001) (Figure 10A).

When tested after sub-chronic treatments ANOVA revealed an overall significant difference between HDC-KO and WT mice. Either OEA, imipramine or vehicle were administered i.p. for 8 consecutive days, and the last injection $1 \mathrm{hr}$ before the test a 2way ANOVA showed significant differences between groups ( $F_{\text {(genotype }} \mathrm{x}$ treatment) 3,57 $=$ $3.362, \mathrm{p}<0.05) ; \mathrm{F}_{\text {(treatment) } 3,57}=7.418, \mathrm{p}<0.001 ; \mathrm{F}_{\text {(genotype) } 1,57}=10.34 \mathrm{p}<0.01$ ). Bonferroni's post hoc analysis revealed that OEA treatment at all doses tested significantly reduced the immobility time of WT mice $(5 \mathrm{mg} / \mathrm{kg}$ and $10 \mathrm{mg} / \mathrm{kg} \mathrm{OEA,} \mathrm{p<0.001),}$ whereas no effect on immobility time was observed in HDC-KO mice. Imipramine reduced the immobility time of both genotypes $(p<0.001)$ (Figure 10B).

To avoid biases related to compensatory mechanisms due to chronic depletion of histamine, we assessed the effects of OEA in CD1 mice that received i.c.v. infusion of the HDC suicide inhibitor $\alpha$-FMHis. In analogy to the results observed in chronically histamine deprived mice, also in $\alpha$-FMHis-treated mice, ANOVA revealed an overall significant difference between groups $(\mathrm{F}(7,92)=14.35 ; \mathrm{P}<0,0001)$; In analogy to HDC-KO mice, OEA did not modify the immobility time of $\alpha$-FMHis treated mice $(97,4 \pm 25,0 \mathrm{~s})$ compared to vehicle-treated mice $(149,1 \pm 18,9 \mathrm{~s}$; Figure 12), whereas imipramine remained effective regardless of the presence of brain histamine $\left(\mathrm{F}_{\text {(icv } x \text { treatment) } 3,79}=4.707, \mathrm{p}<0.01\right) ; \mathrm{F}_{\text {(treatment) } 3,79}=43.62, \mathrm{p}<0.0001 ; \mathrm{F}_{\text {(icv) } 1,79}=5.007$, $\mathrm{p}<0.05)$. Significant differences for OEA treatments by Bonferroni's post hoc test were $\mathrm{p}<0.001$ (Figure 10C). 
Histaminergic neurotransmission as a gateway for the effects of Oleoylethanolamide

\subsubsection{OEA-induced increase in cortical and hippocampal CREB phosphoryla- tion is reduced in HDC-KO mice}

We compared the effect of OEA $(10 \mathrm{mg} / \mathrm{kg})$, imipramine $(10 \mathrm{mg} / \mathrm{kg})$ and vehicletreated $\mathrm{HDC}^{+/+}$and $\mathrm{HDC}^{-/-}$mice. Two-way ANOVA showed an overall significant difference between groups $\left(F_{\text {(genotype } x \text { treatment) } 2,21}=4.14, p<0.05 ; F_{\text {(treatment) } 2,21}=14.05\right.$, $\mathrm{p}=0.0001 ; \mathrm{F}_{\text {(genotype)1,21 }}=11.56, \mathrm{p}<0.05$ ). Bonferroni's post hoc test found significant differences between groups $(p<0.001$; Figure 11). OEA increased significantly pCREB in the hippocampus of $\mathrm{HDC}^{+/+}$mice compared with vehicle treated animals (Bonferroni's post hoc test $p<0.01$; Figure 11A). However, OEA treatment was ineffective in $\mathrm{HDC}^{-/-}$mice (Figure 11A), as pCREB levels were not different from those of vehicle treated mice. Similar to the effect in the hippocampus, OEA increased pCREB levels in the frontal cortex of $\mathrm{HDC}^{+/+}$mice (Figure 11B), but not in the cortex

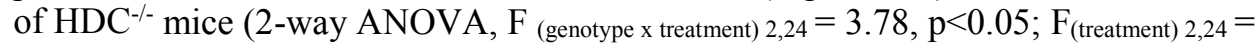
$6.78, \mathrm{p}<0.01 ; \mathrm{F}_{\text {(genotype) } 1,24}=3.57, \mathrm{p}=0.07$ ). Imipramine administered at $10 \mathrm{mg} / \mathrm{kg}$ for 8 days increased pCREB in the hippocampus and cortex of both genotypes with respect to controls $(\mathrm{p}<0.05)$.

\subsubsection{Oleoylethanolamide systemic administration reduced immobility time by targeting PPAR- $\alpha$}

To explore if PPAR- $\boldsymbol{\alpha}$ are required for the antidepressant-like effect of OEA, PPAR- $\boldsymbol{\alpha}$-KO mice and wild type littermates were tested in the TST after repeated or sub-chronic OEA $(10 \mathrm{mg} / \mathrm{kg})$ treatments as shown in Figure 12. A 2-way ANOVA was conducted to examine the effect of genotype and repeated ( $3 \mathrm{x}$ in $24 \mathrm{hrs})$ treatment on immobility time that revealed an overall significant difference between groups ( $\mathrm{F}$ (genotype $\mathrm{x}$ treatment) $1,29=5.924, \mathrm{P}<0.05 ; \mathrm{F}$ (treatment) $1,29=11.80, \mathrm{p}<0.01 ; \mathrm{F}_{\text {(genotype) } 1.29}=0.8$, $\mathrm{p}=0.3)$. Bonferroni post-hoc test showed that OEA treatment significantly decreased the immobility time of PPAR $-\alpha^{+/+}$mice $(p<0.001)$, but not of PPAR- $\alpha^{-/-}$mice (Figure $12 \mathrm{~A})$.

When tested after OEA sub-chronic treatments (daily injection for 8 days) ANOVA revealed an overall significant difference between groups ( $\mathrm{F}_{\text {(genotype } x} \mathrm{x}$ treatment)1,27 $=9.147 ; \mathrm{P}<0.01 ; \mathrm{F}$ (treatment)1,27 $=2.94, \mathrm{p}=0.09 ; \mathrm{F}_{\text {(genotype) } 1.27}=0.8, \mathrm{p}=0.28$ ). Post hoc analysis revealed a reduction in immobility time in WT mice treated with 10 $\mathrm{mg} / \mathrm{kg}$ OEA compared with VEH treated animals ( $<<0.01$;Figure 12B). In PPAR- $\alpha$ KO male mice no differences were observed between $10 \mathrm{mg} / \mathrm{kg}$ OEA and VEH treated animals (Figure 12B).

\subsubsection{OEA-induced increase in cortical and hippocampal CREB phosphoryla- tion is reduced in PPAR-alpha KO mice}

Figure 13 shows CREB phosphorylation at Ser 133 residue in cortical and hippocampal homogenates of normal and PPAR- $\alpha$ deficient mice treated sub-cronically with OEA $(10 \mathrm{mg} / \mathrm{kg})$ or vehicle. Two- way ANOVA revealed an overall difference in hippocampal $\left(\mathrm{F}_{\text {(genotype } x \text { treatment)1,30 }}=4.943, \mathrm{p}<0.05, \mathrm{~F}_{\text {(treatment) } 1,30}=10.61, \mathrm{p}<0.01\right.$; $\left.\mathrm{F}_{\text {(genotype) } 1,30}=0.6, \mathrm{p}=0.44\right)$ and cortical $\left(\mathrm{F}_{\text {(genotype } \mathrm{x} \text { treatment) } 1,26}=3.032, \mathrm{~F}_{\text {(treatment) } 1,26}=\right.$ 3.514, $\mathrm{p}=0.09 ; \mathrm{F}_{\text {(genotype) } 1,26}=0.02, \mathrm{p}=0.8$ ) homogenates between $\mathrm{WT}$ and PPAR $\alpha-\mathrm{KO}$ mice. Post-hoc comparisons showed a significant increase in pSer133CREB/CREB 
ratios after OEA treatment when compared with vehicle-treated control animals in both the hippocampus (Bonferroni's post hoc test $\mathrm{p}<0.01$; Figure 13A) and cortex (Bonferroni's post hoc test $p<0.05$; Figure 13B). No significant changes in CREB phosphorylation were observed in PPAR- $\alpha$ KO mice that received OEA or vehicle.

\subsubsection{General motility}

To exclude possible effects of the various treatments and genotypes on spontaneous locomotor activity that may have affect the immobility time in the TST, mice were exposed to the open field and motor activity recorded for $5 \mathrm{~min}$. As shown in figure 14 , no differences were observed between experimental groups.

\subsection{Summary of the Results (Part II)}

The present results indicated that repeated treatments with OEA reduced the immobility time in the tail suspension test when compared with vehicle treated $\mathrm{HDC}^{+/+}$ mice, but this effect is not observed in $\mathrm{HDC}^{-/-}$mice. All antidepressants effectively reduce immobility in this test (Lucki, 2001). Imipramine, a classic tricyclic antidepressant, reduced the immobility time in both genotypes. To understand if brain histamine is necessary for the behavioural effects of OEA, and to exclude compensatory mechanisms of $\mathrm{HDC}^{-/-}$mice, we also evaluated the behaviour in mice pharmacologically deprived of histamine by using an i.c.v injection of $\alpha-F M H$, a suicide inhibitor of HDC. Similarly to what we observed in $\mathrm{HDC}^{-/-}$mice, a significant reduction in the immobility time was observed in WT mice treated with OEA, but not in $\alpha$-FMHistreated mice. OEA treatments did not modify the exploratory behaviour in the open field test performed by each experimental group indicating that the effect observed in the TST is not related to alterations in the motor activity.

We tested the hypothesis that different levels of CREB phosphorylation - a transcription factor that serves as a convergence point for multiple classes of antidepressant drugs (Carlezon et al., 2005) - would explain the differences between genotypes in the observed behavioural tests. Our results also indicate that histamine signalling is necessary for OEA to trigger CREB phosphorylation. By using a sub-chronic treatment regimen, we observed a good correlation between behavioural and biochemical data. OEA and imipramine treatment reduced the immobility time and increased CREB phosphorylation in cortical and hippocampal homogenates of $\mathrm{HDC}^{+/+}$animals, whereas in $\mathrm{HDC}^{-/-}$mice OEA did not change immobility time, nor CREB phosphorylation levels. Furthermore, imipramine increased CREB phosphorylation and reduced immobility time independently of genotype. Our results mirror the observation of $\mathrm{Mu}-$ nari et al., (2015) who found that selective serotonin reuptake inhibitors (SSRI) such as citalopram reduced the immobility time and increased $\mathrm{pCREB}$ of $\mathrm{HDC}^{+/+}$mice, but was ineffective $\mathrm{HDC}^{-/-}$mice even though their serotonergic system is functional and the pCREB pathway is not compromised (Munari et al., 2015). Thus, our biochemical results indicate that the lack of CREB phosphorylation may contribute to OEA inefficacy in histamine-deficient mice.

Where histamine and OEA interact to exert their behavioural and neurochemical effects remains to be established. In this thesis, I provide evidence that OEA targets the PPAR- $\alpha$ to exerts its effects on the TST and CREB. To this end we evaluated the behaviour of PPAR- $\alpha^{+/+}$and PPAR- $\alpha^{-/-}$mice. We observed that repeated treatments 
Histaminergic neurotransmission as a gateway for the effects of Oleoylethanolamide

with OEA reduced the immobility time and increased pCREB in WT mice, but not in PPAR- $\alpha^{-/-}$mice.

In conclusion, our results confirm that OEA may improve animal depression-like behaviours as first observed by Jin et a., (2015) (Jin et al., 2015), but we unravelled a novel mechanism by which OEA achieves its effect, namely by recruiting the brain histaminergic system. We believe that activation of the histaminergic system, at least in the hippocampus and prefrontal cortex, is necessary for OEA-induced CREB phosphorylation and consequently the behavioural output.

Although OEA shows potential as an antidepressant that reverses cerebral functional abnormalities and ameliorates depression, further research should be performed on the specific pathways by which OEA affects the histaminergic system and behavioural responses. 


\section{Part III \\ Histaminergic involvement in Oleoylethanolamide protection on the cognitive decline induced by chronic social stress in mice}

\subsection{Materials and Methods}

\subsubsection{Animals and Drugs}

Male CD1 mice (9 weeks of age; Charles River, Italy), histidine decarboxylase knockout (HDC-KO) mice and wild type (WT) littermates (background c57B1/6), grown in the Centro Sabulazione Animali di Laboratorio (CeSAL), Università di Firenze were used for behavioural experiments. Genetically modified animals were routinely genotyped using the PCR protocol previously described (Provensi et al., 2014). Animals were housed in humidity and temperature-controlled room $\left(22-24{ }^{\circ} \mathrm{C}\right)$, allowed free access to food (4RF21; Mucedola s.r.1., Milan, Italy) and water, and kept on a 12-h light/dark cycle (lights start at 7:00 AM). All the experiments were conducted between 9:00 AM and 4:00 PM. OEA and vehicle were dissolved as previously reported (Result part I). The OEA or Vehicle treatments started at ten days before the end of stress procedure.

\subsubsection{Chronic Social Defeat Stress Paradigm}

Prior to the social defeat stress, we selected the aggressive CD1 resident. CD1 mice were screened for aggressive behavior: latency of first attack was monitored and dominance status of mice was visually determined. Mice were deemed dominant if they displayed aggressive behavior toward their opponent (another CD1) such as tail rattling, chasing, biting and fight-attacks. Mice were submissive if they displayed defending and avoidance behaviour such as escaping, defensive response, upright posture and defensive immobility. Mice with latencies to attack of $>30 \mathrm{~s}$ were not selected. The selected mice were those that were the most aggressive, most dominant, the heaviest and those which had the lowest latency to attack, for two consecutive days.

Chronic Social Defeat Stress (CSDS), was a 21-days social defeat adapted from previous studies (Bartolomucci et al., 2001; Keeney et al., 2006). In this paradigm the $\mathrm{C} 57 \mathrm{Bl} / 6$ mice were daily introduced in the cage of CD1 aggressive resident until the first attack. A 3-min cut-off for latency of attack was imposed in order to maintain the interaction between mice short. Thereafter, mice were physically separated by a perforated transparent divider and remained in sensory (olfactory and visual) contact for $2 \mathrm{~h}$. After that the divider is removed and the second attack occur. Stresses mice were 
Histaminergic neurotransmission as a gateway for the effects of Oleoylethanolamide

single housed during the entire period of stress. Control mice were left undisturbed housed in their own home cage with other control mouse. This chronic stress was combined with overcrowding conditions because housing density affects rodent behaviour (Beery and Kaufer, 2015) and crowded social environments have been used as stressors in the protocol. Food consumption and body weight were evaluated daily during the protocol.

\subsubsection{Social Interaction Test}

The social interaction test is used to assess social behaviour (Berton and Nestler, 2006). This is a two-step procedure. In the first $2.5 \mathrm{~min}$ session, the experimental $\mathrm{C} 57 \mathrm{BL} / 6$ mouse is allowed to freely explore an open field arena $(41 \mathrm{~cm}$ length $\times 32 \mathrm{~cm}$ width $\times 15 \mathrm{~cm}$ height). Along one side of the arena is a rectangular (9.5 cm length $\times 7.5 \mathrm{~cm}$ width $\times 5 \mathrm{~cm}$ height) wire cage that remains empty during the first trial (target absent condition). The C57BL/6 mouse is then removed from the testing arena for 1 minute (into a home cage), and a novel CD1 male mouse is placed into the wire cage. In the second $2.5 \mathrm{~min}$ trial (target present condition), the experimental C57BL/6 mouse is reintroduced into this arena now containing a social target (unfamiliar CD1 mouse) within the rectangular wire cage. Time (sec) spent in the interaction zone (surrounding wire cage) in the presence of the social target (Iñiguez et al., 2014), served as dependent variables.

\subsubsection{Novel object recognition test}

Object recognition paradigm measures a form of memory based on short and unrepeated experiments without any reinforcement, such as food or electric shocks (Ennaceur and Delacour, 1988). Object recognition is a one-trial task, and does not involve the learning of any rule, being entirely based on the spontaneous exploratory behavior of rodents toward objects. Mice were placed in a white polyvinylchloride box $(70 \times 60 \mathrm{~cm}$ and $30 \mathrm{~cm}$ high) with a grid floor that is easily cleaned and illuminated by a $75-\mathrm{W}$ lamp suspended $50 \mathrm{~cm}$ above the box. The objects to be discriminated were grey polyvinyl shapes: cubes of $8 \mathrm{~cm}$ side, pyramids and cylinders of $8 \mathrm{~cm}$ height. The object recognition task consisted of a training phase (T1) and a testing phase (T2). Twenty-four h prior to T1, they were habituated for two 10 min-session to the experimental apparatus in the absence of any object. Each mouse was subjected to the procedure separately and care was taken to remove any olfactory/taste cues by cleaning carefully the arena and test objects between trials. On the day of the experiment, the mouse was placed for $5 \mathrm{~min}$ into the test arena facing the same direction and in the same position in the presence of two identical plastic objects (cubes) (T1). The behaviour of mice was videotaped, and the time spent actively exploring the objects was measured. Exploration was defined as sniffing or touching the objects with the nose and/or forepaws. Sitting on or turning around the objects was not considered exploratory behaviour. T2 was performed $1 \mathrm{~h}$ after T1, during which, each mouse was again placed in the test arena for $5 \mathrm{~min}$ in the presence of one of the familiar objects and a novel object. The position of the objects (left/right) was randomized to prevent bias from order or place preference. Mice were placed in their home cage between trials. The behaviour of mice during T2 was videotaped, and the exploration periods of the familiar $(\mathrm{F})$ and the new object $(\mathrm{N})$ were measured. Care was taken to avoid 
place preference and olfactory stimuli by randomly changing the role (familiar or new object), and the position of the two objects during T2, and cleaning them carefully. Object recognition was carried out in an insulated room to avoid any noise that could impair the performance of the mouse.

\subsubsection{Statistical analysis}

All values are expressed as means \pm SEM, and the number of mice used in each experiment is also indicated. The presence of significant treatment effects was determined by a 2-way ANOVA followed by Bonferroni MCT test, as appropriate. The level of significance was set at $\mathrm{P} \leq 0,05$. Statistical analysis was performed using GraphPad Software. The data of the object recognition test are expressed as a percentage of time spent exploring each object during T1 and T2.

\subsection{Results (Part III)}

\subsubsection{Chronic Social Defeat Stress induced body weight gain and increased food consumption in OEA or VEH treated mice compared to controls}

Figure 15 shows the effect of OEA $(10 \mathrm{mg} / \mathrm{kg})$ or vehicle i.p. injections in mice submitted to the chronic social defeat stress (CSDS) on body weight and food consumption. Two-way ANOVA revealed no statistical significance between groups in WT mice (Figure 15A) nor in the HDC-KO mice (Figure 15B). Figure 15C shows the cumulative food consumption of WT mice treated with OEA or VEH and subjected to the CSDS protocol. Two-way ANOVA revealed no statistical significance between groups. Figure 15D shows the cumulative food consumption of HDC-KO mice treated with OEA or VEH and subjected to the CSDS protocol. Two-way ANOVA revealed an overall significant difference between groups ( $F_{\text {(treat- }}$ ments)2,336 $\left.=198.9, \mathrm{p}<0.0001 ; \mathrm{F}_{(\text {days }) 20,336}=952.76, \mathrm{p}<0.0001\right)$.

\subsubsection{Oleoylethanolamide reduces social avoidance induced by social defeat stress}

Figure 16 shows the effect of OEA systemic administration to stressed animals submitted to CSDS compared to controls, in the social avoidance test. OEA was administered for the 10 days preceding the test. One-way ANOVA revealed an overall significant difference between groups $(\mathrm{F} 2,17=20.36 \quad \# \mathrm{p}<0.05$ OEA vs VEH; $* * \mathrm{p}<0.001$ OEA vs NON-STRESSED; $* * * \mathrm{p}>0.0001$ VEH vs NON-STRESSED). Stressed mice spent less time in the interaction with the CD1 mouse compared to nonstressed control mice, therefore, time spent interacting was influenced by stress exposure. OEA treatment increased the interaction time when compared to controls treated with vehicle (Figure 16A). On the contrary, ANOVA revealed no significant differences among HDC-KO regardless of the treatment either (OEA or vehicle). However, an overall significant difference was revealed between stressed and non-stressed groups (1-way ANOVA and Bonferroni post-hoc test; F2,18=10.23 $* \mathrm{P}<0.05$ $* * \mathrm{p}<0.001$; Figure 16B). 
Histaminergic neurotransmission as a gateway for the effects of Oleoylethanolamide

\subsubsection{Oleoylethanolamide improves the performance in the object recognition test of WT stressed mice, but not of HDC-KO mice}

Figure 17 shows the effect of OEA administration on mice performances in the object recognition test. No significant group effects were detected during T1 (twoway ANOVA and Bonferroni post-hoc test) (Figure 17A). During T2, given 1 hour after the T1, Two-way ANOVA revealed an overall difference between groups ( $\mathrm{F}$ (objects $x$ treatment)2,38 $=1.915 \mathrm{P}=0.16 ; \mathrm{F}$ (objects) $1,38=18.10, \mathrm{p}<0.0001 ; \mathrm{F}$ (treatment)2,38 $=6.330 \mathrm{e}$ 014, $\mathrm{p}=1)$. Non-stressed control mice spent more time exploring the familiar object $(\mathrm{p}<0.001)$. Similarly, stressed mice treated with OEA spent significantly more time exploring the new object than the familiar one $(p<0.01)$. On the contrary, 2-way ANOVA revealed no significant differences in the exploration of the two objects of stressed mice treated with vehicle (Figure 17B). In HDC-KO mice no significant group effects were detected during T1 (2way ANOVA and Bonferroni post-hoc test) (Figure 17C). During T2 Two-way ANOVA revealed an overall statistical difference between groups $(\mathrm{F}$ (objects $x$ treatment)2,31 $=4.536, \mathrm{P}<0.01 ; \mathrm{F}$ (objects)1,31 $=0.14, \mathrm{p}=0.7 ; \mathrm{F}$ (treatment)2,31 $=0.005, p=0.99)$. Non-stressed HDC-KO mice spent more time in the exploration of the novel object compared to familiar one $(p<0.05)$. No differences in the exploration of the familiar and the novel object were observed in stressed HDC-KO mice treated with OEA or Vehicle (2way ANOVA and Bonferroni post-hoc test) (Figure 17D).

\subsubsection{CSDS did not affect motility of mice tested in the open field}

When evaluated in the open field test ANOVA revealed no significant differences between experimental groups after treatments with OEA or VEH in stressed WT mice compared to controls (Figure 18). Similarly, HDC-KO mice motility was not affected by stress or treatments.

\subsection{Summary of the Results (Part III)}

Stress and traumatic events are increasingly recognized as risk factors for mental disorders in particular for depression, anxiety disorders, and post-traumatic stress disorder (Musazzi et al., 2017; Selten and Cantor-Graae, 2005, 2007; Selten et al., 2013) (PTSD). Several rodent models of neuropsychiatric disorders use chronic stress, measuring behavioural and neurochemical readouts at the end of the protocols.

The present data indicate that the social defeat stress induce social avoidance behaviour as observed by reduction of interaction time in the social interaction test between stressed and non stressed mice. It has been widely reported that defeated animals of different species spend on average significantly less time in close proximity to a social target in a social interaction test as compared to non-defeated animals (Berton and Nestler, 2006; Dadomo et al., 2011; Hollis et al., 2010). The treatment with OEA reduce the social avoidance in WT compared to vehicle treated mice and non stressed controls. To understand if brain histamine is necessary for the behavioural effects of OEA, we also evaluated the behaviour in HDC-KO mice. The OEA effect in reducing social avoidance was not observed in histamine deprived animals.

Stress is a biologically significant factor that, by altering brain cell properties, can disturb cognitive processes such as learning and memory (Kim and Diamond, 


\section{Alessia Costa}

2002). In this respect, we showed here that chronic social defeat stress causes memory impairment in stressed animals when tested in the object recognition test. Interestingly treatment with OEA was able to prevent this deficit, and again histamine was involved also in this effect since improvement in recognition memory was not observed in histamine-deficient animals. No relevant changes in body weight or food consumption were observed in all groups. Therefore, the integrity of central histaminergic system is essential for the OEA to induce beneficial effects on stress-related responses. 



\section{Conclusions and Discussion}

The histamine system is a pleiotropic system. Histamine plays a major role in the regulation of autonomic functions, including energy balance, sleep and regulation of body temperature (Haas and Sergeeva, 2012) and is crucial in controlling arousal and cognition (Köhler et al., 2011). HA-synthesizing neurons are strongly conserved throughout vertebrate phylogeny, from zebrafish to humans reflecting the important roles they play in many aspects of physiology and behavior (Haas and Panula, 2003; Haas et al., 2008; Panula and Nuutinen, 2013; Passani et al., 2007; Schwartz et al., 1991). In mammals, histaminergic neurons are only found in the tuberomamillary nucleus (TMN) of the posterior hypothalamus (Panula et al., 1984; Watanabe et al., 1984) and extend a widespread and diffuse, network of unmyelinated fibers throughout the central nervous system, including interconnections with other arousal-related neuromodulatory systems (Panula and Nuutinen, 2013). Disruptions of histaminergic neurotransmission have been implicated in a variety of neuropsychiatric disorders (Baronio et al., 2014; Shan et al., 2015a; Shan et al., 2015b).

The histamine system has been suggested as a possible target for the treatment of psychiatric disorders, and drugs that modulate this system have been especially proposed as cognitive enhancers (Passani and Blandina, 2011; Tiligada et al., 2011). Therefore, a better understanding of the histamine system would be of great use for the development of new, much needed, pharmacological treatments for psychiatric disorders.

Oleoylethanolamide is an endogenous lipid mediator that inhibits feeding and in rodents, intestinal OEA levels increase about threefold upon refeeding, a response that may contribute to the induction of between-meal satiety (Piomelli, 2013). OEA was also identified in the gastrointestinal tract of Python molurus (Astarita et al., 2006) suggesting that this lipid messenger may be widespread among vertebrate groups and may represent an evolutionarily ancient means of regulating energy intake.

It was recently demonstrated in our laboratory that brain histamine mediates the central effects of a signal molecule produced in the intestine, namely oleoylethanolamide (OEA). OEA is released by the enterocytes in response to high fat intake and reduces eating (Fu et al., 2005), by indirectly activating a subpopulation of histaminergic neurons (Provensi et al., 2014).

In my thesis, I explored other potential interactions between these two phylogenetically ancient systems in the pursuit of unexplored neuronal mechanisms that may shed light on the mode of action of psychoactive agents and possibly lead to the development of new drugs. 
Histaminergic neurotransmission as a gateway for the effects of Oleoylethanolamide

OEA was shown to affect not only feeding behaviour, but also the consolidation of aversive memories (Campolongo et al., 2009). Here, I demonstrated that the activation of the histaminergic system in the amygdala of rats is necessary for OEA to modulate the consolidation of fear memories (Provensi et al., 2017).

As reported in the present thesis we also demonstrated that OEA has a potential antidepressant effect since it reduces immobility time in the TST, a paradigm widely used to predict antidepressant properties of several compounds. The reduction of immobility time occurred in normal but not in histamine-deprived mice. In a parallel fashion, OEA, as other antidepressant compounds elicited CREB phosphorylation in normal mice but not in brain histamine-deprived mice, nor in PPAR- $\alpha^{-/-}$mice.

Hence, we provided evidence that the molecular target for the antidepressant-like of OEA is PPAR- $\alpha$. The most conservative hypothesis is that activation of peripheral PPAR- $\alpha$ in turn recruit the histaminergic system in a yet unexplored fashion. Understanding the mechanisms of OEA's antidepressant-like action could improve the understanding of depression and the treatment of a disease that is not completely understood and do not respond adequately to available agents.

The several roles of OEA in brain disorders led us to test its effects also in stressrelated disorders. Stress, as previously described, is known to induce physical, behavioural and neuropathological outcomes. Here we showed that the maladaptive behavioural responses to chronic stress, such as memory impairments and a depression-like phenotype may be prevented by OEA sub-chronic treatment. Once again, we evaluated the role histaminergic system play in such effect, considering its involvement in stress responses (Ito, 2000). Here, I reported that the beneficial effect of OEA to prevent stress-induced behavioural impairments are only achieved in mice with an intact brain histamine system. Therefore, in this paradigm as well, brain histamine is necessary for OEA's neurological effect.

Our current working hypothesis is based on the concept that histaminergic neurons act as a relay station that integrate peripheral signals, organize them into a coherent output to selected brain regions hence sending information to other brain areas in order to create a neuro-circuitry responsible for central OEA's effects.

OEA could be just an example, but many other peripheral signals may influence brain functions using this mechanism. This hypothesis implies that histamine neurons in the TMN are organized in distinct subpopulations differently regulated and innervating specific brain areas to allow multiple processes.

The identification of signalling pathways connecting the periphery with the central nervous system is fascinating and holds great promise for the development of new therapeutics, as targeting peripheral systems may provide a more easily accessible therapeutic strategy for pharmaceutical treatment. 


\section{References}

Adler, N. E., Boyce, T., Chesney, M. A., Cohen, S., Folkman, S., Kahn, R. L., Syme, S. L., 1994. Socioeconomic status and health. The challenge of the gradient. Am Psychol 49, 15-24.

Aggleton, J. P., Brown, M. W., 2006. Interleaving brain systems for episodic and recognition memory. Trends Cogn Sci 10, 455-463.

Airaksinen, M. S., Paetau, A., Paljärvi, L., Reinikainen, K., Riekkinen, P., Suomalainen, R., Panula, P., 1991a. Histamine neurons in human hypothalamus: anatomy in normal and Alzheimer diseased brains. Neuroscience 44, 465-481.

Airaksinen, M. S., Reinikainen, K., Riekkinen, P., Panula, P., 1991b. Neurofibrillary tangles and histamine-containing neurons in Alzheimer hypothalamus. Agents Actions 33, 104-107.

Alvarez, E. O., Banzan, A. M., 1996. Hippocampus and learning: possible role of histamine receptors. Medicina (B Aires) 56, 155-160.

Alvarez, E. O., Banzan, A. M., 2001. Functional regional distribution of histamine receptors in the rat hippocampus: modulation of learning of an active avoidance response. J Neural Transm (Vienna) 108, 1249-1261.

Alvarez, E. O., Banzan, A. M., 2008. The activation of histamine-sensitive sites of the ventral hippocampus modulates the consolidation of a learned active avoidance response in rats. Behav Brain Res 189, 92-99.

Amat, J., Aleksejev, R. M., Paul, E., Watkins, L. R., Maier, S. F., 2010. Behavioral control over shock blocks behavioral and neurochemical effects of later social defeat. Neuroscience 165, 1031-1038.

Angeles-Castellanos, M., Aguilar-Roblero, R., Escobar, C., 2004. c-Fos expression in hypothalamic nuclei of food-entrained rats. Am J Physiol Regul Integr Comp Physiol 286, R158-165.

Anichtchik, O. V., Huotari, M., Peitsaro, N., Haycock, J. W., Männistö, P. T., Panula, P., 2000. Modulation of histamine $\mathrm{H} 3$ receptors in the brain of 6-hydroxydopamine-lesioned rats. Eur J Neurosci 12, 3823-3832.

Anisman, H., Grimmer, L., Irwin, J., Remington, G., Sklar, L. S., 1979. Escape performance after inescapable shock in selectively bred lines of mice: response maintenance and catecholamine activity. J Comp Physiol Psychol 93, 229-241.

Arrang, J. M., Morisset, S., Gbahou, F., 2007. Constitutive activity of the histamine H3 receptor. Trends Pharmacol Sci 28, 350-357. 
Histaminergic neurotransmission as a gateway for the effects of Oleoylethanolamide

Astarita, G., Rourke, B. C., Andersen, J. B., Fu, J., Kim, J. H., Bennett, A. F., Hicks, J. W., Piomelli, D., 2006. Postprandial increase of oleoylethanolamide mobilization in small intestine of the Burmese python (Python molurus). Am J Physiol Regul Integr Comp Physiol 290, R1407-1412.

BACHUR, N. R., MASEK, K., MELMON, K. L., UDENFRIEND, S., 1965. FATTY ACID AMIDES OF ETHANOLAMINE IN MAMMALIAN TISSUES. J Biol Chem 240, 1019-1024.

Bai, F., Li, X., Clay, M., Lindstrom, T., Skolnick, P., 2001. Intra- and interstrain differences in models of "behavioral despair". Pharmacol Biochem Behav 70, 187-192.

Bailey, M. T., Coe, C. L., 1999. Maternal separation disrupts the integrity of the intestinal microflora in infant rhesus monkeys. Dev Psychobiol 35, 146-155.

Baldan, L. C., Williams, K. A., Gallezot, J. D., Pogorelov, V., Rapanelli, M., Crowley, M., Anderson, G. M., Loring, E., Gorczyca, R., Billingslea, E., Wasylink, S., Panza, K. E., Ercan-Sencicek, A. G., Krusong, K., Leventhal, B. L., Ohtsu, H., Bloch, M. H., Hughes, Z. A., Krystal, J. H., Mayes, L., de Araujo, I., Ding, Y. S., State, M. W., Pittenger, C., 2014. Histidine decarboxylase deficiency causes tourette syndrome: parallel findings in humans and mice. Neuron 81, 77-90.

Baldi, E., Bucherelli, C., 2005. The inverted "u-shaped" dose-effect relationships in learning and memory: modulation of arousal and consolidation. Nonlinearity Biol Toxicol Med 3, 9-21.

Baldi, E., Lorenzini, C. A., Bucherelli, C., 2004. Footshock intensity and generalization in contextual and auditory-cued fear conditioning in the rat. Neurobiol Learn Mem 81, 162-166.

Barker, G. R., Bird, F., Alexander, V., Warburton, E. C., 2007. Recognition memory for objects, place, and temporal order: a disconnection analysis of the role of the medial prefrontal cortex and perirhinal cortex. J Neurosci 27, 2948-2957.

Baronio, D., Gonchoroski, T., Castro, K., Zanatta, G., Gottfried, C., Riesgo, R., 2014. Histaminergic system in brain disorders: lessons from the translational approach and future perspectives. Ann Gen Psychiatry 13, 34.

Barrot, M., Olivier, J. D., Perrotti, L. I., DiLeone, R. J., Berton, O., Eisch, A. J., Impey, S., Storm, D. R., Neve, R. L., Yin, J. C., Zachariou, V., Nestler, E. J., 2002. CREB activity in the nucleus accumbens shell controls gating of behavioral responses to emotional stimuli. Proc Natl Acad Sci U S A 99, 11435-11440.

Bartolomucci, A., Palanza, P., Gaspani, L., Limiroli, E., Panerai, A. E., Ceresini, G., Poli, M. D., Parmigiani, S., 2001. Social status in mice: behavioral, endocrine and immune changes are context dependent. Physiol Behav 73, 401-410.

Bealer, S. L., 1993. Histamine releases norepinephrine in the paraventricular nucleus/anterior hypothalamus of the conscious rat. J Pharmacol Exp Ther 264, 734-738.

Bealer, S. L., Abell, S. O., 1995. Paraventricular nucleus histamine increases blood pressure by adrenoreceptor stimulation of vasopressin release. Am J Physiol 269, H80-85.

Beery, A. K., Kaufer, D., 2015. Stress, social behavior, and resilience: insights from rodents. Neurobiol Stress 1, 116-127.

Belmaker, R. H., Agam, G., 2008. Major depressive disorder. N Engl J Med 358, 55-68.

Benarroch, E. E., 2010. Histamine in the CNS: multiple functions and potential neurologic implications. Neurology 75, 1472-1479. 
Benetti, F., Baldi, E., Bucherelli, C., Blandina, P., Passani, M. B., 2013. Histaminergic ligands injected into the nucleus basalis magnocellularis differentially affect fear conditioning consolidation. Int J Neuropsychopharmacol 16, 575-582.

Benetti, F., da Silveira, C. K., da Silva, W. C., Cammarota, M., Izquierdo, I., 2012. Histamine reverses a memory deficit induced in rats by early postnatal maternal deprivation. Neurobiol Learn Mem 97, 54-58.

Benetti, F., Furini, C. R., de Carvalho Myskiw, J., Provensi, G., Passani, M. B., Baldi, E., Bucherelli, C., Munari, L., Izquierdo, I., Blandina, P., 2015. Histamine in the basolateral amygdala promotes inhibitory avoidance learning independently of hippocampus. Proc Natl Acad Sci U S A 112, E2536-2542.

Benetti, F., Izquierdo, I., 2013. Histamine infused into basolateral amygdala enhances memory consolidation of inhibitory avoidance. Int J Neuropsychopharmacol 16, 1539-1545.

Bennett, C. T., Pert, A., 1974. Antidiuresis produced by injections of histamine into the cat supraoptic nucleus. Brain Res 78, 151-156.

Bercik, P., 2011. The microbiota-gut-brain axis: learning from intestinal bacteria? Gut 60, 288-289.

Bercik, P., Park, A. J., Sinclair, D., Khoshdel, A., Lu, J., Huang, X., Deng, Y., Blennerhassett, P. A., Fahnestock, M., Moine, D., Berger, B., Huizinga, J. D., Kunze, W., McLean, P. G., Bergonzelli, G. E., Collins, S. M., Verdu, E. F., 2011. The anxiolytic effect of Bifidobacterium longum NCC3001 involves vagal pathways for gut-brain communication. Neurogastroenterol Motil 23, 1132-1139.

Bernabeu, R., Cammarota, M., Izquierdo, I., Medina, J. H., 1997. Involvement of hippocampal AMPA glutamate receptor changes and the cAMP/protein kinase A/CREB-P signalling pathway in memory consolidation of an avoidance task in rats. Braz J Med Biol Res 30, 961-965.

Berthoud, H. R., Morrison, C., 2008. The brain, appetite, and obesity. Annu Rev Psychol 59, 55-92.

Berton, O., Nestler, E. J., 2006. New approaches to antidepressant drug discovery: beyond monoamines. Nat Rev Neurosci 7, 137-151.

Beylin, A. V., Shors, T. J., 1998. Stress enhances excitatory trace eyeblink conditioning and opposes acquisition of inhibitory conditioning. Behav Neurosci 112, 1327-1338.

Bhargava, K. P., Kulshrestha, V. K., Santhakumari, G., Srivastava, Y. P., 1973. Mechanism of histamine-induced antidiuretic response. Br J Pharmacol 47, 700-706.

Bilbao, A., Blanco, E., Luque-Rojas, M. J., Suárez, J., Palomino, A., Vida, M., Araos, P., Bermúdez-Silva, F. J., Fernández-Espejo, E., Spanagel, R., Rodríguez de Fonseca, F., 2013. Oleoylethanolamide dose-dependently attenuates cocaine-induced behaviours through a PPAR $\alpha$ receptor-independent mechanism. Addict Biol 18, 78-87.

Binder, S., Dere, E., Zlomuzica, A., 2015. A critical appraisal of the what-where-when episodic-like memory test in rodents: Achievements, caveats and future directions. Prog Neurobiol 130, 71-85.

Blandina, P., Munari, L., Provensi, G., Passani, M. B., 2012. Histamine neurons in the tuberomamillary nucleus: a whole center or distinct subpopulations? Front Syst Neurosci 6,33 . 
Histaminergic neurotransmission as a gateway for the effects of Oleoylethanolamide

Bocchio-Chiavetto, L., Bagnardi, V., Zanardini, R., Molteni, R., Nielsen, M. G., Placentino, A., Giovannini, C., Rillosi, L., Ventriglia, M., Riva, M. A., Gennarelli, M., 2010. Serum and plasma BDNF levels in major depression: a replication study and meta-analyses. World J Biol Psychiatry 11, 763-773.

Bohus, B., de Kloet, E., Veldhuis, H., 1982. Adrenal Steroids and Behavioral Adaptation: Relationship to Brain Corticoid Receptors. Springer-Verlag.

Bowman, R. E., Beck, K. D., Luine, V. N., 2003. Chronic stress effects on memory: sex differences in performance and monoaminergic activity. Horm Behav 43, 48-59.

Bravo, J. A., Forsythe, P., Chew, M. V., Escaravage, E., Savignac, H. M., Dinan, T. G., Bienenstock, J., Cryan, J. F., 2011. Ingestion of Lactobacillus strain regulates emotional behavior and central GABA receptor expression in a mouse via the vagus nerve. Proc Natl Acad Sci U S A 108, 16050-16055.

Briley, M., Moret, C., 2000. Present and future anxiolytics. IDrugs 3, 695-699.

Brown, M. W., Banks, P. J., 2015. In search of a recognition memory engram. Neurosci Biobehav Rev 50, 12-28.

Brown, M. W., Warburton, E. C., Aggleton, J. P., 2010. Recognition memory: material, processes, and substrates. Hippocampus 20, 1228-1244.

Brown, R. E., Reymann, K. G., 1996. Histamine H3 receptor-mediated depression of synaptic transmission in the dentate gyrus of the rat in vitro. J Physiol 496 ( Pt 1), 175-184.

Brown, S. M., Henning, S., Wellman, C. L., 2005. Mild, short-term stress alters dendritic morphology in rat medial prefrontal cortex. Cereb Cortex 15, 1714-1722.

Brown, V., Ennis, M., 2001. Flow-cytometric analysis of basophil activation: inhibition by histamine at conventional and homeopathic concentrations. Inflamm Res 50 Suppl 2, S47-48.

BUSER, P., ROUGEUL, A., 1961. Somatic functions of the nervous system. Annu Rev Physiol 23, 387-418.

Buwalda, B., Kole, M. H., Veenema, A. H., Huininga, M., de Boer, S. F., Korte, S. M., Koolhaas, J. M., 2005. Long-term effects of social stress on brain and behavior: a focus on hippocampal functioning. Neurosci Biobehav Rev 29, 83-97.

Cahill, L., McGaugh, J. L., 1998. Mechanisms of emotional arousal and lasting declarative memory. Trends Neurosci 21, 294-299.

Campolongo, P., Roozendaal, B., Trezza, V., Cuomo, V., Astarita, G., Fu, J., McGaugh, J. L., Piomelli, D., 2009. Fat-induced satiety factor oleoylethanolamide enhances memory consolidation. Proc Natl Acad Sci U S A 106, 8027-8031.

Carlezon, W. A., Duman, R. S., Nestler, E. J., 2005. The many faces of CREB. Trends Neurosci $28,436-445$.

Carmichael, S. T., Price, J. L., 1995. Limbic connections of the orbital and medial prefrontal cortex in macaque monkeys. J Comp Neurol 363, 615-641.

Charney, D. S., 2004. Psychobiological mechanisms of resilience and vulnerability: implications for successful adaptation to extreme stress. Am J Psychiatry 161, 195-216.

Chen, A. C., Shirayama, Y., Shin, K. H., Neve, R. L., Duman, R. S., 2001. Expression of the cAMP response element binding protein (CREB) in hippocampus produces an antidepressant effect. Biol Psychiatry 49, 753-762. 
Chen, Z. Y., Jing, D., Bath, K. G., Ieraci, A., Khan, T., Siao, C. J., Herrera, D. G., Toth, M., Yang, C., McEwen, B. S., Hempstead, B. L., Lee, F. S., 2006. Genetic variant BDNF (Val66Met) polymorphism alters anxiety-related behavior. Science 314, 140-143.

Chiba, A. A., Kesner, R. P., Gibson, C. J., 1997. Memory for temporal order of new and familiar spatial location sequences: role of the medial prefrontal cortex. Learn Mem 4, 311-317.

Christian, J. J., 1971. Population density and reproductive efficiency. Biol Reprod 4, 248-294.

CHRISTIAN, J. J., LEMUNYAN, C. D., 1958. Adverse effects of crowding on lactation and reproduction of mice and two generations of their progeny. Endocrinology 63, 517-529.

Chudasama, Y., Robbins, T. W., 2003. Dissociable contributions of the orbitofrontal and infralimbic cortex to pavlovian autoshaping and discrimination reversal learning: further evidence for the functional heterogeneity of the rodent frontal cortex. J Neurosci 23, 8771-8780.

Clark, R. A., Gallin, J. I., Kaplan, A. P., 1975. The selective eosinophil chemotactic activity of histamine. J Exp Med 142, 1462-1476.

Clark, W. G., Cumby, H. R., 1976. Biphasic changes in body temperature produced by intracerebroventricular injections of histamine in the cat. J Physiol 261, 235-253.

Cohn, C. K., Ball, G. G., Hirsch, J., 1973. Histamine: effect on self-stimulation. Science 180, 757-758.

Conrad, C. D., 2005. THE RELATIONSHIP BETWEEN ACUTE GLUCOCORTICOID LEVELS AND HIPPOCAMPAL FUNCTION DEPENDS UPON TASK AVERSIVENESS AND MEMORY PROCESSING STAGE. Nonlinearity Biol Toxicol Med 3, 57-78.

Conti, A. C., Cryan, J. F., Dalvi, A., Lucki, I., Blendy, J. A., 2002. cAMP response elementbinding protein is essential for the upregulation of brain-derived neurotrophic factor transcription, but not the behavioral or endocrine responses to antidepressant drugs. J Neurosci 22, 3262-3268.

Cordero, M. I., Merino, J. J., Sandi, C., 1998. Correlational relationship between shock intensity and corticosterone secretion on the establishment and subsequent expression of contextual fear conditioning. Behav Neurosci 112, 885-891.

Cote, N. K., Harrington, M. E., 1993. Histamine phase shifts the circadian clock in a manner similar to light. Brain Res 613, 149-151.

Covington, H. E., Vialou, V., Nestler, E. J., 2010. From synapse to nucleus: novel targets for treating depression. Neuropharmacology 58, 683-693.

Cryan, J. F., Markou, A., Lucki, I., 2002. Assessing antidepressant activity in rodents: recent developments and future needs. Trends Pharmacol Sci 23, 238-245.

Cryan, J. F., Mombereau, C., 2004. In search of a depressed mouse: utility of models for studying depression-related behavior in genetically modified mice. Mol Psychiatry 9, 326-357.

Cryan, J. F., Mombereau, C., Vassout, A., 2005. The tail suspension test as a model for assessing antidepressant activity: review of pharmacological and genetic studies in mice. Neurosci Biobehav Rev 29, 571-625. 
Histaminergic neurotransmission as a gateway for the effects of Oleoylethanolamide

Cryan, J. F., O’Mahony, S. M., 2011. The microbiome-gut-brain axis: from bowel to behavior. Neurogastroenterol Motil 23, 187-192.

Curzon , P., Rustay, N., Browman, K., 2009. Cued and Contextual Fear Conditioning for Rodents. Boca Raton (FL): CRC Press/Taylor \& Francis Frontiers in Neuroscience.

Czéh, B., Michaelis, T., Watanabe, T., Frahm, J., de Biurrun, G., van Kampen, M., Bartolomucci, A., Fuchs, E., 2001. Stress-induced changes in cerebral metabolites, hippocampal volume, and cell proliferation are prevented by antidepressant treatment with tianeptine. Proc Natl Acad Sci U S A 98, 12796-12801.

Cámara, R. J., Ziegler, R., Begré, S., Schoepfer, A. M., von Känel, R., group, S. I. B. D. C. S. S., 2009. The role of psychological stress in inflammatory bowel disease: quality assessment of methods of 18 prospective studies and suggestions for future research. Digestion $80,129-139$.

da Silva, W. C., Bonini, J. S., Bevilaqua, L. R., Izquierdo, I., Cammarota, M., 2006. Histamine enhances inhibitory avoidance memory consolidation through a $\mathrm{H} 2$ receptor-dependent mechanism. Neurobiol Learn Mem 86, 100-106.

Dadomo, H., Sanghez, V., Di Cristo, L., Lori, A., Ceresini, G., Malinge, I., Parmigiani, S., Palanza, P., Sheardown, M., Bartolomucci, A., 2011. Vulnerability to chronic subordination stress-induced depression-like disorders in adult $129 \mathrm{SvEv}$ male mice. Prog Neuropsychopharmacol Biol Psychiatry 35, 1461-1471.

Dai, H., Kaneko, K., Kato, H., Fujii, S., Jing, Y., Xu, A., Sakurai, E., Kato, M., Okamura, N., Kuramasu, A., Yanai, K., 2007. Selective cognitive dysfunction in mice lacking histamine $\mathrm{H} 1$ and $\mathrm{H} 2$ receptors. Neurosci Res 57, 306-313.

Dale HH, L. P., 1910. The physiological action of beta-iminazolylethylamine. J

Physiol. 1910 Dec 31;41(5):318-44. PubMed PMID: 16993030; PubMed Central PMCID: PMC1512903. .

Dantzer, R., 1991. Stress, stereotypies and welfare. Behav Processes 25, 95-102.

Das, A., Rai, D., Dikshit, M., Palit, G., Nath, C., 2005. Nature of stress: differential effects on brain acetylcholinesterase activity and memory in rats. Life Sci 77, 2299-2311.

De Almeida, RMM, Miczek, KA, 2002. Aggression escalated by social instigation or by discontinuation of reinforcement ("frustration") in mice: inhibition by anpirtoline - a 5-HT1 B receptor agonist. . Neuropsychopharmacology.

de Almeida, M. A., Izquierdo, I., 1986. Memory facilitation by histamine. Arch Int Pharmacodyn Ther 283, 193-198.

de Kloet, E. R., Joëls, M., Holsboer, F., 2005. Stress and the brain: from adaptation to disease. Nat Rev Neurosci 6, 463-475.

de Kloet, E. R., Joëls, M., Oitzl, M., Sutanto, W., 1991. Implication of brain corticosteroid receptor diversity for the adaptation syndrome concept. Methods Achiev Exp Pathol 14, 104-132.

De Kloet, E. R., Korte, S. M., Rots, N. Y., Kruk, M. R., 1996. Stress hormones, genotype, and brain organization. Implications for aggression. Ann N Y Acad Sci 794, 179-191.

de Kloet, E. R., Oitzl, M. S., Joëls, M., 1999. Stress and cognition: are corticosteroids good or bad guys? Trends Neurosci 22, 422-426.

de Souza, M. A., Centenaro, L. A., Menegotto, P. R., Henriques, T. P., Bonini, J., Achaval, M., Lucion, A. B., 2013. Prenatal stress produces social behavior deficits and alters 
the number of oxytocin and vasopressin neurons in adult rats. Neurochem Res 38, 1479-1489.

Decker, H. S., 2007. How Kraepelinian was Kraepelin? How Kraepelinian are the neoKraepelinians?--from Emil Kraepelin to DSM-III. Hist Psychiatry 18, 337-360.

Dere, E., Kart-Teke, E., Huston, J. P., De Souza Silva, M. A., 2006. The case for episodic memory in animals. Neurosci Biobehav Rev 30, 1206-1224.

Devito, L. M., Eichenbaum, H., 2011. Memory for the order of events in specific sequences: contributions of the hippocampus and medial prefrontal cortex. J Neurosci 31, 3169-3175.

Dixon, M., King, S., Steiger, H., 1998. The contribution of depression and denial towards understanding the unawareness of symptoms in schizophrenic out-patients. Br J Med Psychol 71 ( Pt 1), 85-97.

Dlugos, A., Childs, E., Stuhr, K. L., Hillard, C. J., de Wit, H., 2012. Acute stress increases circulating anandamide and other $\mathrm{N}$-acylethanolamines in healthy humans. Neuropsychopharmacology 37, 2416-2427.

Doreulee, N., Yanovsky, Y., Flagmeyer, I., Stevens, D. R., Haas, H. L., Brown, R. E., 2001. Histamine $\mathrm{H}(3)$ receptors depress synaptic transmission in the corticostriatal pathway. Neuropharmacology 40, 106-113.

Downing, J. E., Miyan, J. A., 2000. Neural immunoregulation: emerging roles for nerves in immune homeostasis and disease. Immunol Today 21, 281-289.

Drugan, R. C., Skolnick, P., Paul, S. M., Crawley, J. N., 1989. A pretest procedure reliably predicts performance in two animal models of inescapable stress. Pharmacol Biochem Behav 33, 649-654.

Duerkop, B. A., Vaishnava, S., Hooper, L. V., 2009. Immune responses to the microbiota at the intestinal mucosal surface. Immunity 31, 368-376.

Dulawa, S. C., Hen, R., 2005. Recent advances in animal models of chronic antidepressant effects: the novelty-induced hypophagia test. Neurosci Biobehav Rev 29, 771-783.

Duman, R. S., 2004. Depression: a case of neuronal life and death? Biol Psychiatry 56, 140-145.

Duman, R. S., 2005. Neurotrophic factors and regulation of mood: role of exercise, diet and metabolism. Neurobiol Aging 26 Suppl 1, 88-93.

Duman, R. S., Aghajanian, G. K., Sanacora, G., Krystal, J. H., 2016. Synaptic plasticity and depression: new insights from stress and rapid-acting antidepressants. Nat Med 22, 238-249.

Duman, R. S., Nakagawa, S., Malberg, J., 2001. Regulation of adult neurogenesis by antidepressant treatment. Neuropsychopharmacology 25, 836-844.

Eichenbaum, H., 1997. Declarative memory: insights from cognitive neurobiology. Annu Rev Psychol 48, 547-572.

Eichenbaum, H., Yonelinas, A. P., Ranganath, C., 2007. The medial temporal lobe and recognition memory. Annu Rev Neurosci 30, 123-152.

Ennaceur, A., Delacour, J., 1988. A new one-trial test for neurobiological studies of memory in rats. 1: Behavioral data. Behav Brain Res 31, 47-59.

Ercan-Sencicek, A. G., Stillman, A. A., Ghosh, A. K., Bilguvar, K., O’Roak, B. J., Mason, C. E., Abbott, T., Gupta, A., King, R. A., Pauls, D. L., Tischfield, J. A., Heiman, G. A., 
Histaminergic neurotransmission as a gateway for the effects of Oleoylethanolamide

Singer, H. S., Gilbert, D. L., Hoekstra, P. J., Morgan, T. M., Loring, E., Yasuno, K., Fernandez, T., Sanders, S., Louvi, A., Cho, J. H., Mane, S., Colangelo, C. M., Biederer, T., Lifton, R. P., Gunel, M., State, M. W., 2010. L-histidine decarboxylase and Tourette's syndrome. N Engl J Med 362, 1901-1908.

Ericson, H., Blomqvist, A., Köhler, C., 1991. Origin of neuronal inputs to the region of the tuberomammillary nucleus of the rat brain. J Comp Neurol 311, 45-64.

Ericson, H., Watanabe, T., Köhler, C., 1987. Morphological analysis of the tuberomammillary nucleus in the rat brain: delineation of subgroups with antibody against L-histidine decarboxylase as a marker. J Comp Neurol 263, 1-24.

Eriksson, K. S., Sergeeva, O., Brown, R. E., Haas, H. L., 2001. Orexin/hypocretin excites the histaminergic neurons of the tuberomammillary nucleus. J Neurosci 21, 9273-9279.

Eriksson, K. S., Sergeeva, O. A., Selbach, O., Haas, H. L., 2004. Orexin (hypocretin)/ dynorphin neurons control GABAergic inputs to tuberomammillary neurons. Eur J Neurosci 19, 1278-1284.

Esposito, B., De Santis, A., Monteforte, R., Baccari, G. C., 2002. Mast cells in Wallerian degeneration: morphologic and ultrastructural changes. J Comp Neurol 445, 199-210.

Fariñas, I., Jones, K. R., Tessarollo, L., Vigers, A. J., Huang, E., Kirstein, M., de Caprona, D. C., Coppola, V., Backus, C., Reichardt, L. F., Fritzsch, B., 2001. Spatial shaping of cochlear innervation by temporally regulated neurotrophin expression. J Neurosci 21, 6170-6180.

Feder, A., Nestler, E. J., Charney, D. S., 2009. Psychobiology and molecular genetics of resilience. Nat Rev Neurosci 10, 446-457.

Fink, G., 2009. Stress: Definition and history. Elsevier.

Fiorenza, N. G., Rosa, J., Izquierdo, I., Myskiw, J. C., 2012. Modulation of the extinction of two different fear-motivated tasks in three distinct brain areas. Behav Brain Res 232, 210-216.

Fogel, W. A., Tuomisto, L., Sasiak, K., Rokicki, W., Rokicki, M., MacDonald, E., Maslinski, C., 1994. Effect of pargyline on brain N-tele-methylhistamine in portocaval-shunted rats: relation to amine neurotransmitters. J Neurochem 62, 615-620.

Forsythe, P., Bienenstock, J., 2010. Immunomodulation by commensal and probiotic bacteria. Immunol Invest 39, 429-448.

Frade, J. M., Bovolenta, P., Martínez-Morales, J. R., Arribas, A., Barbas, J. A., RodríguezTébar, A., 1997. Control of early cell death by BDNF in the chick retina. Development 124, 3313-3320.

Frankenhaeuser, M., Lundberg, U., Rauste von Wright, M., von Wright, J., Sedvall, G., 1986. Urinary monoamine metabolites as indices of mental stress in healthy males and females. Pharmacol Biochem Behav 24, 1521-1525.

Franklin, T. B., Linder, N., Russig, H., Thöny, B., Mansuy, I. M., 2011. Influence of early stress on social abilities and serotonergic functions across generations in mice. PLoS One 6, e21842.

Fritzsch, B., 2003. Molecular developmental neurobiology of formation, guidance and survival of primary vestibular neurons. Adv Space Res 32, 1495-1500.

Fu, J., Astarita, G., Gaetani, S., Kim, J., Cravatt, B. F., Mackie, K., Piomelli, D., 2007. Food intake regulates oleoylethanolamide formation and degradation in the proximal small intestine. J Biol Chem 282, 1518-1528. 
Fu, J., Dipatrizio, N. V., Guijarro, A., Schwartz, G. J., Li, X., Gaetani, S., Astarita, G., Piomelli, D., 2011. Sympathetic activity controls fat-induced oleoylethanolamide signaling in small intestine. J Neurosci 31, 5730-5736.

Fu, J., Oveisi, F., Gaetani, S., Lin, E., Piomelli, D., 2005. Oleoylethanolamide, an endogenous PPAR-alpha agonist, lowers body weight and hyperlipidemia in obese rats. Neuropharmacology 48, 1147-1153.

Fujise, T., Yoshimatsu, H., Kurokawa, M., Oohara, A., Kang, M., Nakata, M., Sakata, T., 1998. Satiation and masticatory function modulated by brain histamine in rats. Proc Soc Exp Biol Med 217, 228-234.

Fukagawa, K., Sakata, T., Shiraishi, T., Yoshimatsu, H., Fujimoto, K., Ookuma, K., Wada, H., 1989. Neuronal histamine modulates feeding behavior through H1-receptor in rat hypothalamus. Am J Physiol 256, R605-611.

Gabrieli, J. D., 1998. Cognitive neuroscience of human memory. Annu Rev Psychol 49, 87-115.

Gadek-Michalska, A. B., J, 2003. Repeated handling, restraint, or chronic

crowding impair the hypothalamic-pituitary-adrenocortical response to acute

restraint stress. J Physiol Pharmacol., pp. 449-459.

Gaetani, S., Fu, J., Cassano, T., Dipasquale, P., Romano, A., Righetti, L., Cianci, S., Laconca, L., Giannini, E., Scaccianoce, S., Mairesse, J., Cuomo, V., Piomelli, D., 2010. The fatinduced satiety factor oleoylethanolamide suppresses feeding through central release of oxytocin. J Neurosci 30, 8096-8101.

Gaetani, S., Oveisi, F., Piomelli, D., 2003. Modulation of meal pattern in the rat by the anorexic lipid mediator oleoylethanolamide. Neuropsychopharmacology 28, 1311-1316.

Gagne, M. A., Wollin, A., Navert, H., Pinard, G., 1982. Anomaly of histamine methylation in endogenous depression. Prog Neuropsychopharmacol Biol Psychiatry 6, 483-486.

Gamallo, A., Villanua, A., Trancho, G., Fraile, A., 1986. Stress adaptation and adrenal activity in isolated and crowded rats. Physiol Behav 36, 217-221.

Gantner, F., Sakai, K., Tusche, M. W., Cruikshank, W. W., Center, D. M., Bacon, K. B., 2002. Histamine $\mathrm{h}(4)$ and $\mathrm{h}(2)$ receptors control histamine-induced interleukin-16 release from human CD8(+) T cells. J Pharmacol Exp Ther 303, 300-307.

Garbarg, M., Barbin, G., Feger, J., Schwartz, J. C., 1974. Histaminergic pathway in rat brain evidenced by lesions of the medial forebrain bundle. Science 186, 833-835.

Garbarg, M., Javoy-Agid, F., Schwartz, J. C., Agid, Y., 1983. Brain histidine decarboxylase activity in Parkinson's disease. Lancet 1, 74-75.

Gaynes, B. N., Dusetzina, S. B., Ellis, A. R., Hansen, R. A., Farley, J. F., Miller, W. C., Stürmer, T., 2012. Treating depression after initial treatment failure: directly comparing switch and augmenting strategies in STAR ${ }^{\star}$ D. J Clin Psychopharmacol 32, 114-119.

Gerald, M. C., Skau, K. A., Maickel, R. P., 1972. Effects of various antihistamines on oxotremorine-induced hypothermia in mice. Eur J Pharmacol 17, 189-193.

Giannoni, P., Passani, M. B., Nosi, D., Chazot, P. L., Shenton, F. C., Medhurst, A. D., Munari, L., Blandina, P., 2009. Heterogeneity of histaminergic neurons in the tuberomammillary nucleus of the rat. Eur J Neurosci 29, 2363-2374.

Glaser, R., Kiecolt-Glaser, J. K., 2005. Stress-induced immune dysfunction: implications for health. Nat Rev Immunol 5, 243-251. 
Histaminergic neurotransmission as a gateway for the effects of Oleoylethanolamide

Gluckman, P. D., Hanson, M. A., Beedle, A. S., 2007. Early life events and their consequences for later disease: a life history and evolutionary perspective. Am J Hum Biol 19, 1-19.

Gold, P. E., 1986. The use of avoidance training in studies of modulation of memory storage. Behav Neural Biol 46, 87-98.

González-Aparicio, R., Moratalla, R., 2014. Oleoylethanolamide reduces L-DOPA-induced dyskinesia via TRPV1 receptor in a mouse model of Parkinson's disease. Neurobiol Dis 62, 416-425.

Gotoh, K., Fukagawa, K., Fukagawa, T., Noguchi, H., Kakuma, T., Sakata, T., Yoshimatsu, H., 2005. Glucagon-like peptide-1, corticotropin-releasing hormone, and hypothalamic neuronal histamine interact in the leptin-signaling pathway to regulate feeding behavior. FASEB J 19, 1131-1133.

Gould, E., Tanapat, P., 1999. Stress and hippocampal neurogenesis. Biol Psychiatry 46, 1472-1479.

Gould, E., Tanapat, P., McEwen, B. S., Flügge, G., Fuchs, E., 1998. Proliferation of granule cell precursors in the dentate gyrus of adult monkeys is diminished by stress. Proc Natl Acad Sci U S A 95, 3168-3171.

Gray, T. S., Bingaman, E. W., 1996. The amygdala: corticotropin-releasing factor, steroids, and stress. Crit Rev Neurobiol 10, 155-168.

GREEN, J. D., MACHNE, X., 1955. Unit activity of rabbit hippocampus. Am J Physiol 181, 219-224.

Grippo, A. J., Carter, C. S., McNeal, N., Chandler, D. L., Larocca, M. A., Bates, S. L., Porges, S. W., 2011. 24-hour autonomic dysfunction and depressive behaviors in an animal model of social isolation: implications for the study of depression and cardiovascular disease. Psychosom Med 73, 59-66.

Gross, C. T., Canteras, N. S., 2012. The many paths to fear. Nat Rev Neurosci 13, 651-658.

Gunnar, M., Quevedo, K., 2007. The neurobiology of stress and development. Annu Rev Psychol 58, 145-173.

Gutowski, S., Smrcka, A., Nowak, L., Wu, D. G., Simon, M., Sternweis, P. C., 1991. Antibodies to the alpha q subfamily of guanine nucleotide-binding regulatory protein alpha subunits attenuate activation of phosphatidylinositol 4,5-bisphosphate hydrolysis by hormones. J Biol Chem 266, 20519-20524.

Guzowski, J. F., McGaugh, J. L., 1997. Antisense oligodeoxynucleotide-mediated disruption of hippocampal cAMP response element binding protein levels impairs consolidation of memory for water maze training. Proc Natl Acad Sci U S A 94, 2693-2698.

Haas, H., Panula, P., 2003. The role of histamine and the tuberomamillary nucleus in the nervous system. Nat Rev Neurosci 4, 121-130.

Haas, H. L., Sergeeva, O. A., 2012. Warm and awake with histamine. Neuropharmacology 63, 169-170.

Haas, H. L., Sergeeva, O. A., Selbach, O., 2008. Histamine in the nervous system. Physiol Rev 88, 1183-1241.

Haenisch, B., Bilkei-Gorzo, A., Caron, M. G., Bönisch, H., 2009. Knockout of the norepinephrine transporter and pharmacologically diverse antidepressants prevent behavioral and brain neurotrophin alterations in two chronic stress models of depression. J Neurochem 111, 403-416. 
Haller, J., Fuchs, E., Halász, J., Makara, G. B., 1999. Defeat is a major stressor in males while social instability is stressful mainly in females: towards the development of a social stress model in female rats. Brain Res Bull 50, 33-39.

Hancock, A. A., Brune, M. E., 2005. Assessment of pharmacology and potential anti-obesity properties of $\mathrm{H} 3$ receptor antagonists/inverse agonists. Expert Opin Investig Drugs $14,223-241$.

Hannesson, D. K., Vacca, G., Howland, J. G., Phillips, A. G., 2004. Medial prefrontal cortex is involved in spatial temporal order memory but not spatial recognition memory in tests relying on spontaneous exploration in rats. Behav Brain Res 153, 273-285.

Hansen, H. S., Rosenkilde, M. M., Holst, J. J., Schwartz, T. W., 2012. GPR119 as a fat sensor. Trends Pharmacol Sci 33, 374-381.

Hatch, A. M., Wiberg, G. S., Zawidzka, Z., Cann, M., Airth, J. M., Grice, H. C., 1965. Isolation syndrome in the rat. Toxicol Appl Pharmacol 7, 737-745.

Hauer, D., Schelling, G., Gola, H., Campolongo, P., Morath, J., Roozendaal, B., Hamuni, G., Karabatsiakis, A., Atsak, P., Vogeser, M., Kolassa, I. T., 2013. Plasma concentrations of endocannabinoids and related primary fatty acid amides in patients with post-traumatic stress disorder. PLoS One 8, e62741.

Herman, J. P., Cullinan, W. E., Morano, M. I., Akil, H., Watson, S. J., 1995. Contribution of the ventral subiculum to inhibitory regulation of the hypothalamo-pituitary-adrenocortical axis. J Neuroendocrinol 7, 475-482.

Herry, C., Johansen, J. P., 2014. Encoding of fear learning and memory in distributed neuronal circuits. Nat Neurosci 17, 1644-1654.

Herzog, C. J., Czéh, B., Corbach, S., Wuttke, W., Schulte-Herbrüggen, O., Hellweg, R., Flügge, G., Fuchs, E., 2009. Chronic social instability stress in female rats: a potential animal model for female depression. Neuroscience 159, 982-992.

Hill, S. J., 1990. Distribution, properties, and functional characteristics of three classes of histamine receptor. Pharmacol Rev 42, 45-83.

Hill, S. J., Ganellin, C. R., Timmerman, H., Schwartz, J. C., Shankley, N. P., Young, J. M., Schunack, W., Levi, R., Haas, H. L., 1997. International Union of Pharmacology. XIII. Classification of histamine receptors. Pharmacol Rev 49, 253-278.

Hirai, T., Okuma, C., Harada, C., Mio, M., Ohtsu, H., Watanabe, T., Kamei, C., 2004. Development of amygdaloid kindling in histidine decarboxylase-deficient and histamine H1 receptor-deficient mice. Epilepsia 45, 309-313.

Hofstra, C. L., Desai, P. J., Thurmond, R. L., Fung-Leung, W. P., 2003. Histamine H4 receptor mediates chemotaxis and calcium mobilization of mast cells. J Pharmacol Exp Ther 305, 1212-1221.

Hollis, F., Wang, H., Dietz, D., Gunjan, A., Kabbaj, M., 2010. The effects of repeated social defeat on long-term depressive-like behavior and short-term histone modifications in the hippocampus in male Sprague-Dawley rats. Psychopharmacology (Berl) 211, 69-77.

Holtmaat, A., Svoboda, K., 2009. Experience-dependent structural synaptic plasticity in the mammalian brain. Nat Rev Neurosci 10, 647-658.

Hough, L. B., 1988. Cellular localization and possible functions for brain histamine: recent progress. Prog Neurobiol 30, 469-505. 
Histaminergic neurotransmission as a gateway for the effects of Oleoylethanolamide

$\mathrm{Hu}, \mathrm{W}$., Chen, Z., 2017. The roles of histamine and its receptor ligands in central nervous system disorders: An update. Pharmacol Ther 175, 116-132.

Huang, E. J., Reichardt, L. F., 2003. Trk receptors: roles in neuronal signal transduction. Annu Rev Biochem 72, 609-642.

Huang, Y. W., Chen, Z., Hu, W. W., Zhang, L. S., Wu, W., Ying, L. Y., Wei, E. Q., 2003. Facilitating effect of histamine on spatial memory deficits induced by dizocilpine as evaluated by 8-arm radial maze in SD rats. Acta Pharmacol Sin 24, 1270-1276.

Huang, Z. L., Mochizuki, T., Qu, W. M., Hong, Z. Y., Watanabe, T., Urade, Y., Hayaishi, O., 2006. Altered sleep-wake characteristics and lack of arousal response to $\mathrm{H} 3$ receptor antagonist in histamine H1 receptor knockout mice. Proc Natl Acad Sci U S A 103, 4687-4692.

Inagaki, N., Toda, K., Taniuchi, I., Panula, P., Yamatodani, A., Tohyama, M., Watanabe, T., Wada, H., 1990. An analysis of histaminergic efferents of the tuberomammillary nucleus to the medial preoptic area and inferior colliculus of the rat. Exp Brain Res 80, 374-380.

Inagaki, N., Yamatodani, A., Shinoda, K., Panula, P., Watanabe, T., Shiotani, Y., Wada, H., 1988. Histaminergic nerve fibers in the median eminence and hypophysis of rats demonstrated immunocytochemically with antibodies against histidine decarboxylase and histamine. Brain Res 439, 402-405.

Inzunza, O., Serón-Ferré, M. J., Bravo, H., Torrealba, F., 2000. Tuberomammillary nucleus activation anticipates feeding under a restricted schedule in rats. Neurosci Lett 293, 139-142.

Ishizuka, T., Hatano, K., Murotani, T., Yamatodani, A., 2008. Comparison of the effect of an $\mathrm{H}(3)$-inverse agonist on energy intake and hypothalamic histamine release in normal mice and leptin resistant mice with high fat diet-induced obesity. Behav Brain Res $188,250-254$.

Ishizuka, T., Nomura, S., Hosoda, H., Kangawa, K., Watanabe, T., Yamatodani, A., 2006. A role of the histaminergic system for the control of feeding by orexigenic peptides. Physiol Behav 89, 295-300.

Ito, C., 2000. The role of brain histamine in acute and chronic stresses. Biomed Pharmacother 54, 263-267.

Ito, C., 2009. Histamine H3-receptor inverse agonists as novel antipsychotics. Cent Nerv Syst Agents Med Chem 9, 132-136.

Ito, C., Shen, H., Toyota, H., Kubota, Y., Sakurai, E., Watanabe, T., Sato, M., 1999. Effects of the acute and chronic restraint stresses on the central histaminergic neuron system of Fischer rat. Neurosci Lett 262, 143-145.

Izquierdo, I., Bevilaqua, L. R., Rossato, J. I., Bonini, J. S., Da Silva, W. C., Medina, J. H., Cammarota, M., 2006a. The connection between the hippocampal and the striatal memory systems of the brain: a review of recent findings. Neurotox Res 10, 113-121.

Izquierdo, I., Bevilaqua, L. R., Rossato, J. I., Bonini, J. S., Medina, J. H., Cammarota, M., 2006b. Different molecular cascades in different sites of the brain control memory consolidation. Trends Neurosci 29, 496-505.

Izquierdo, I., da Cunha, C., Rosat, R., Jerusalinsky, D., Ferreira, M. B., Medina, J. H., 1992. Neurotransmitter receptors involved in post-training memory processing by the amygdala, medial septum, and hippocampus of the rat. Behav Neural Biol 58, 16-26. 
Izquierdo, I., Furini, C. R., Myskiw, J. C., 2016. Fear Memory. Physiol Rev 96, 695-750.

Izquierdo, I., McGaugh, J. L., 2000. Behavioural pharmacology and its contribution to the molecular basis of memory consolidation. Behav Pharmacol 11, 517-534.

Izquierdo, I., Medina, J. H., 1997. Memory formation: the sequence of biochemical events in the hippocampus and its connection to activity in other brain structures. Neurobiol Learn Mem 68, 285-316.

Izquierdo, I., Medina, J. H., Vianna, M. R., Izquierdo, L. A., Barros, D. M., 1999. Separate mechanisms for short- and long-term memory. Behav Brain Res 103, 1-11.

Iñiguez, S. D., Riggs, L. M., Nieto, S. J., Dayrit, G., Zamora, N. N., Shawhan, K. L., Cruz, B., Warren, B. L., 2014. Social defeat stress induces a depression-like phenotype in adolescent male c57BL/6 mice. Stress 17, 247-255.

Jablonski, S. A., Schreiber, W. B., Westbrook, S. R., Brennan, L. E., Stanton, M. E., 2013. Determinants of novel object and location recognition during development. Behav Brain Res 256, 140-150.

Jacobs, B. L., van Praag, H., Gage, F. H., 2000. Adult brain neurogenesis and psychiatry: a novel theory of depression. Mol Psychiatry 5, 262-269.

Jang, I. S., Rhee, J. S., Watanabe, T., Akaike, N., 2001. Histaminergic modulation of GABAergic transmission in rat ventromedial hypothalamic neurones. J Physiol 534, 791-803.

Jansen, A. S., Nguyen, X. V., Karpitskiy, V., Mettenleiter, T. C., Loewy, A. D., 1995. Central command neurons of the sympathetic nervous system: basis of the fight-or-flight response. Science 270, 644-646.

Jia, R., Tai, F., An, S., Zhang, X., Broders, H., 2009. Effects of neonatal paternal deprivation or early deprivation on anxiety and social behaviors of the adults in mandarin voles. Behavioural Processes 82, 271-278.

Jin, C. Y., Anichtchik, O., Panula, P., 2009. Altered histamine H3 receptor radioligand binding in post-mortem brain samples from subjects with psychiatric diseases. Br J Pharmacol 157, 118-129.

Jin, P., Yu, H. L., Tian-Lan, Zhang, F., Quan, Z. S., 2015. Antidepressant-like effects of oleoylethanolamide in a mouse model of chronic unpredictable mild stress. Pharmacol Biochem Behav 133, 146-154.

Johansen, J. P., Cain, C. K., Ostroff, L. E., LeDoux, J. E., 2011. Molecular mechanisms of fear learning and memory. Cell 147, 509-524.

Jongejan, A., Bruysters, M., Ballesteros, J. A., Haaksma, E., Bakker, R. A., Pardo, L., Leurs, R., 2005. Linking agonist binding to histamine $\mathrm{H} 1$ receptor activation. Nat Chem Biol 1, 98-103.

Josselyn, S. A., Kida, S., Silva, A. J., 2004. Inducible repression of CREB function disrupts amygdala-dependent memory. Neurobiol Learn Mem 82, 159-163.

Jourdi, H., Hsu, Y. T., Zhou, M., Qin, Q., Bi, X., Baudry, M., 2009. Positive AMPA receptor modulation rapidly stimulates BDNF release and increases dendritic mRNA translation. J Neurosci 29, 8688-8697.

Joëls, M., 2006. Corticosteroid effects in the brain: U-shape it. Trends Pharmacol Sci 27, 244-250.

Joëls, M., Pu, Z., Wiegert, O., Oitzl, M. S., Krugers, H. J., 2006. Learning under stress: how does it work? Trends Cogn Sci 10, 152-158. 
Histaminergic neurotransmission as a gateway for the effects of Oleoylethanolamide

Jørgensen, E. A., Knigge, U., Watanabe, T., Warberg, J., Kjaer, A., 2005. Histaminergic neurons are involved in the orexigenic effect of orexin-A. Neuroendocrinology 82, 70-77.

Kafitz, K. W., Rose, C. R., Thoenen, H., Konnerth, A., 1999. Neurotrophin-evoked rapid excitation through TrkB receptors. Nature 401, 918-921.

Kamin, 1957. The retention of an incomplete learned avoidance response . . . J Comp Physiol Psychol pp. 457-460.

Kanamaru, M., Iwase, M., Homma, I., 2001. Neuronal histamine release elicited by hyperthermia mediates tracheal dilation and pressor response. Am J Physiol Regul Integr Comp Physiol 280, R1748-1754.

Kanbayashi, T., Kodama, T., Kondo, H., Satoh, S., Inoue, Y., Chiba, S., Shimizu, T., Nishino, S., 2009. CSF histamine contents in narcolepsy, idiopathic hypersomnia and obstructive sleep apnea syndrome. Sleep 32, 181-187.

Kandel, E. R., Squire, L. R., 2000. Neuroscience: breaking down scientific barriers to the study of brain and mind. Science 290, 1113-1120.

Kano, M., Fukudo, S., Tashiro, A., Utsumi, A., Tamura, D., Itoh, M., Iwata, R., Tashiro, M., Mochizuki, H., Funaki, Y., Kato, M., Hongo, M., Yanai, K., 2004. Decreased histamine $\mathrm{H} 1$ receptor binding in the brain of depressed patients. Eur J Neurosci 20, 803-810.

Katoh, Y., Niimi, M., Yamamoto, Y., Kawamura, T., Morimoto-Ishizuka, T., Sawada, M., Takemori, H., Yamatodani, A., 2001. Histamine production by cultured microglial cells of the mouse. Neurosci Lett 305, 181-184.

Keeney, A., Jessop, D. S., Harbuz, M. S., Marsden, C. A., Hogg, S., Blackburn-Munro, R. E., 2006. Differential effects of acute and chronic social defeat stress on hypothalamicpituitary-adrenal axis function and hippocampal serotonin release in mice. J Neuroendocrinol 18, 330-338.

Kessler, R. C., Berglund, P., Demler, O., Jin, R., Koretz, D., Merikangas, K. R., Rush, A. J., Walters, E. E., Wang, P. S., Replication, N. C. S., 2003. The epidemiology of major depressive disorder: results from the National Comorbidity Survey Replication (NCS-R). JAMA 289, 3095-3105.

Kessler, R. C., Merikangas, K. R., Wang, P. S., 2008. The prevalence and correlates of workplace depression in the national comorbidity survey replication. J Occup Environ Med 50, 381-390.

Kim, J. J., Diamond, D. M., 2002. The stressed hippocampus, synaptic plasticity and lost memories. Nat Rev Neurosci 3, 453-462.

Kim, S. F., Huang, A. S., Snowman, A. M., Teuscher, C., Snyder, S. H., 2007. From the Cover: Antipsychotic drug-induced weight gain mediated by histamine $\mathrm{H} 1$ receptor-linked activation of hypothalamic AMP-kinase. Proc Natl Acad Sci U S A 104, 3456-3459.

Kirschbaum, C., Pirke, K. M., Hellhammer, D. H., 1993. The 'Trier Social Stress Test'--a tool for investigating psychobiological stress responses in a laboratory setting. Neuropsychobiology 28, 76-81.

Kjaer, A., Knigge, U., Bach, F. W., Warberg, J., 1992. Histamine- and stress-induced secretion of ACTH and beta-endorphin: involvement of corticotropin-releasing hormone and vasopressin. Neuroendocrinology 56, 419-428.

Kjaer, A., Knigge, U., Jørgensen, H., Warberg, J., 1998. Dehydration-induced renin secretion: involvement of histaminergic neurons. Neuroendocrinology 67, 325-329. 
Kjaer, A., Knigge, U., Rouleau, A., Garbarg, M., Warberg, J., 1994. Dehydration-induced release of vasopressin involves activation of hypothalamic histaminergic neurons. Endocrinology 135, 675-681.

Kjaer, A., Larsen, P. J., Knigge, U., Warberg, J., 1995. Dehydration stimulates hypothalamic gene expression of histamine synthesis enzyme: importance for neuroendocrine regulation of vasopressin and oxytocin secretion. Endocrinology 136, 2189-2197.

Kolb, B., Buhrmann, K., McDonald, R., Sutherland, R. J., 1994. Dissociation of the medial prefrontal, posterior parietal, and posterior temporal cortex for spatial navigation and recognition memory in the rat. Cereb Cortex 4, 664-680.

Korte, S. M., Koolhaas, J. M., Wingfield, J. C., McEwen, B. S., 2005. The Darwinian concept of stress: benefits of allostasis and costs of allostatic load and the trade-offs in health and disease. Neurosci Biobehav Rev 29, 3-38.

Kotrschal, A., Ilmonen, P., Penn, D. J., 2007. Stress impacts telomere dynamics. Biol Lett 3, 128-130.

Krishnan, V., Nestler, E. J., 2008. The molecular neurobiology of depression. Nature 455, 894-902.

Krishnan, V., Nestler, E. J., 2011. Animal models of depression: molecular perspectives. Curr Top Behav Neurosci 7, 121-147.

Kroeze, W. K., Hufeisen, S. J., Popadak, B. A., Renock, S. M., Steinberg, S., Ernsberger, P., Jayathilake, K., Meltzer, H. Y., Roth, B. L., 2003. H1-histamine receptor affinity predicts short-term weight gain for typical and atypical antipsychotic drugs. Neuropsychopharmacology 28, 519-526.

Krout, K. E., Mettenleiter, T. C., Loewy, A. D., 2003. Single CNS neurons link both central motor and cardiosympathetic systems: a double-virus tracing study. Neuroscience $118,853-866$.

Krüger, P. G., Nyland, H. I., 1995. The role of mast cells and diet in the onset and maintenance of multiple sclerosis: a hypothesis. Med Hypotheses 44, 66-69.

Kuehl, 1957. The identification of N -(2-hydroxyethyl)-palmitamide as a naturally occurring anti-inflammatory agent. J Am Chem Soc. , pp. 5577-5578

Kwiatkowski, H., 1941. Observations on the relation of histamine to reactive hyperaemia. J Physiol 100, 147-158.

Köhler, C. A., da Silva, W. C., Benetti, F., Bonini, J. S., 2011. Histaminergic mechanisms for modulation of memory systems. Neural Plast 2011, 328602.

Lambert, D. M., Vandevoorde, S., Jonsson, K. O., Fowler, C. J., 2002. The palmitoylethanolamide family: a new class of anti-inflammatory agents? Curr Med Chem 9, 663-674.

Lan, H., Vassileva, G., Corona, A., Liu, L., Baker, H., Golovko, A., Abbondanzo, S. J., Hu, W., Yang, S., Ning, Y., Del Vecchio, R. A., Poulet, F., Laverty, M., Gustafson, E. L., Hedrick, J. A., Kowalski, T. J., 2009. GPR119 is required for physiological regulation of glucagon-like peptide- 1 secretion but not for metabolic homeostasis. J Endocrinol 201, 219-230.

Lauffer, L. M., Iakoubov, R., Brubaker, P. L., 2009. GPR119 is essential for oleoylethanolamide-induced glucagon-like peptide-1 secretion from the intestinal enteroendocrine L-cell. Diabetes 58, 1058-1066.

LeDoux, J. E., 2014. Coming to terms with fear. Proc Natl Acad Sci U S A 111, 2871-2878. 
Histaminergic neurotransmission as a gateway for the effects of Oleoylethanolamide

Lee, R., Kermani, P., Teng, K. K., Hempstead, B. L., 2001. Regulation of cell survival by secreted proneurotrophins. Science 294, 1945-1948.

Leger, M., Quiedeville, A., Bouet, V., Haelewyn, B., Boulouard, M., Schumann-Bard, P., Freret, T., 2013. Object recognition test in mice. Nat Protoc 8, 2531-2537.

Leibowitz, S. F., 1973. Histamine: a stimulatory effect on drinking behavior in the rat. Brain Res 63, 440-444.

Leopoldt, D., Harteneck, C., Nürnberg, B., 1997. G proteins endogenously expressed in Sf 9 cells: interactions with mammalian histamine receptors. Naunyn Schmiedebergs Arch Pharmacol 356, 216-224.

Leshner, A. I., Remler, H., Biegon, A., Samuel, D., 1979. Desmethylimipramine (DMI) counteracts learned helplessness in rats. Psychopharmacology (Berl) 66, 207-208.

Leurs, R., Chazot, P. L., Shenton, F. C., Lim, H. D., de Esch, I. J., 2009. Molecular and biochemical pharmacology of the histamine H4 receptor. Br J Pharmacol 157, 14-23.

LEWIS T., G. R. T., 1924. Vascular reactions of the skin to injury. Part 11. The liberation of histamine-like substance in the injured skin, the underlying cause of factitious urticaria and of wheals produced by burning: and observations upon the nervous control of certain skin reactions., Heart. , pp. 209-265.

Li, L., Chen, L., Lin, X., Xu, Y., Ren, J., Fu, J., Qiu, Y., 2015. Effect of oleoylethanolamide on diet-induced nonalcoholic fatty liver in rats. J Pharmacol Sci 127, 244-250.

Lin, J. S., 2000. Brain structures and mechanisms involved in the control of cortical activation and wakefulness, with emphasis on the posterior hypothalamus and histaminergic neurons. Sleep Med Rev 4, 471-503.

Lin, L., Wisor, J., Shiba, T., Taheri, S., Yanai, K., Wurts, S., Lin, X., Vitaterna, M., Takahashi, J., Lovenberg, T. W., Koehl, M., Uhl, G., Nishino, S., Mignot, E., 2002. Measurement of hypocretin/orexin content in the mouse brain using an enzyme immunoassay: the effect of circadian time, age and genetic background. Peptides 23, 2203-2211.

Liu, C., Ma, X., Jiang, X., Wilson, S. J., Hofstra, C. L., Blevitt, J., Pyati, J., Li, X., Chai, W., Carruthers, N., Lovenberg, T. W., 2001. Cloning and pharmacological characterization of a fourth histamine receptor $(\mathrm{H}(4))$ expressed in bone marrow. Mol Pharmacol 59, 420-426.

Liu, R. J., Lee, F. S., Li, X. Y., Bambico, F., Duman, R. S., Aghajanian, G. K., 2012. Brainderived neurotrophic factor Val66Met allele impairs basal and ketamine-stimulated synaptogenesis in prefrontal cortex. Biol Psychiatry 71, 996-1005.

Lo Verme, J., Gaetani, S., Fu, J., Oveisi, F., Burton, K., Piomelli, D., 2005. Regulation of food intake by oleoylethanolamide. Cell Mol Life Sci 62, 708-716.

Lorenzini, C. A., Baldi, E., Bucherelli, C., Sacchetti, B., Tassoni, G., 1996a. Role of dorsal hippocampus in acquisition, consolidation and retrieval of rat's passive avoidance response: a tetrodotoxin functional inactivation study. Brain Res 730, 32-39.

Lorenzini, C. A., Baldi, E., Bucherelli, C., Tassoni, G., 1996b. Amnesic effects of preacquisition, postacquisition, or preretrieval tetrodotoxin administration into the medial septal area on rat's passive avoidance memorization. Neurobiol Learn Mem 66, 80-84.

Lucas, M., Ilin, Y., Anunu, R., Kehat, O., Xu, L., Desmedt, A., Richter-Levin, G., 2014. Long-term effects of controllability or the lack of it on coping abilities and stress resilience in the rat. Stress 17, 423-430. 
Lucki, I., 2001. A prescription to resist proscriptions for murine models of depression. Psychopharmacology (Berl) 153, 395-398.

Luine, V., 2002. Sex differences in chronic stress effects on memory in rats. Stress 5, 205-216.

Machidori, H., Sakata, T., Yoshimatsu, H., Ookuma, K., Fujimoto, K., Kurokawa, M., Yamatodani, A., Wada, H., 1992. Zucker obese rats: defect in brain histamine control of feeding. Brain Res 590, 180-186.

MACHNE, X., SEGUNDO, J. P., 1956. Unitary responses to afferent volleys in amygdaloid complex. J Neurophysiol 19, 232-240.

Magariños, A. M., Li, C. J., Gal Toth, J., Bath, K. G., Jing, D., Lee, F. S., McEwen, B. S., 2011. Effect of brain-derived neurotrophic factor haploinsufficiency on stress-induced remodeling of hippocampal neurons. Hippocampus 21, 253-264.

Magariños, A. M., McEwen, B. S., Flügge, G., Fuchs, E., 1996. Chronic psychosocial stress causes apical dendritic atrophy of hippocampal CA3 pyramidal neurons in subordinate tree shrews. J Neurosci 16, 3534-3540.

Maier, S. F., Watkins, L. R., 2005. Stressor controllability and learned helplessness: the roles of the dorsal raphe nucleus, serotonin, and corticotropin-releasing factor. Neurosci Biobehav Rev 29, 829-841.

Malberg, J. E., Blendy, J. A., 2005. Antidepressant action: to the nucleus and beyond. Trends Pharmacol Sci 26, 631-638.

Malberg, J. E., Eisch, A. J., Nestler, E. J., Duman, R. S., 2000. Chronic antidepressant treatment increases neurogenesis in adult rat hippocampus. J Neurosci 20, 9104-9110.

Mann, J. J., 2005. The medical management of depression. N Engl J Med 353, 1819-1834.

Martin, P., Beninger, R. J., Hamon, M., Puech, A. J., 1990a. Antidepressant-like action of 8-OH-DPAT, a 5-HT1A agonist, in the learned helplessness paradigm: evidence for a postsynaptic mechanism. Behav Brain Res 38, 135-144.

Martin, P., Soubrié, P., Puech, A. J., 1990b. Reversal of helpless behavior by serotonin uptake blockers in rats. Psychopharmacology (Berl) 101, 403-407.

Martinez, M., Phillips, P. J., Herbert, J., 1998. Adaptation in patterns of c-fos expression in the brain associated with exposure to either single or repeated social stress in male rats. Eur J Neurosci 10, 20-33.

Masaki, T., Yoshimatsu, H., 2006. The hypothalamic H1 receptor: a novel therapeutic target for disrupting diurnal feeding rhythm and obesity. Trends Pharmacol Sci 27, 279-284.

Matsumoto, T., Rauskolb, S., Polack, M., Klose, J., Kolbeck, R., Korte, M., Barde, Y. A., 2008. Biosynthesis and processing of endogenous BDNF: CNS neurons store and secrete BDNF, not pro-BDNF. Nat Neurosci 11, 131-133.

Matzen, S., Knigge, U., Warberg, J., 1990. Brain regulation of renin secretion involves central histaminergic neurons. Neuroendocrinology 52, 175-180.

Mawdsley, J. E., Rampton, D. S., 2006. The role of psychological stress in inflammatory bowel disease. Neuroimmunomodulation 13, 327-336.

McDonald, R. J., Hong, N. S., 2013. How does a specific learning and memory system in the mammalian brain gain control of behavior? Hippocampus 23, 1084-1102.

McEwen, B. S., 1999. Stress and hippocampal plasticity. Annu Rev Neurosci 22, 105-122.

McEwen, B. S., 2000. The neurobiology of stress: from serendipity to clinical relevance. Brain Res 886, 172-189. 
Histaminergic neurotransmission as a gateway for the effects of Oleoylethanolamide

McEwen, B. S., 2003. Mood disorders and allostatic load. Biol Psychiatry 54, 200-207.

McEwen, B. S., 2012. Brain on stress: how the social environment gets under the skin. Proc Natl Acad Sci U S A 109 Suppl 2, 17180-17185.

McEwen, B. S., 2016. Stress-induced remodeling of hippocampal CA3 pyramidal neurons. Brain Res 1645, 50-54.

McEwen, B. S., Magarinos, A. M., 2001. Stress and hippocampal plasticity: implications for the pathophysiology of affective disorders. Hum Psychopharmacol 16, S7-S19.

McEwen, B. S., Sapolsky, R. M., 1995. Stress and cognitive function. Curr Opin Neurobiol $5,205-216$.

McEwen, B. S., Seeman, T., 1999. Protective and damaging effects of mediators of stress. Elaborating and testing the concepts of allostasis and allostatic load. Ann N Y Acad Sci 896, 30-47.

McEwen, B. S., Wingfield, J. C., 2003. The concept of allostasis in biology and biomedicine. Horm Behav 43, 2-15.

McGaugh, J. L., 2000. Memory--a century of consolidation. Science 287, 248-251.

McGaugh, J. L., 2004. The amygdala modulates the consolidation of memories of emotionally arousing experiences. Annu Rev Neurosci 27, 1-28.

McGaugh, J. L., 2015. Consolidating memories. Annu Rev Psychol 66, 1-24.

McGaugh, J. L., Roozendaal, B., 2002. Role of adrenal stress hormones in forming lasting memories in the brain. Curr Opin Neurobiol 12, 205-210.

Merighi, A., Salio, C., Ghirri, A., Lossi, L., Ferrini, F., Betelli, C., Bardoni, R., 2008. BDNF as a pain modulator. Prog Neurobiol 85, 297-317.

Meyer, A., 1922. Interrelations of the domain of neuropsychiatry. Arch Neurol Psychiatry, pp. 111-121.

Miczek, K. A., de Boer, S. F., Haller, J., 2013. Excessive aggression as model of violence: a critical evaluation of current preclinical methods. Psychopharmacology (Berl) 226, 445-458.

Miklós, I. H., Kovács, K. J., 2003. Functional heterogeneity of the responses of histaminergic neuron subpopulations to various stress challenges. Eur J Neurosci 18, 3069-3079.

Mineka, S., RW, 1985. Controllability and predictability in acquired motivation.. . Annu Rev Psychol pp. 495-529.

Mineur, Y. S., Prasol, D. J., Belzung, C., Crusio, W. E., 2003. Agonistic behavior and unpredictable chronic mild stress in mice. Behav Genet 33, 513-519.

Mirescu, C., Gould, E., 2006. Stress and adult neurogenesis. Hippocampus 16, 233-238.

Misra, S., 2012. Cyclic AMP response element binding protein (CREB) in depression: A

new role of an old molecule. Current Neurobiology, pp. 3-6.

Mitchell, J., Mayeenuddin, L. H., 1998. Purification, G protein activation, and partial amino acid sequence of a novel phospholipase $\mathrm{C}$ from squid photoreceptors. Biochemistry 37, 9064-9072.

Mitchell, J. B., Laiacona, J., 1998. The medial frontal cortex and temporal memory: tests using spontaneous exploratory behaviour in the rat. Behav Brain Res 97, 107-113. 
Mobarakeh, J. I., Takahashi, K., Sakurada, S., Kuramasu, A., Yanai, K., 2006. Enhanced antinociceptive effects of morphine in histamine $\mathrm{H} 2$ receptor gene knockout mice. Neuropharmacology 51, 612-622.

Mobarakeh, J. I., Takahashi, K., Sakurada, S., Nishino, S., Watanabe, H., Kato, M., Naghdi, N., Yanai, K., 2005. Enhanced antinociception by intracerebroventricularly administered orexin A in histamine $\mathrm{H} 1$ or $\mathrm{H} 2$ receptor gene knockout mice. Pain 118, 254-262.

Moniri, N. H., Covington-Strachan, D., Booth, R. G., 2004. Ligand-directed functional heterogeneity of histamine $\mathrm{H} 1$ receptors: novel dual-function ligands selectively activate and block H1-mediated phospholipase $\mathrm{C}$ and adenylyl cyclase signaling. J Pharmacol Exp Ther 311, 274-281.

Montiel-Castro, A. J., González-Cervantes, R. M., Bravo-Ruiseco, G., Pacheco-López, G., 2013. The microbiota-gut-brain axis: neurobehavioral correlates, health and sociality. Front Integr Neurosci 7, 70.

Moreau, J. L., Jenck, F., Martin, J. R., Mortas, P., Haefely, W. E., 1992. Antidepressant treatment prevents chronic unpredictable mild stress-induced anhedonia as assessed by ventral tegmentum self-stimulation behavior in rats. Eur Neuropsychopharmacol 2, 43-49.

Morse, K. L., Behan, J., Laz, T. M., West, R. E., Greenfeder, S. A., Anthes, J. C., Umland, S., Wan, Y., Hipkin, R. W., Gonsiorek, W., Shin, N., Gustafson, E. L., Qiao, X., Wang, S., Hedrick, J. A., Greene, J., Bayne, M., Monsma, F. J., 2001. Cloning and characterization of a novel human histamine receptor. J Pharmacol Exp Ther 296, 1058-1066.

Muir, J. L., 1996. Attention and stimulus processing in the rat. Brain Res Cogn Brain Res $3,215-225$.

Munari, L., Provensi, G., Passani, M. B., Galeotti, N., Cassano, T., Benetti, F., Corradetti, R., Blandina, P., 2015. Brain Histamine Is Crucial for Selective Serotonin Reuptake Inhibitors' Behavioral and Neurochemical Effects. Int J Neuropsychopharmacol 18, pyv045.

Murray, L. J., Ranganath, C., 2007. The dorsolateral prefrontal cortex contributes to successful relational memory encoding. J Neurosci 27, 5515-5522.

Musazzi, L., Tornese, P., Sala, N., Popoli, M., 2017. Acute or Chronic? A Stressful Question. Trends Neurosci 40, 525-535.

Muscat, R., Papp, M., Willner, P., 1992. Antidepressant-like effects of dopamine agonists in an animal model of depression. Biol Psychiatry 31, 937-946.

Muscat, R., Sampson, D., Willner, P., 1990. Dopaminergic mechanism of imipramine action in an animal model of depression. Biol Psychiatry 28, 223-230.

Márquez, C., Poirier, G. L., Cordero, M. I., Larsen, M. H., Groner, A., Marquis, J., Magistretti, P. J., Trono, D., Sandi, C., 2013. Peripuberty stress leads to abnormal aggression, altered amygdala and orbitofrontal reactivity and increased prefrontal MAOA gene expression. Transl Psychiatry 3, e216.

Naert, A., Callaerts-Vegh, Z., D’Hooge, R., 2011. Nocturnal hyperactivity, increased social novelty preference and delayed extinction of fear responses in post-weaning socially isolated mice. Brain Res Bull 85, 354-362.

Nair, A., Vaidya, V. A., 2006. Cyclic AMP response element binding protein and brain-derived neurotrophic factor: molecules that modulate our mood? J Biosci 31, 423-434. 
Histaminergic neurotransmission as a gateway for the effects of Oleoylethanolamide

Nakai, T., Kitamura, N., Hashimoto, T., Kajimoto, Y., Nishino, N., Mita, T., Tanaka, C., 1991. Decreased histamine H1 receptors in the frontal cortex of brains from patients with chronic schizophrenia. Biol Psychiatry 30, 349-356.

Nakamura, S., Ohnishi, K., Nishimura, M., Suenaga, T., Akiguchi, I., Kimura, J., Kimura, T., 1996. Large neurons in the tuberomammillary nucleus in patients with Parkinson's disease and multiple system atrophy. Neurology 46, 1693-1696.

Nakamura, T., Itadani, H., Hidaka, Y., Ohta, M., Tanaka, K., 2000. Molecular cloning and characterization of a new human histamine receptor, HH4R. Biochem Biophys Res Commun 279, 615-620.

Nath, C., Gulati, A., Dhawan, K. N., Gupta, G. P., 1988. Role of central histaminergic mechanism in behavioural depression (swimming despair) in mice. Life Sci 42, 2413-2417.

Nelson, L. E., Guo, T. Z., Lu, J., Saper, C. B., Franks, N. P., Maze, M., 2002. The sedative component of anesthesia is mediated by GABA(A) receptors in an endogenous sleep pathway. Nat Neurosci 5, 979-984.

Nelson, L. E., Lu, J., Guo, T., Saper, C. B., Franks, N. P., Maze, M., 2003. The alpha2-adrenoceptor agonist dexmedetomidine converges on an endogenous sleep-promoting pathway to exert its sedative effects. Anesthesiology 98, 428-436.

Nemeroff, C. B., Bremner, J. D., Foa, E. B., Mayberg, H. S., North, C. S., Stein, M. B., 2006. Posttraumatic stress disorder: a state-of-the-science review. J Psychiatr Res 40, 1-21.

Nephew, B. C., Bridges, R. S., 2011. Effects of chronic social stress during lactation on maternal behavior and growth in rats. Stress 14, 677-684.

Nestler, E. J., Barrot, M., DiLeone, R. J., Eisch, A. J., Gold, S. J., Monteggia, L. M., 2002. Neurobiology of depression. Neuron 34, 13-25.

Neto, F. L., Borges, G., Torres-Sanchez, S., Mico, J. A., Berrocoso, E., 2011. Neurotrophins role in depression neurobiology: a review of basic and clinical evidence. Curr Neuropharmacol 9, 530-552.

Netto, C. A., Izquierdo, I., 1985. Posterior hypothalamic deafferentation abolishes the amnestic effect of electroconvulsive shock in rats. Psychoneuroendocrinology 10, 159-163.

Nguyen, T., Shapiro, D. A., George, S. R., Setola, V., Lee, D. K., Cheng, R., Rauser, L., Lee, S. P., Lynch, K. R., Roth, B. L., O’Dowd, B. F., 2001. Discovery of a novel member of the histamine receptor family. Mol Pharmacol 59, 427-433.

Nibuya, M., Morinobu, S., Duman, R. S., 1995. Regulation of BDNF and trkB mRNA in rat brain by chronic electroconvulsive seizure and antidepressant drug treatments. J Neurosci $15,7539-7547$.

Nibuya, M., Nestler, E. J., Duman, R. S., 1996. Chronic antidepressant administration increases the expression of cAMP response element binding protein (CREB) in rat hippocampus. J Neurosci 16, 2365-2372.

Nieuwenhuys, R., Voogd, J., van Huijzen, C., 2008. Diencephalon: Hypothalamus. In: The Human Central Nervous System. Springer, Berlin, Heidelberg.

Nishino, S., Sakurai, E., Nevsimalova, S., Yoshida, Y., Watanabe, T., Yanai, K., Mignot, E., 2009. Decreased CSF histamine in narcolepsy with and without low CSF hypocretin-1 in comparison to healthy controls. Sleep 32, 175-180.

Nowak, P., Bortel, A., Dabrowska, J., Biedka, I., Slomian, G., Roczniak, W., Kostrzewa, R. M., Brus, R., 2008. Histamine H(3) receptor ligands modulate L-dopa-evoked beha- 
vioral responses and L-dopa derived extracellular dopamine in dopamine-denervated rat striatum. Neurotox Res 13, 231-240.

O'Keefe, J., L, 1978 The hippocampus as a cognitive map. . Oxford University Press; Oxford, UK.

O’Neil, E. B., Protzner, A. B., McCormick, C., McLean, D. A., Poppenk, J., Cate, A. D., Köhler, S., 2012. Distinct patterns of functional and effective connectivity between perirhinal cortex and other cortical regions in recognition memory and perceptual discrimination. Cereb Cortex 22, 74-85.

O’Reilly, M., Alpert, R., Jenkinson, S., Gladue, R. P., Foo, S., Trim, S., Peter, B., Trevethick, M., Fidock, M., 2002. Identification of a histamine H4 receptor on human eosinophils-role in eosinophil chemotaxis. J Recept Signal Transduct Res 22, 431-448.

Oda, T., Morikawa, N., Saito, Y., Masuho, Y., Matsumoto, S., 2000. Molecular cloning and characterization of a novel type of histamine receptor preferentially expressed in leukocytes. J Biol Chem 275, 36781-36786.

Okamoto, Y., Wang, J., Morishita, J., Ueda, N., 2007. Biosynthetic pathways of the endocannabinoid anandamide. Chem Biodivers 4, 1842-1857.

Ookuma, K., Sakata, T., Fukagawa, K., Yoshimatsu, H., Kurokawa, M., Machidori, H., Fujimoto, K., 1993. Neuronal histamine in the hypothalamus suppresses food intake in rats. Brain Res 628, 235-242.

Ookuma, K., Yoshimatsu, H., Sakata, T., Fujimoto, K., Fukagawa, F., 1989. Hypothalamic sites of neuronal histamine action on food intake by rats. Brain Res 490, 268-275.

Overmier, J. B., Seligman, M. E., 1967. Effects of inescapable shock upon subsequent escape and avoidance responding. J Comp Physiol Psychol 63, 28-33.

Packard, M. G., Goodman, J., 2013. Factors that influence the relative use of multiple memory systems. Hippocampus 23, 1044-1052.

Palanza, P., Gioiosa, L., Parmigiani, S., 2001. Social stress in mice: gender differences and effects of estrous cycle and social dominance. Physiol Behav 73, 411-420.

Palkovits, M., 1987. Organization of the stress response at the anatomical level. Prog Brain Res 72, 47-55.

Pandey, S. C., 2003. Anxiety and alcohol abuse disorders: a common role for CREB and its target, the neuropeptide Y gene. Trends Pharmacol Sci 24, 456-460.

Panula, P., Chazot, P. L., Cowart, M., Gutzmer, R., Leurs, R., Liu, W. L., Stark, H., Thurmond, R. L., Haas, H. L., 2015. International Union of Basic and Clinical Pharmacology. XCVIII. Histamine Receptors. Pharmacol Rev 67, 601-655.

Panula, P., Nuutinen, S., 2013. The histaminergic network in the brain: basic organization and role in disease. Nat Rev Neurosci 14, 472-487.

Panula, P., Pirvola, U., Auvinen, S., Airaksinen, M. S., 1989. Histamine-immunoreactive nerve fibers in the rat brain. Neuroscience 28, 585-610.

Panula, P., Rinne, J., Kuokkanen, K., Eriksson, K. S., Sallmen, T., Kalimo, H., Relja, M., 1998. Neuronal histamine deficit in Alzheimer's disease. Neuroscience 82, 993-997.

Panula, P., Yang, H. Y., Costa, E., 1984. Histamine-containing neurons in the rat hypothalamus. Proc Natl Acad Sci U S A 81, 2572-2576.

Papp, M., Moryl, E., Willner, P., 1996. Pharmacological validation of the chronic mild stress model of depression. Eur J Pharmacol 296, 129-136. 
Histaminergic neurotransmission as a gateway for the effects of Oleoylethanolamide

Papp, M., Willner, P., Muscat, R., 1991. An animal model of anhedonia: attenuation of sucrose consumption and place preference conditioning by chronic unpredictable mild stress. Psychopharmacology (Berl) 104, 255-259.

Parihar, V. K., Hattiangady, B., Kuruba, R., Shuai, B., Shetty, A. K., 2011. Predictable chronic mild stress improves mood, hippocampal neurogenesis and memory. Mol Psychiatry 16, 171-183.

Parker, A., Eacott, M. J., Gaffan, D., 1997. The recognition memory deficit caused by mediodorsal thalamic lesion in non-human primates: a comparison with rhinal cortex lesion. Eur J Neurosci 9, 2423-2431.

Parmentier, R., Ohtsu, H., Djebbara-Hannas, Z., Valatx, J. L., Watanabe, T., Lin, J. S., 2002. Anatomical, physiological, and pharmacological characteristics of histidine decarboxylase knock-out mice: evidence for the role of brain histamine in behavioral and sleep-wake control. J Neurosci 22, 7695-7711.

Parsons, R. G., Ressler, K. J., 2013. Implications of memory modulation for post-traumatic stress and fear disorders. Nat Neurosci 16, 146-153.

Passani, M. B., Benetti, F., Blandina, P., Furini, C. R. G., de Carvalho Myskiw, J., Izquierdo, I., 2017. Histamine regulates memory consolidation. Neurobiol Learn Mem 145, 1-6.

Passani, M. B., Blandina, P., 1998. Cognitive implications for H3 and 5-HT3 receptor modulation of cortical cholinergic function: a parallel story. Methods Find Exp Clin Pharmacol 20, 725-733.

Passani, M. B., Blandina, P., 2011. Histamine receptors in the CNS as targets for therapeutic intervention. Trends Pharmacol Sci 32, 242-249.

Passani, M. B., Giannoni, P., Bucherelli, C., Baldi, E., Blandina, P., 2007. Histamine in the brain: beyond sleep and memory. Biochem Pharmacol 73, 1113-1122.

Patton, M. H., Bizup, B. T., Grace, A. A., 2013. The infralimbic cortex bidirectionally modulates mesolimbic dopamine neuron activity via distinct neural pathways. J Neurosci 33, 16865-16873.

Pawar, A., Jump, D. B., 2003. Unsaturated fatty acid regulation of peroxisome proliferator-activated receptor alpha activity in rat primary hepatocytes. J Biol Chem 278, 35931-35939.

Paxinos, G, Watson, C, 1998. The rat brain in stereotaxic coordinates . . New York: Academic Press

Paykel, E. S., 2008. Basic concepts of depression. Dialogues Clin Neurosci 10, 279-289.

Pecoraro, N., Gomez, F., Fleur, S. 1., Roy, M., Dallman, M. F., 2005. Single, but not multiple pairings of sucrose and corticosterone enhance memory for sucrose drinking and amplify remote reward relativity effects. Neurobiology of Learning and Memory 83, 188-195.

Perlis, R. H., Adams, D. H., Fijal, B., Sutton, V. K., Farmen, M., Breier, A., Houston, J. P., 2010. Genetic association study of treatment response with olanzapine/fluoxetine combination or lamotrigine in bipolar I depression. J Clin Psychiatry 71, 599-605.

Peters, S., Grunwald, N., Rümmele, P., Endlicher, E., Lechner, A., Neumann, I. D., Obermeier, F., Reber, S. O., 2012. Chronic psychosocial stress increases the risk for inflammation-related colon carcinogenesis in male mice. Stress 15, 403-415.

Petty, F., Sherman, A. D., 1979. Reversal of learned helplessness by imipramine. Commun Psychopharmacol 3, 371-373. 
Pinnock, S. B., Herbert, J., 2001. Corticosterone differentially modulates expression of corticotropin releasing factor and arginine vasopressin mRNA in the hypothalamic paraventricular nucleus following either acute or repeated restraint stress. Eur J Neurosci $13,576-584$.

Piomelli, D., 2013. A fatty gut feeling. Trends Endocrinol Metab 24, 332-341.

Pizzagalli, D. A., 2014. Depression, stress, and anhedonia: toward a synthesis and integrated model. Annu Rev Clin Psychol 10, 393-423.

Pliakas, A. M., Carlson, R. R., Neve, R. L., Konradi, C., Nestler, E. J., Carlezon, W. A., 2001. Altered responsiveness to cocaine and increased immobility in the forced swim test associated with elevated cAMP response element-binding protein expression in nucleus accumbens. J Neurosci 21, 7397-7403.

Poldrack, R. A., Packard, M. G., 2003. Competition among multiple memory systems: converging evidence from animal and human brain studies. Neuropsychologia 41, 245-251.

Pollak, D. D., Rey, C. E., Monje, F. J., 2010. Rodent models in depression research: classical strategies and new directions. Ann Med 42, 252-264.

Porsolt, R. D., 2000. Animal models of depression: utility for transgenic research. Rev Neurosci 11, 53-58.

Porsolt, R. D., Le Pichon, M., Jalfre, M., 1977. Depression: a new animal model sensitive to antidepressant treatments. Nature 266, 730-732.

Porsolt, R. D., Lenegre, A., 1992. Behavioural models of depression. . Wiley, London, pp. 73-85.

Prell, G. D., Green, J. P., Kaufmann, C. A., Khandelwal, J. K., Morrishow, A. M., Kirch, D. G., Linnoila, M., Wyatt, R. J., 1995. Histamine metabolites in cerebrospinal fluid of patients with chronic schizophrenia: their relationships to levels of other aminergic transmitters and ratings of symptoms. Schizophr Res 14, 93-104.

Prell, G. D., Khandelwal, J. K., Burns, R. S., LeWitt, P. A., Green, J. P., 1988. Elevated levels of histamine metabolites in cerebrospinal fluid of aging, healthy humans. Compr Gerontol A 2, 114-119.

Proulx, K., Cota, D., Castañeda, T. R., Tschöp, M. H., D’Alessio, D. A., Tso, P., Woods, S. C., Seeley, R. J., 2005. Mechanisms of oleoylethanolamide-induced changes in feeding behavior and motor activity. Am J Physiol Regul Integr Comp Physiol 289, R729-737.

Provensi, G., Blandina, P., Passani, M. B., 2016. The histaminergic system as a target for the prevention of obesity and metabolic syndrome. Neuropharmacology 106, 3-12.

Provensi, G., Coccurello, R., Umehara, H., Munari, L., Giacovazzo, G., Galeotti, N., Nosi, D., Gaetani, S., Romano, A., Moles, A., Blandina, P., Passani, M. B., 2014. Satiety factor oleoylethanolamide recruits the brain histaminergic system to inhibit food intake. Proc Natl Acad Sci U S A 111, 11527-11532.

Provensi, G., Fabbri, R., Munari, L., Costa, A., Baldi, E., Bucherelli, C., Blandina, P., Passani, M. B., 2017. Histaminergic Neurotransmission as a Gateway for the Cognitive Effect of Oleoylethanolamide in Contextual Fear Conditioning. Int J Neuropsychopharmacol 20, 392-399.

Puzserova, A., Slezak, P., Balis, P., Bernatova, I., 2013. Long-term social stress induces nitric oxide-independent endothelial dysfunction in normotensive rats. Stress 16, 331-339. 
Histaminergic neurotransmission as a gateway for the effects of Oleoylethanolamide

Quirici, V, Faugeron, S, Hayes, D, L., Ebensperger, A., L., 2011. The influence of group size on natal dispersal in the communally rearing and semifossorial rodent, Octodon degus . Behavioral Ecology and Sociobiology, pp. 787-798.

Radley, J. J., Sisti, H. M., Hao, J., Rocher, A. B., McCall, T., Hof, P. R., McEwen, B. S., Morrison, J. H., 2004. Chronic behavioral stress induces apical dendritic reorganization in pyramidal neurons of the medial prefrontal cortex. Neuroscience 125, 1-6.

Ragozzino, M. E., Detrick, S., Kesner, R. P., 2002. The effects of prelimbic and infralimbic lesions on working memory for visual objects in rats. Neurobiol Learn Mem 77, 29-43.

Renard, C. E., Dailly, E., David, D. J., Hascoet, M., Bourin, M., 2003. Monoamine metabolism changes following the mouse forced swimming test but not the tail suspension test. Fundam Clin Pharmacol 17, 449-455.

Rhee, S. H., Pothoulakis, C., Mayer, E. A., 2009. Principles and clinical implications of the brain-gut-enteric microbiota axis. Nat Rev Gastroenterol Hepatol 6, 306-314.

Rinne, J. O., Anichtchik, O. V., Eriksson, K. S., Kaslin, J., Tuomisto, L., Kalimo, H., Röyttä, M., Panula, P., 2002. Increased brain histamine levels in Parkinson's disease but not in multiple system atrophy. J Neurochem 81, 954-960.

Rodríguez de Fonseca, F., Navarro, M., Gómez, R., Escuredo, L., Nava, F., Fu, J., MurilloRodríguez, E., Giuffrida, A., LoVerme, J., Gaetani, S., Kathuria, S., Gall, C., Piomelli, D., 2001. An anorexic lipid mediator regulated by feeding. Nature 414, 209-212.

Romano, A., Coccurello, R., Giacovazzo, G., Bedse, G., Moles, A., Gaetani, S., 2014. Oleoylethanolamide: a novel potential pharmacological alternative to cannabinoid antagonists for the control of appetite. Biomed Res Int 2014, 203425.

Roozendaal, B., 2002. Stress and memory: opposing effects of glucocorticoids on memory consolidation and memory retrieval. Neurobiol Learn Mem 78, 578-595.

Roozendaal, B., 2003. Systems mediating acute glucocorticoid effects on memory consolidation and retrieval. Prog Neuropsychopharmacol Biol Psychiatry 27, 1213-1223.

Rutter, M., 2006. Implications of resilience concepts for scientific understanding. Ann N Y Acad Sci 1094, 1-12.

Ryu, J. H., Yanai, K., Watanabe, T., 1994. Marked increase in histamine H3 receptors in the striatum and substantia nigra after 6-hydroxydopamine-induced denervation of dopaminergic neurons: an autoradiographic study. Neurosci Lett 178, 19-22.

Rösch, H., Schweigreiter, R., Bonhoeffer, T., Barde, Y. A., Korte, M., 2005. The neurotrophin receptor p75NTR modulates long-term depression and regulates the expression of AMPA receptor subunits in the hippocampus. Proc Natl Acad Sci U S A 102, 7362-7367.

Sacchetti, B., Ambroqi Lorenzini, C., Baldi, E., Tassoni, G., Bucherelli, C., 1999. Memorization of contextual and CS conditioned fear response (freezing) in a one-trial acquisition paradigm. Arch Ital Biol 137, 235-248.

Sacchetti, B., Baldi, E., Lorenzini, C. A., Bucherelli, C., 2002. Cerebellar role in fear-conditioning consolidation. Proc Natl Acad Sci U S A 99, 8406-8411.

Sakai, K., Takahashi, K., Anaclet, C., Lin, J. S., 2010. Sleep-waking discharge of ventral tuberomammillary neurons in wild-type and histidine decarboxylase knock-out mice. Front Behav Neurosci 4, 53.

Sakamoto, K., Karelina, K., Obrietan, K., 2011. CREB: a multifaceted regulator of neuronal plasticity and protection. J Neurochem 116, 1-9. 
Sakata, T., Fukagawa, K., Fujimoto, K., Yoshimatsu, H., Shiraishi, T., Wada, H., 1988a. Feeding induced by blockade of histamine H1-receptor in rat brain. Experientia 44, 216-218.

Sakata, T., Ookuma, K., Fukagawa, K., Fujimoto, K., Yoshimatsu, H., Shiraishi, T., Wada, H., 1988b. Blockade of the histamine H1-receptor in the rat ventromedial hypothalamus and feeding elicitation. Brain Res 441, 403-407.

Sakata, T., Yoshimatsu, H., Masaki, T., Tsuda, K., 2003. Anti-obesity actions of mastication driven by histamine neurons in rats. Exp Biol Med (Maywood) 228, 1106-1110.

Sandi, C., 1998. The role and mechanisms of action of glucocorticoid involvement in memory storage. Neural Plast 6, 41-52.

Sandi, C., 2004. Stress, cognitive impairment and cell adhesion molecules. Nat Rev Neurosci 5, 917-930.

Sandi, C., Haller, J., 2015. Stress and the social brain: behavioural effects and neurobiological mechanisms. Nat Rev Neurosci 16, 290-304.

Sandi, C., Loscertales, M., 1999. Opposite effects on NCAM expression in the rat frontal cortex induced by acute vs. chronic corticosterone treatments. Brain Res 828, 127-134.

Sandi, C., Pinelo-Nava, M. T., 2007. Stress and memory: behavioral effects and neurobiological mechanisms. Neural Plast 2007, 78970.

Santarelli, L., Saxe, M., Gross, C., Surget, A., Battaglia, F., Dulawa, S., Weisstaub, N., Lee, J., Duman, R., Arancio, O., Belzung, C., Hen, R., 2003. Requirement of hippocampal neurogenesis for the behavioral effects of antidepressants. Science 301, 805-809.

Saper, C. B., Fuller, P. M., Pedersen, N. P., Lu, J., Scammell, T. E., 2010. Sleep state switching. Neuron 68, 1023-1042.

Sapolsky, R. M., 1996. Why stress is bad for your brain. Science 273, 749-750.

Sapolsky, R. M., 2000. Stress hormones: good and bad. Neurobiol Dis 7, 540-542.

Sapolsky, R. M., 2005. The influence of social hierarchy on primate health. Science 308, 648-652.

Scammell, T. E., Estabrooke, I. V., McCarthy, M. T., Chemelli, R. M., Yanagisawa, M., Miller, M. S., Saper, C. B., 2000. Hypothalamic arousal regions are activated during modafinil-induced wakefulness. J Neurosci 20, 8620-8628.

Schaefer, C., Enning, F., Mueller, J. K., Bumb, J. M., Rohleder, C., Odorfer, T. M., Klosterkötter, J., Hellmich, M., Koethe, D., Schmahl, C., Bohus, M., Leweke, F. M., 2014. Fatty acid ethanolamide levels are altered in borderline personality and complex posttraumatic stress disorders. Eur Arch Psychiatry Clin Neurosci 264, 459-463.

Schmauss, C., 2003. Serotonin 2C receptors: suicide, serotonin, and runaway RNA editing. Neuroscientist 9, 237-242.

Schwartz, G. J., 2011. Gut fat sensing in the negative feedback control of energy balance-recent advances. Physiol Behav 104, 621-623.

Schwartz, G. J., Fu, J., Astarita, G., Li, X., Gaetani, S., Campolongo, P., Cuomo, V., Piomelli, D., 2008. The lipid messenger OEA links dietary fat intake to satiety. Cell Metab 8, 281-288.

Schwartz, J. C., Arrang, J. M., Garbarg, M., Pollard, H., Ruat, M., 1991. Histaminergic transmission in the mammalian brain. Physiol Rev 71, 1-51. 
Histaminergic neurotransmission as a gateway for the effects of Oleoylethanolamide

Seeman, P., Schwarz, J., Chen, J. F., Szechtman, H., Perreault, M., McKnight, G. S., Roder, J. C., Quirion, R., Boksa, P., Srivastava, L. K., Yanai, K., Weinshenker, D., Sumiyoshi, T., 2006. Psychosis pathways converge via D2high dopamine receptors. Synapse 60, 319-346.

Seifert, R., Hagelüken, A., Höer, A., Höer, D., Grünbaum, L., Offermanns, S., Schwaner, I., Zingel, V., Schunack, W., Schultz, G., 1994. The H1 receptor agonist 2-(3-chlorophenyl)histamine activates Gi proteins in HL-60 cells through a mechanism that is independent of known histamine receptor subtypes. Mol Pharmacol 45, 578-586.

Selbach, O., Brown, R. E., Haas, H. L., 1997. Long-term increase of hippocampal excitability by histamine and cyclic AMP. Neuropharmacology 36, 1539-1548.

Seligman, M. E., Beagley, G., 1975. Learned helplessness in the rat. J Comp Physiol Psychol 88, 534-541.

Seligman, M. E., Maier, S. F., 1967. Failure to escape traumatic shock. J Exp Psychol 74, 1-9.

Selten, J. P., Cantor-Graae, E., 2005. Social defeat: risk factor for schizophrenia? Br J Psychiatry 187, 101-102.

Selten, J. P., Cantor-Graae, E., 2007. Hypothesis: social defeat is a risk factor for schizophrenia? Br J Psychiatry Suppl 51, s9-12.

Selten, J. P., van der Ven, E., Rutten, B. P., Cantor-Graae, E., 2013. The social defeat hypothesis of schizophrenia: an update. Schizophr Bull 39, 1180-1186.

Sergeeva, O. A., Amberger, B. T., Haas, H. L., 2007. Editing of AMPA and serotonin 2C receptors in individual central neurons, controlling wakefulness. Cell Mol Neurobiol 27, 669-680.

Sgoifo, A., Koolhaas, J., De Boer, S., Musso, E., Stilli, D., Buwalda, B., Meerlo, P., 1999. Social stress, autonomic neural activation, and cardiac activity in rats. Neurosci Biobehav Rev 23, 915-923.

Shan, L., Bao, A. M., Swaab, D. F., 2015a. The human histaminergic system in neuropsychiatric disorders. Trends Neurosci 38, 167-177.

Shan, L., Bossers, K., Unmehopa, U., Bao, A. M., Swaab, D. F., 2012. Alterations in the histaminergic system in Alzheimer's disease: a postmortem study. Neurobiol Aging 33, 2585-2598.

Shan, L., Dauvilliers, Y., Siegel, J. M., 2015b. Interactions of the histamine and hypocretin systems in CNS disorders. Nat Rev Neurol 11, 401-413.

Sheiner, J. B., Morris, P., Anderson, G. H., 1985. Food intake suppression by histidine. Pharmacol Biochem Behav 23, 721-726.

Sherin, J. E., Elmquist, J. K., Torrealba, F., Saper, C. B., 1998. Innervation of histaminergic tuberomammillary neurons by GABAergic and galaninergic neurons in the ventrolateral preoptic nucleus of the rat. J Neurosci 18, 4705-4721.

Shimozuru, M., Kikusui, T., Takeuchi, Y., Mori, Y., 2008. Effects of isolation-rearing on the development of social behaviors in male Mongolian gerbils (Meriones unguiculatus). Physiol Behav 94, 491-500.

Shors, T. J., 2004. Learning during stressful times. Learn Mem 11, 137-144.

Siuciak, J. A., Lewis, D. R., Wiegand, S. J., Lindsay, R. M., 1997. Antidepressant-like effect of brain-derived neurotrophic factor (BDNF). Pharmacol Biochem Behav 56, 131-137. 
Smith, M. A., 1996. Hippocampal vulnerability to stress and aging: possible role of neurotrophic factors. Behav Brain Res 78, 25-36.

Song, C., Earley, B., Leonard, B. E., 1996. Behavioural and immunological effects of the antihistamine terfenadine in olfactory bulbectomized rats. Eur Neuropsychopharmacol $6,157-162$.

Sousa, N., Lukoyanov, N. V., Madeira, M. D., Almeida, O. F., Paula-Barbosa, M. M., 2000. Reorganization of the morphology of hippocampal neurites and synapses after stressinduced damage correlates with behavioral improvement. Neuroscience 97, 253-266.

Southwick, S. M., Vythilingam, M., Charney, D. S., 2005. The psychobiology of depression and resilience to stress: implications for prevention and treatment. Annu Rev Clin Psychol 1, 255-291.

Squire, L. R., Wixted, J. T., Clark, R. E., 2007. Recognition memory and the medial temporal lobe: a new perspective. Nat Rev Neurosci 8, 872-883.

Stahl, S. M., 2008. Stahl's essential psychopharmacology : neuroscientific basis and practical applications. . CAMBRIDGE UNIVERSITY PRESS

Steinman, L., 2004. Elaborate interactions between the immune and nervous systems. Nat Immunol 5, 575-581.

Steru, L., Chermat, R., Thierry, B., Simon, P., 1985. The tail suspension test: a new method for screening antidepressants in mice. Psychopharmacology (Berl) 85, 367-370.

Stevens, D. R., Kuramasu, A., Eriksson, K. S., Selbach, O., Haas, H. L., 2004. Alpha 2-adrenergic receptor-mediated presynaptic inhibition of GABAergic IPSPs in rat histaminergic neurons. Neuropharmacology 46, 1018-1022.

Strakhova, M. I., Nikkel, A. L., Manelli, A. M., Hsieh, G. C., Esbenshade, T. A., Brioni, J. D., Bitner, R. S., 2009. Localization of histamine H4 receptors in the central nervous system of human and rat. Brain Res 1250, 41-48.

Stratakis, C. A., Chrousos, G. P., 1995. Neuroendocrinology and pathophysiology of the stress system. Ann N Y Acad Sci 771, 1-18.

Suardíaz, M., Estivill-Torrús, G., Goicoechea, C., Bilbao, A., Rodríguez de Fonseca, F., 2007. Analgesic properties of oleoylethanolamide (OEA) in visceral and inflammatory pain. Pain $133,99-110$.

Swaab, D. F., Lucassen, P. J., Salehi, A., Scherder, E. J., van Someren, E. J., Verwer, R. W., 1998. Reduced neuronal activity and reactivation in Alzheimer's disease. Prog Brain Res 117, 343-377.

Takagi, H., Morishima, Y., Matsuyama, T., Hayashi, H., Watanabe, T., Wada, H., 1986. Histaminergic axons in the neostriatum and cerebral cortex of the rat: a correlated light and electron microscopic immunocytochemical study using histidine decarboxylase as a marker. Brain Res 364, 114-123.

Takahashi, A., Arielle, N., Schilit, J., Kim, J., DeBold, T., Koide, K., Miczek, 2012. Behavioral characterization of escalated aggression induced by GABA B receptor activation in the dorsal raphe nucleus. Psychopharmacology, Springer-Verlag, pp. 155-166.

Takahashi, K., Lin, J. S., Sakai, K., 2006. Neuronal activity of histaminergic tuberomammillary neurons during wake-sleep states in the mouse. J Neurosci 26, 10292-10298.

Takeda, N., Inagaki, S., Shiosaka, S., Taguchi, Y., Oertel, W. H., Tohyama, M., Watanabe, T., Wada, H., 1984. Immunohistochemical evidence for the coexistence of histidine decarboxylase-like and glutamate decarboxylase-like immunoreactivities in nerve cells 
Histaminergic neurotransmission as a gateway for the effects of Oleoylethanolamide

of the magnocellular nucleus of the posterior hypothalamus of rats. Proc Natl Acad Sci U S A 81, 7647-7650.

Tardito, D., Perez, J., Tiraboschi, E., Musazzi, L., Racagni, G., Popoli, M., 2006. Signaling pathways regulating gene expression, neuroplasticity, and neurotrophic mechanisms in the action of antidepressants: a critical overview. Pharmacol Rev 58, 115-134.

Taylor, K. M., Snyder, S. H., 1971. Brain histamine: rapid apparent turnover altered by restraint and cold stress. Science 172, 1037-1039.

Teuscher, C., Poynter, M. E., Offner, H., Zamora, A., Watanabe, T., Fillmore, P. D., Zachary, J. F., Blankenhorn, E. P., 2004. Attenuation of Th1 effector cell responses and susceptibility to experimental allergic encephalomyelitis in histamine $\mathrm{H} 2$ receptor knockout mice is due to dysregulation of cytokine production by antigen-presenting cells. Am J Pathol 164, 883-892.

Teuscher, C., Subramanian, M., Noubade, R., Gao, J. F., Offner, H., Zachary, J. F., Blankenhorn, E. P., 2007. Central histamine $\mathrm{H} 3$ receptor signaling negatively regulates susceptibility to autoimmune inflammatory disease of the CNS. Proc Natl Acad Sci U S A 104, 10146-10151.

Theoharides, T. C., Konstantinidou, A. D., 2007. Corticotropin-releasing hormone and the blood-brain-barrier. Front Biosci 12, 1615-1628.

Thierry, B., Stéru, L., Simon, P., Porsolt, R. D., 1986. The tail suspension test: ethical considerations. Psychopharmacology (Berl) 90, 284-285.

Thoenen, H., 1995. Neurotrophins and neuronal plasticity. Science 270, 593-598.

Tiligada, E., Kyriakidis, K., Chazot, P. L., Passani, M. B., 2011. Histamine pharmacology and new CNS drug targets. CNS Neurosci Ther 17, 620-628.

Tiligada, E., Zampeli, E., Sander, K., Stark, H., 2009. Histamine H3 and H4 receptors as novel drug targets. Expert Opin Investig Drugs 18, 1519-1531.

Timonen, M., Jokelainen, J., Hakko, H., Silvennoinen-Kassinen, S., Meyer-Rochow, V. B., Herva, A., Räsänen, P., 2003. Atopy and depression: results from the Northern Finland 1966 Birth Cohort Study. Mol Psychiatry 8, 738-744.

Toftegaard, C. L., Knigge, U., Kjaer, A., Warberg, J., 2003. The role of hypothalamic histamine in leptin-induced suppression of short-term food intake in fasted rats. Regul Pept 111, 83-90.

Tokita, S., Takahashi, K., Kotani, H., 2006. Recent advances in molecular pharmacology of the histamine systems: physiology and pharmacology of histamine $\mathrm{H} 3$ receptor: roles in feeding regulation and therapeutic potential for metabolic disorders. J Pharmacol Sci 101, 12-18.

Toyota, H., Dugovic, C., Koehl, M., Laposky, A. D., Weber, C., Ngo, K., Wu, Y., Lee, D. H., Yanai, K., Sakurai, E., Watanabe, T., Liu, C., Chen, J., Barbier, A. J., Turek, F. W., FungLeung, W. P., Lovenberg, T. W., 2002. Behavioral characterization of mice lacking histamine H(3) receptors. Mol Pharmacol 62, 389-397.

Traiffort, E., Pollard, H., Moreau, J., Ruat, M., Schwartz, J. C., Martinez-Mir, M. I., Palacios, J. M., 1992. Pharmacological characterization and autoradiographic localization of histamine $\mathrm{H} 2$ receptors in human brain identified with [125I]iodoaminopotentidine. J Neurochem 59, 290-299.

Tramullas, M., Dinan, T. G., Cryan, J. F., 2012. Chronic psychosocial stress induces visceral hyperalgesia in mice. Stress 15, 281-292. 
Trifilieff, P., Calandreau, L., Herry, C., Mons, N., Micheau, J., 2007. Biphasic ERK1/2 activation in both the hippocampus and amygdala may reveal a system consolidation of contextual fear memory. Neurobiol Learn Mem 88, 424-434.

Trivedi, M. H., Fava, M., Marangell, L. B., Osser, D. N., Shelton, R. C., 2006. Use of treatment algorithms for depression. J Clin Psychiatry 67, 1458-1465.

Turner, B. A., Sparrow, J., Cai, B., Monroe, J., Mikawa, T., Hempstead, B. L., 2006. TrkB/ BDNF signaling regulates photoreceptor progenitor cell fate decisions. Dev Biol 299, 455-465.

Urani, A., Chourbaji, S., Gass, P., 2005. Mutant mouse models of depression: candidate genes and current mouse lines. Neurosci Biobehav Rev 29, 805-828.

Uygun, D. S., Ye, Z., Zecharia, A. Y., Harding, E. C., Yu, X., Yustos, R., Vyssotski, A. L., Brickley, S. G., Franks, N. P., Wisden, W., 2016. Bottom-Up versus Top-Down Induction of Sleep by Zolpidem Acting on Histaminergic and Neocortex Neurons. J Neurosci 36, 11171-11184.

Valzelli, L., 1973. The "isolation syndrome" in mice. Psychopharmacologia 31, 305-320.

van Bokhoven, H., 2011. Genetic and epigenetic networks in intellectual disabilities. Annu Rev Genet 45, 81-104.

Van den Berg, C. L., Pijlman, F. T., Koning, H. A., Diergaarde, L., Van Ree, J. M., Spruijt, B. M., 1999. Isolation changes the incentive value of sucrose and social behaviour in juvenile and adult rats. Behav Brain Res 106, 133-142.

van der Kooij, M. A., Fantin, M., Kraev, I., Korshunova, I., Grosse, J., Zanoletti, O., Guirado, R., Garcia-Mompó, C., Nacher, J., Stewart, M. G., Berezin, V., Sandi, C., 2014. Impaired hippocampal neuroligin-2 function by chronic stress or synthetic peptide treatment is linked to social deficits and increased aggression. Neuropsychopharmacology 39, 1148-1158.

Vanni-Mercier, G., Gigout, S., Debilly, G., Lin, J. S., 2003. Waking selective neurons in the posterior hypothalamus and their response to histamine H3-receptor ligands: an electrophysiological study in freely moving cats. Behav Brain Res 144, 227-241.

Veena, J., Srikumar, B. N., Mahati, K., Bhagya, V., Raju, T. R., Shankaranarayana Rao, B. S., 2009. Enriched environment restores hippocampal cell proliferation and ameliorates cognitive deficits in chronically stressed rats. J Neurosci Res 87, 831-843.

Verdière, M., Rose, C., Schwartz, J. C., 1977. Turnover of cerebral histamine in a stressful situation. Brain Res 129, 107-119.

Vertes, R. P., 2004. Differential projections of the infralimbic and prelimbic cortex in the rat. Synapse 51, 32-58.

Victor, M., 1987. The irrelevance of mammillary body lesions in the causation of the Korsakoff amnesic state. Int J Neurol 21-22, 51-57.

Vidal, J., Buwalda, B., Koolhaas, J. M., 2011. Male Wistar rats are more susceptible to lasting social anxiety than Wild-type Groningen rats following social defeat stress during adolescence. Behav Processes 88, 76-80.

Vollmayr, B., Bachteler, D., Vengeliene, V., Gass, P., Spanagel, R., Henn, F., 2004. Rats with congenital learned helplessness respond less to sucrose but show no deficits in activity or learning. Behav Brain Res 150, 217-221.

Vollmayr, B., Mahlstedt, M. M., Henn, F. A., 2007. Neurogenesis and depression: what animal models tell us about the link. Eur Arch Psychiatry Clin Neurosci 257, 300-303. 
Histaminergic neurotransmission as a gateway for the effects of Oleoylethanolamide

von Coburg, Y., Kottke, T., Weizel, L., Ligneau, X., Stark, H., 2009. Potential utility of histamine $\mathrm{H} 3$ receptor antagonist pharmacophore in antipsychotics. Bioorg Med Chem Lett 19, 538-542.

von Holst, D., 1998. The Concept of Stress and Its Relevance for Animal Behavior. Academic Press, pp. 1-131.

Vyas, A., Mitra, R., Shankaranarayana Rao, B. S., Chattarji, S., 2002. Chronic stress induces contrasting patterns of dendritic remodeling in hippocampal and amygdaloid neurons. J Neurosci 22, 6810-6818.

Vyas, A., Pillai, A. G., Chattarji, S., 2004. Recovery after chronic stress fails to reverse amygdaloid neuronal hypertrophy and enhanced anxiety-like behavior. Neuroscience 128, 667-673.

Wada, H., Inagaki, N., Itowi, N., Yamatodani, A., 1991. Histaminergic neuron system in the brain: distribution and possible functions. Brain Res Bull 27, 367-370.

Walker, E. R., McGee, R. E., Druss, B. G., 2015. Mortality in mental disorders and global disease burden implications: a systematic review and meta-analysis. JAMA Psychiatry $72,334-341$.

Wallace, T. L., Stellitano, K. E., Neve, R. L., Duman, R. S., 2004. Effects of cyclic adenosine monophosphate response element binding protein overexpression in the basolateral amygdala on behavioral models of depression and anxiety. Biol Psychiatry 56, 151-160.

Wallis, J. D., 2011. Cross-species studies of orbitofrontal cortex and value-based decisionmaking. Nat Neurosci 15, 13-19.

Wang, H., Zhang, Y., Qiao, M., 2013. Mechanisms of extracellular signal-regulated kinase/cAMP response element-binding protein/brain-derived neurotrophic factor signal transduction pathway in depressive disorder. Neural Regen Res 8, 843-852.

Wang, X., Miyares, R. L., Ahern, G. P., 2005. Oleoylethanolamide excites vagal sensory neurones, induces visceral pain and reduces short-term food intake in mice via capsaicin receptor TRPV1. J Physiol 564, 541-547.

Wang, Y. X., Kotlikoff, M. I., 2000. Signalling pathway for histamine activation of non-selective cation channels in equine tracheal myocytes. J Physiol $523 \mathrm{Pt}$ 1, 131-138.

Warburton, E. C., Brown, M. W., 2010. Findings from animals concerning when interactions between perirhinal cortex, hippocampus and medial prefrontal cortex are necessary for recognition memory. Neuropsychologia 48, 2262-2272.

Warburton, E. C., Brown, M. W., 2015. Neural circuitry for rat recognition memory. Behav Brain Res 285, 131-139.

Warren, D.A, Castro, C. A., Rudy, J. W., al., e., 1991. Psychobiology p. 127.

Watanabe, T., Taguchi, Y., Shiosaka, S., Tanaka, J., Kubota, H., Terano, Y., Tohyama, M., Wada, H., 1984. Distribution of the histaminergic neuron system in the central nervous system of rats; a fluorescent immunohistochemical analysis with histidine decarboxylase as a marker. Brain Res 295, 13-25.

Wehner, J. M., Radcliffe, R. A., 2004. Cued and contextual fear conditioning in mice. Curr Protoc Neurosci Chapter 8, Unit 8.5C.

Wei, B., Tai, F., Liu, X., Ma, L., Yang, X., Jia, R., Zhang, X., 2013. Neonatal tactile stimulation alleviates the negative effects of neonatal isolation on novel object recognition, sociability and neuroendocrine levels in male adult mandarin voles (Microtus mandarinus). Physiol Behav 112-113, 14-22. 
Weiss, J., Kilts, C., 1998. Animal models of depression, schizophrenia. In: Nemeroff, CB, Schatzberg AF American Psychiatric Press, Textbook of Psychopharmacology.

Whitcup, S. M., Bradford, R., Lue, J., Schiffman, R. M., Abelson, M. B., 2004. Efficacy and tolerability of ophthalmic epinastine: a randomized, double-masked, parallel-group, active- and vehicle-controlled environmental trial in patients with seasonal allergic conjunctivitis. Clin Ther 26, 29-34.

WHITE, T., 1959. Formation and catabolism of histamine in brain tissue in vitro. J Physiol $149,34-42$.

Whiteford, H. A., Degenhardt, L., Rehm, J., Baxter, A. J., Ferrari, A. J., Erskine, H. E., Charlson, F. J., Norman, R. E., Flaxman, A. D., Johns, N., Burstein, R., Murray, C. J., Vos, T., 2013. Global burden of disease attributable to mental and substance use disorders: findings from the Global Burden of Disease Study 2010. Lancet 382, 1575-1586.

Williams, R. H., Chee, M. J., Kroeger, D., Ferrari, L. L., Maratos-Flier, E., Scammell, T. E., Arrigoni, E., 2014. Optogenetic-mediated release of histamine reveals distal and autoregulatory mechanisms for controlling arousal. J Neurosci 34, 6023-6029.

Willner, P., 1990. Animal models of depression: an overview. Pharmacol Ther 45, 425-455.

Willner, P., 1997. Validity, reliability and utility of the chronic mild stress model of depression: a 10-year review and evaluation. Psychopharmacology (Berl) 134, 319-329.

Willner, P., Mitchell, P. J., 2002. The validity of animal models of predisposition to depression. Behav Pharmacol 13, 169-188.

Willner, P., Muscat, R., Papp, M., 1992. Chronic mild stress-induced anhedonia: a realistic animal model of depression. Neurosci Biobehav Rev 16, 525-534.

Willner, P., Towell, A., Sampson, D., Sophokleous, S., Muscat, R., 1987. Reduction of sucrose preference by chronic unpredictable mild stress, and its restoration by a tricyclic antidepressant. Psychopharmacology (Berl) 93, 358-364.

Winters, B. D., Saksida, L. M., Bussey, T. J., 2008. Object recognition memory: neurobiological mechanisms of encoding, consolidation and retrieval. Neurosci Biobehav Rev 32, 1055-1070.

Wommack, J. C., Salinas, A., Melloni, R. H., Delville, Y., 2004. Behavioural and neuroendocrine adaptations to repeated stress during puberty in male golden hamsters. J Neuroendocrinol 16, 767-775.

Woo, N. H., Teng, H. K., Siao, C. J., Chiaruttini, C., Pang, P. T., Milner, T. A., Hempstead, B. L., Lu, B., 2005. Activation of p75NTR by proBDNF facilitates hippocampal long-term depression. Nat Neurosci 8, 1069-1077.

Wood, G. E., Young, L. T., Reagan, L. P., McEwen, B. S., 2003. Acute and chronic restraint stress alter the incidence of social conflict in male rats. Horm Behav 43, 205-213.

Workman, J. L., Fonken, L. K., Gusfa, J., Kassouf, K. M., Nelson, R. J., 2011. Post-weaning environmental enrichment alters affective responses and interacts with behavioral testing to alter nNOS immunoreactivity. Pharmacol Biochem Behav 100, 25-32.

Yamakami, J., Sakurai, E., Kuramasu, A., Yanai, K., Watanabe, T., Tanaka, Y., 2000. L-Histidine decarboxylase protein and activity in rat brain microvascular endothelial cells. Inflamm Res 49, 231-235.

Yamamoto, Y., Mikami, A., Fujii, Y., Kamei, C., 2007. Effect of histamine on muscimolinduced working memory deficits in radial maze performance. J Pharmacol Sci 104, 252-257. 
Yamamoto, Y., Mochizuki, T., Okakura-Mochizuki, K., Uno, A., Yamatodani, A., 1997. Thioperamide, a histamine $\mathrm{H} 3$ receptor antagonist, increases GABA release from the rat hypothalamus. Methods Find Exp Clin Pharmacol 19, 289-298.

Yanai, K., Tashiro, M., 2007. The physiological and pathophysiological roles of neuronal histamine: an insight from human positron emission tomography studies. Pharmacol Ther 113, 1-15.

Yang, J., Siao, C. J., Nagappan, G., Marinic, T., Jing, D., McGrath, K., Chen, Z. Y., Mark, W., Tessarollo, L., Lee, F. S., Lu, B., Hempstead, B. L., 2009. Neuronal release of proBDNF. Nat Neurosci 12, 113-115.

Yang, Y. W., Tseng, K. C., Chen, Y. H., Yang, J. Y., 2010. Associations among eczema, asthma, serum immunoglobulin $\mathrm{E}$ and depression in adults: a population-based study. Allergy 65, 801-802.

Yohe, L. R., Suzuki, H., Lucas, L. R., 2012. Aggression is suppressed by acute stress but induced by chronic stress: immobilization effects on aggression, hormones, and cortical 5-HT(1B)/ striatal dopamine $\mathrm{D}(2)$ receptor density. Cogn Affect Behav Neurosci 12, 446-459.

Yoshimoto, R., Miyamoto, Y., Takahashi, K., Kotani, H., Kanatani, A., Tokita, S., 2006. Impaired drinking response in histamine $\mathrm{H} 3$ receptor knockout mice following dehydration or angiotensin-II challenge. Pharmacol Biochem Behav 84, 504-510.

Yu, H. L., Sun, L. P., Li, M. M., Quan, Z. S., 2015a. Involvement of norepinephrine and serotonin system in antidepressant-like effects of oleoylethanolamide in the mice models of behavior despair. Neurosci Lett 593, 24-28.

Yu, P., An, S., Tai, F., Wang, J., Wu, R., Wang, B., 2013. Early social deprivation impairs pair bonding and alters serum corticosterone and the NAcc dopamine system in mandarin voles. Psychoneuroendocrinology 38, 3128-3138.

Yu, X., Ye, Z., Houston, C. M., Zecharia, A. Y., Ma, Y., Zhang, Z., Uygun, D. S., Parker, S., Vyssotski, A. L., Yustos, R., Franks, N. P., Brickley, S. G., Wisden, W., 2015b. Wakefulness Is Governed by GABA and Histamine Cotransmission. Neuron 87, 164-178.

Zagrebelsky, M., Holz, A., Dechant, G., Barde, Y. A., Bonhoeffer, T., Korte, M., 2005. The p75 neurotrophin receptor negatively modulates dendrite complexity and spine density in hippocampal neurons. J Neurosci 25, 9989-9999.

Zhu, Y., Michalovich, D., Wu, H., Tan, K. B., Dytko, G. M., Mannan, I. J., Boyce, R., Alston, J., Tierney, L. A., Li, X., Herrity, N. C., Vawter, L., Sarau, H. M., Ames, R. S., Davenport, C. M., Hieble, J. P., Wilson, S., Bergsma, D. J., Fitzgerald, L. R., 2001. Cloning, expression, and pharmacological characterization of a novel human histamine receptor. Mol Pharmacol 59, 434-441. 


\section{Figures}

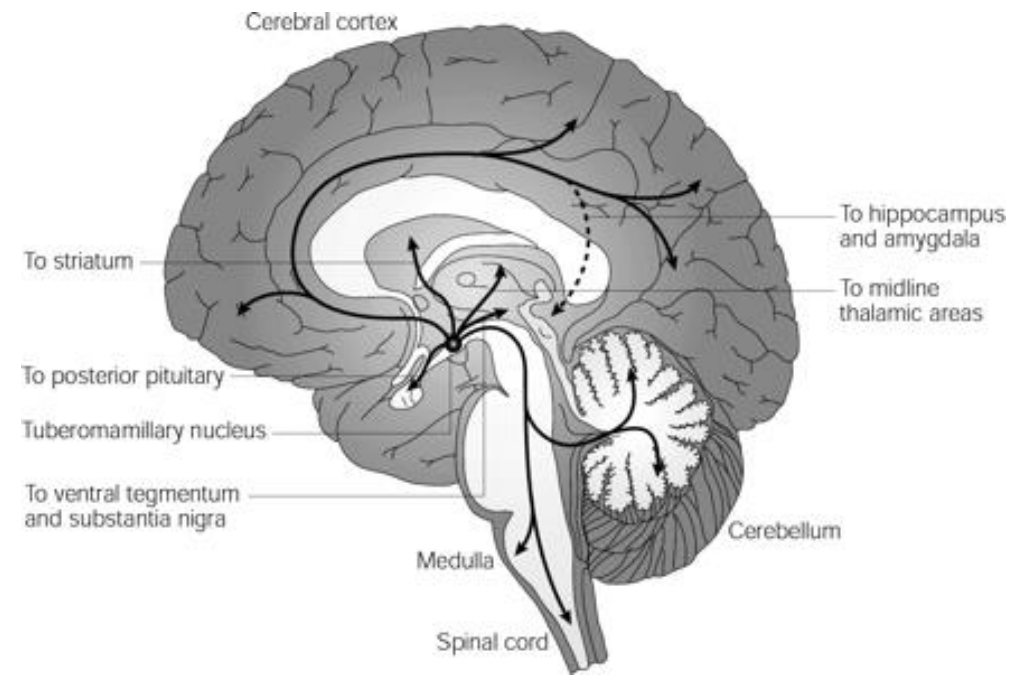

Figure 1. The histaminergic system in human brain. The histaminergic fibers emanating from the tuberomamillary nucleus project to and arborize in the whole central nervous system (Haas et al., 2008). 
Histaminergic neurotransmission as a gateway for the effects of Oleoylethanolamide

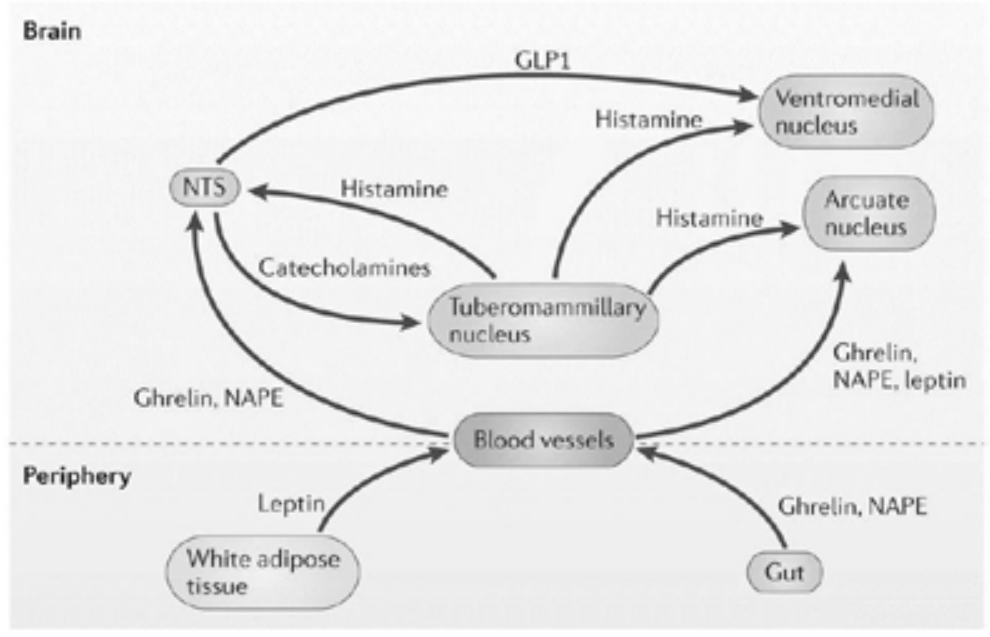

Nature Reviews | Neuroscience

Figure 2. Key brain areas involved in the regulation of feeding and their innervation by histaminergic fibres (Panula and Nuutinen, 2013). 


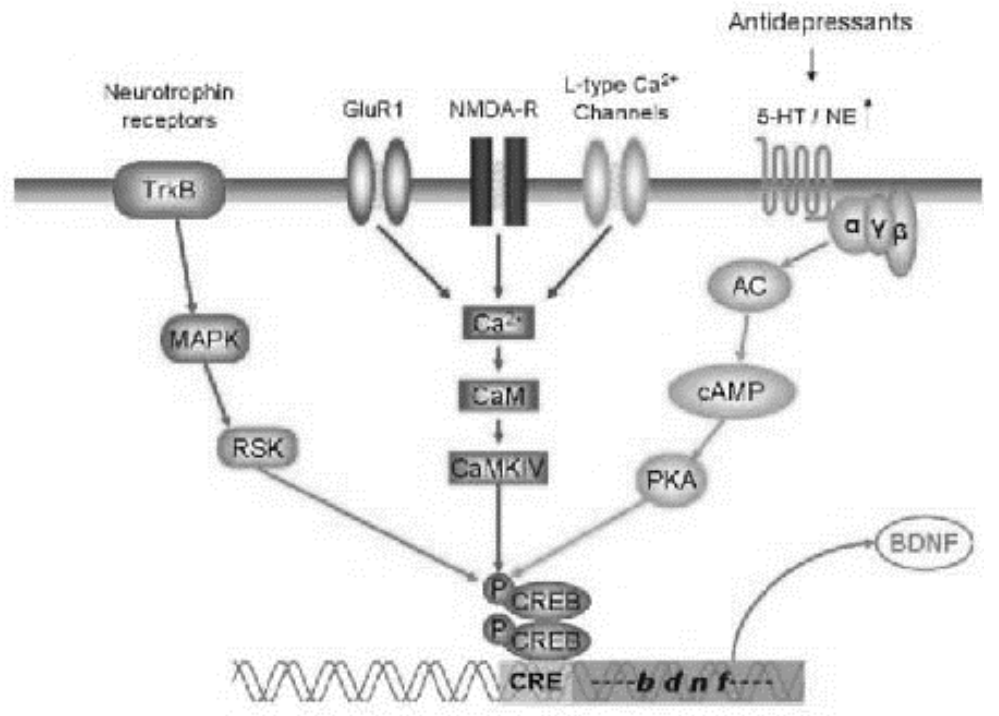

Figure 3. cAMP response element-binding protein/brain-derived neurotrophic factor signal transduction pathway (Wang et al., 2013).

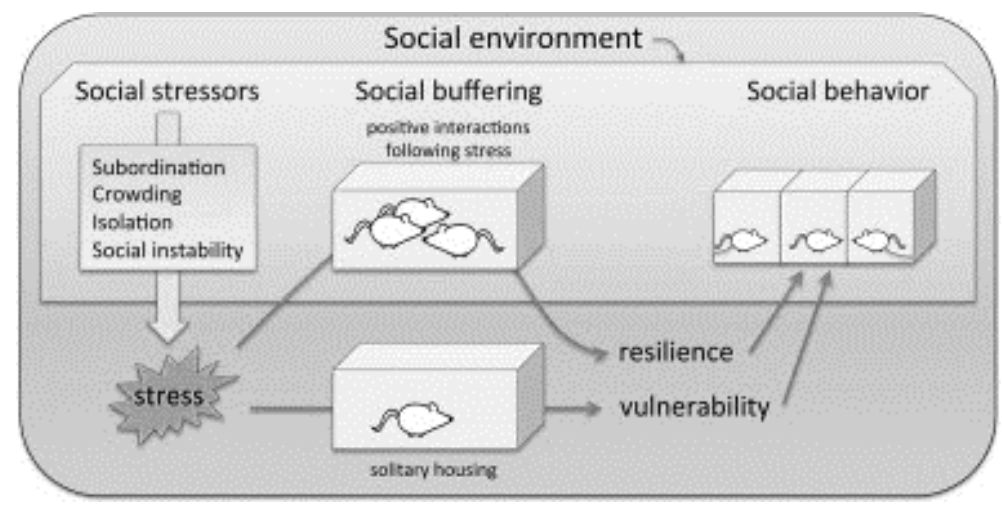

Figure 4. Schematic representation of the levels at which the social environment impacts and reflects the individual (Beery and Kaufer, 2015). 


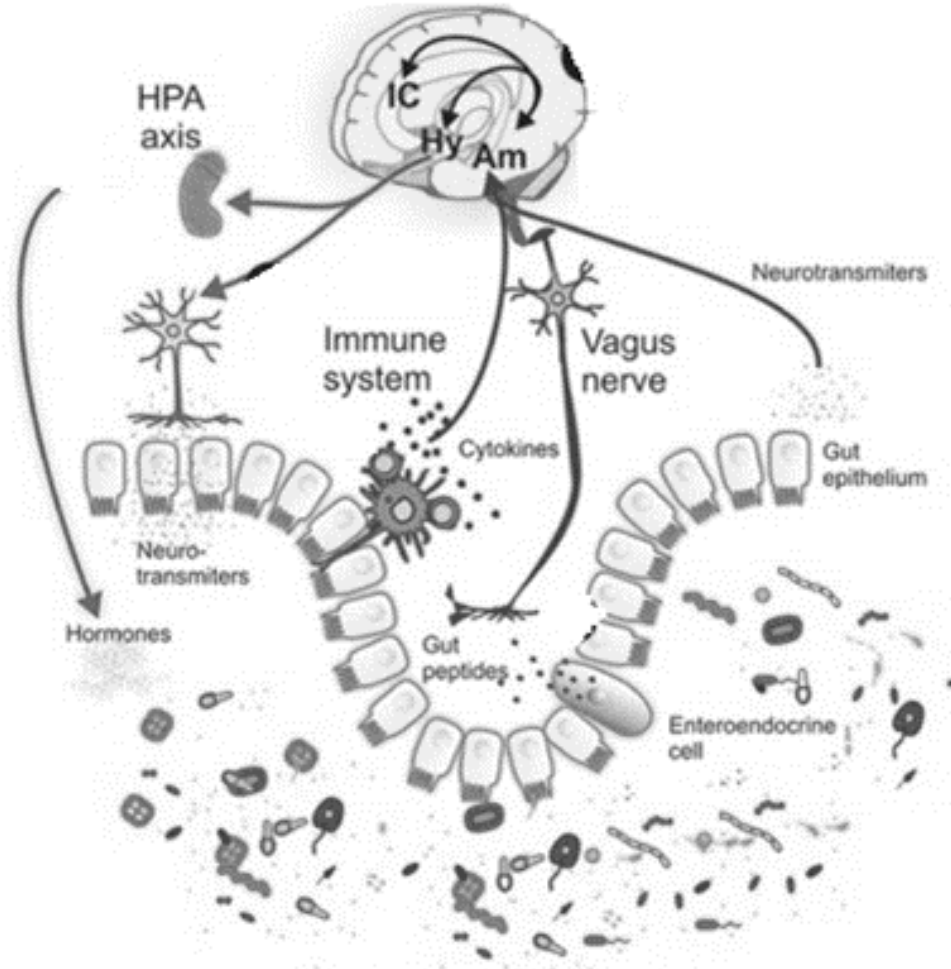

\section{Gut microbiota}

Figure 5. Microbiota-gut-brain (MGB) axis. Direct and indirect pathways support the bidirectional interactions between the gut microbiota and the central nervous system (CNS); involving endocrine, immune and neural pathways (Montiel-Castro et al., 2013). 

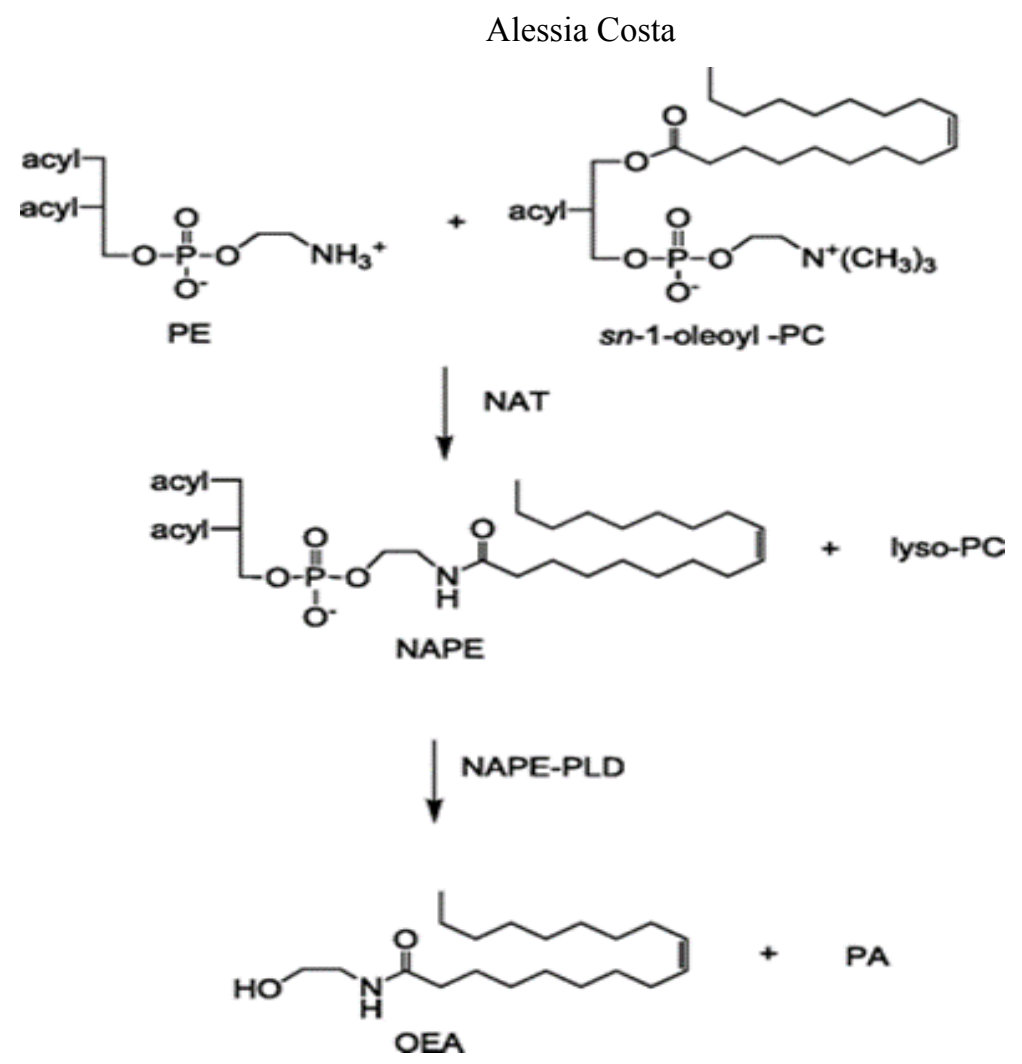

Figure 6. Biochemical pathway responsible for the production of OEA (Fu et al., 2011). 
Histaminergic neurotransmission as a gateway for the effects of Oleoylethanolamide

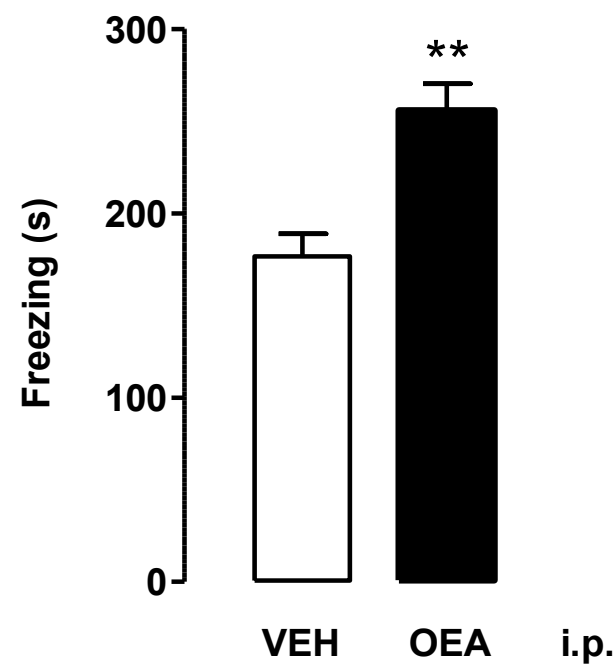

Figure 7. OEA $(10 \mathrm{mg} / \mathrm{kg})$ or vehicle (VEH) were injected i.p. 10 minutes after contextual fear conditioning. Fear retention was evaluated 72 hours after conditioning. Bars represent mean values \pm sem of 8 to 10 rats/group; $* * P<.01$; unpaired $t$ test. 


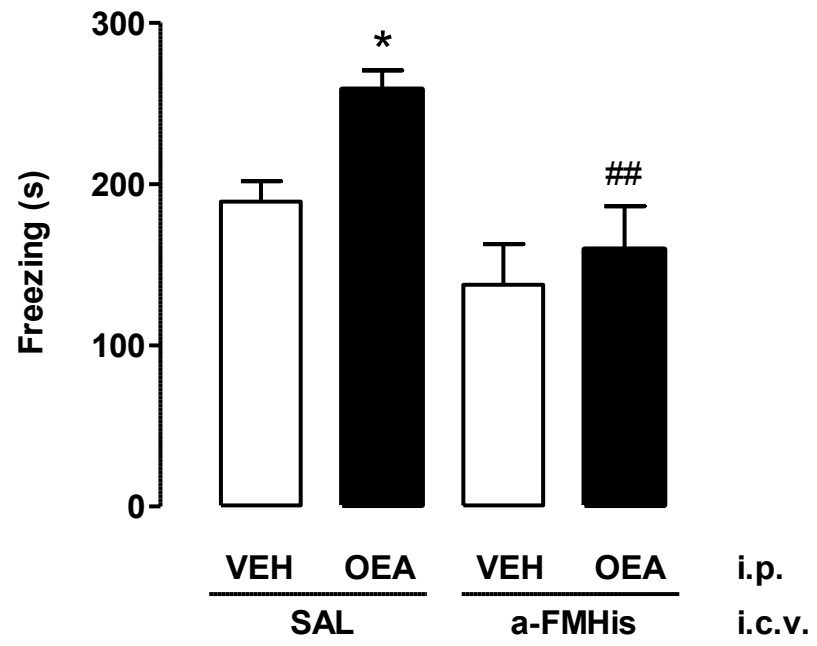

Figure 8. $\alpha$-FMHis or saline was infused i.c.v. 24 hours prior to fear conditioning. OEA $(10 \mathrm{mg} / \mathrm{kg})$ or vehicle (VEH) were injected i.p. 10 minutes after contextual fear conditioning. Fear retention was evaluated 72 hours after conditioning. Latencies of $\alpha$-FMHis groups did not significantly differ from controls. Data are expressed as means \pm SEM of 10 to 14 animals for each group; ANOVA and Newman-Keuls posthoc test, ${ }^{*} P<.05$ vs saline (SAL) controls. ${ }^{\#} P<.01$ vs OEA/SAL. 


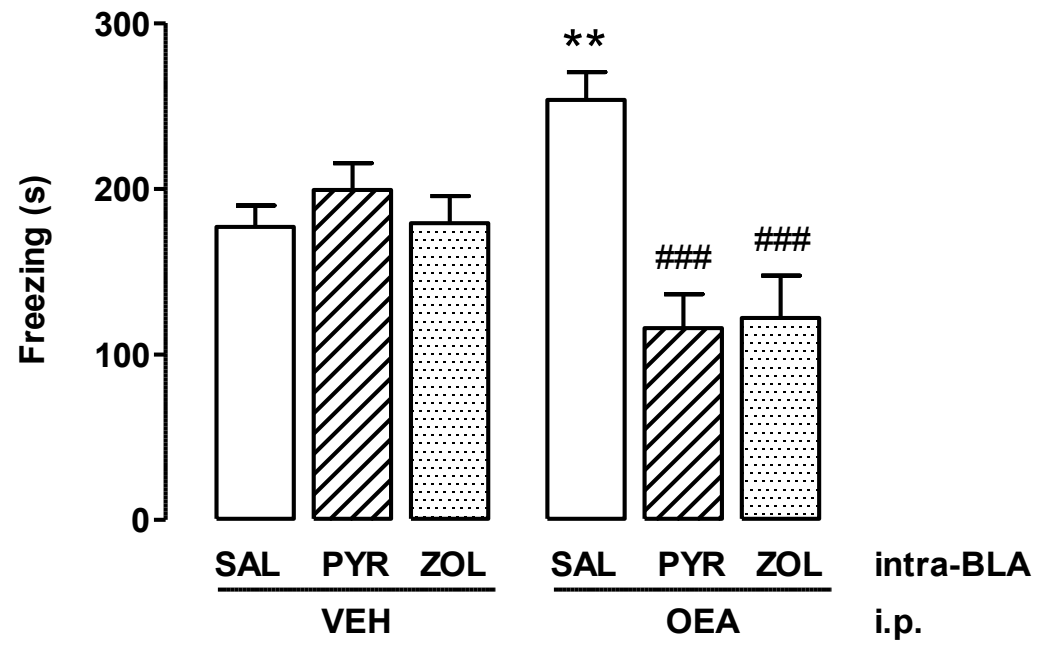

Figure 9. Rats received bilateral intra-BLA infusions of zolantidine (ZOL), pyrilamine (PYR), or saline, and OEA or vehicle i.p. immediately after training. Data are expressed as means \pm SEM of 9 to 14 animals for each group; ANOVA and NewmanKeuls' posthoc test, ${ }^{* *} P<.01$ vs SAL/VEH controls; ${ }^{\# \# P} P .001$ vs SAL/OEA. 

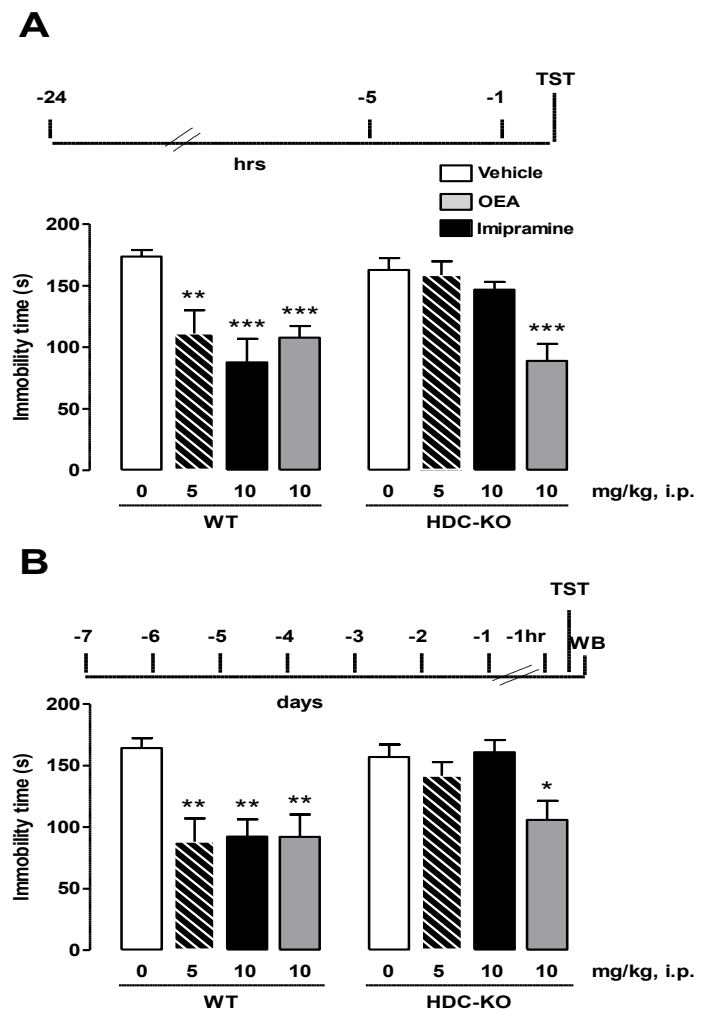

C

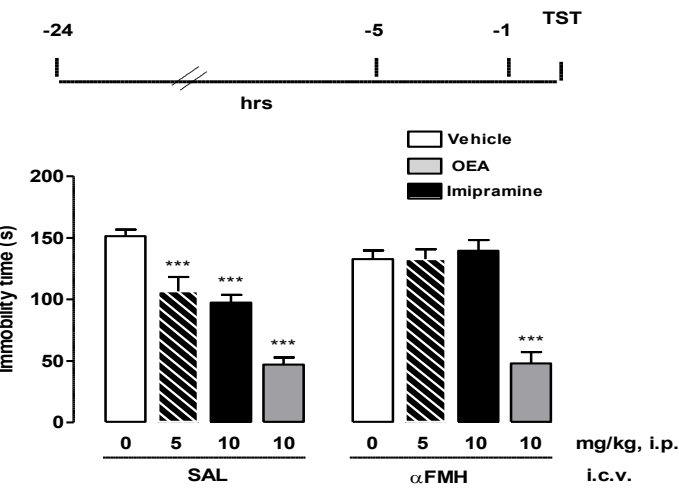

Figure 10. OEA induce antidepressant-like effects in normal but not in histaminedeficient mice in the Tail Suspension Test. (A) Effect of systemic administration of OEA (repeated regimen) or vehicle to WT and HDC-KO male mice $(n=5-12)$. (B) Effect of systemic administration of OEA $(10 \mathrm{mg} / \mathrm{kg}$ i.p. sub-chronic regimen) or vehicle to WT and HDC-KO male mice $(\mathrm{n}=5-10)$. (C) Effect of systemic administration of OEA in saline or $\alpha-F M H ~(5 \mu$ i.c.v. $)$ injected CD1 mice $(n=5-12)$. 
Histaminergic neurotransmission as a gateway for the effects of Oleoylethanolamide

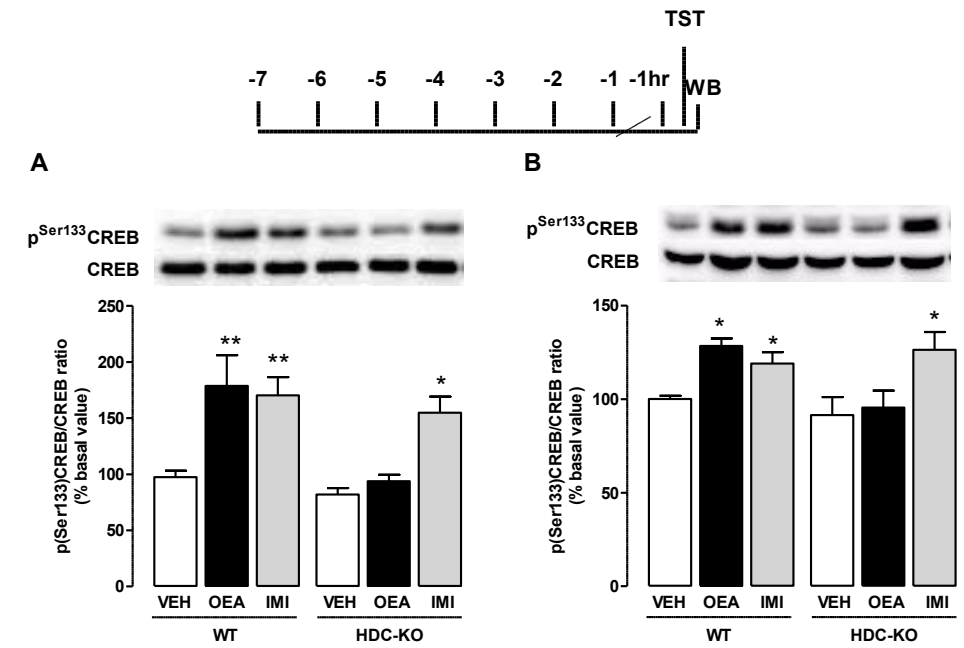

Figure 11. OEA chronic administration increased Ser133CREB phosphorylation in hippocampal (A) and cortical (B) homogenates from normal (WT) but not from histamine-deprived mice (HDC-KO) challenged in the tail suspension test. $(n=5$; $* \mathrm{p}<0,05 * * \mathrm{p}<0,01$ ANOVA and Bonferroni post hoc test).
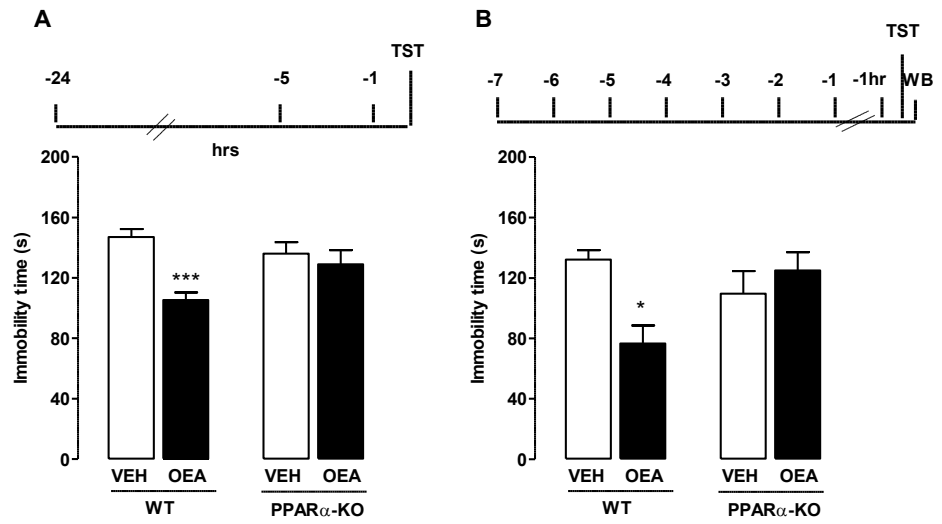

Figure 12. OEA induces antidepressant-like effects in normal but not in PPAR- $\alpha-K O$ mice in the Tail Suspension Test. (A) Effect of systemic administration of OEA (repeated regimen) or vehicle to WT and PPAR- $\alpha-K O$ male mice $(n=5-12)$. (B) Effect of systemic administration of OEA (10 mg/ $\mathrm{kg}$ i.p. sub-chronic regimen) or vehicle to WT and PPAR- $\alpha-K O$ male mice $(n=5-10) .(* * \mathrm{p}<0.01 * * * \mathrm{p}<0.001$ ANOVA and Bonferroni MCT). 
Alessia Costa

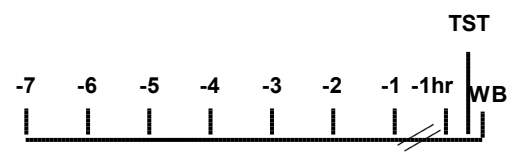

A

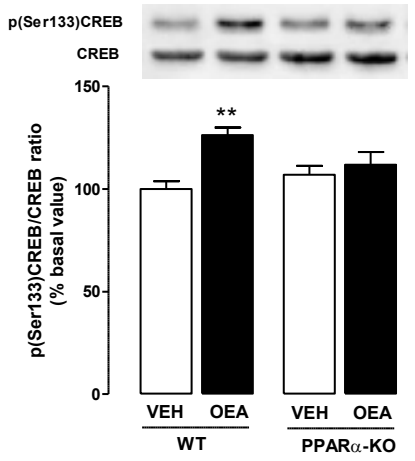

B

p(Ser133)CREB

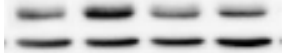

200

*

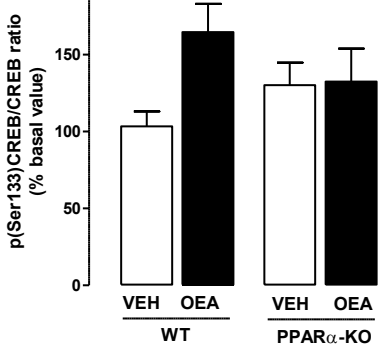

Figure 13. OEA chronic administration increased Ser133CREB phosphorylation in and hippocampal (A) cortical (B) homogenates from normal (WT) but not from PPAR- $\alpha$-deprived mice (PPAR- $\alpha \mathrm{KO}$ ) challenged in the tail suspension test. ( $\mathrm{n}=7-9$; $* \mathrm{p}<0,05 * * \mathrm{p}<0,01$ Two-way ANOVA and Bonferroni post hoc test). 
Histaminergic neurotransmission as a gateway for the effects of Oleoylethanolamide

A

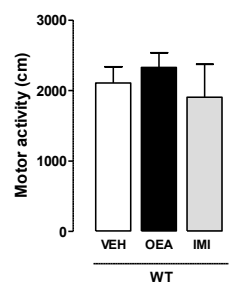

C

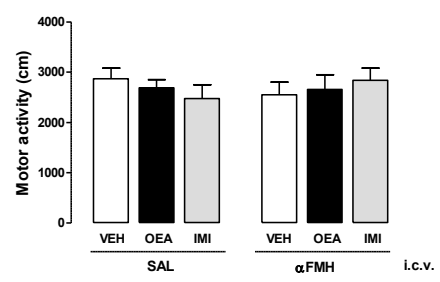

B

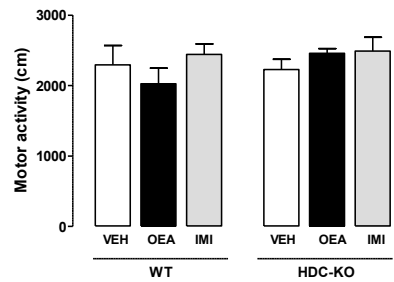

D

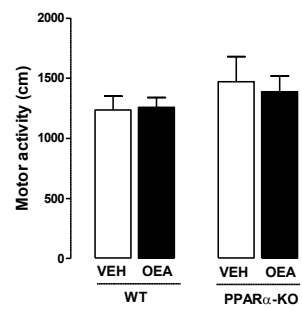

Figure 14. OEA systemic administration did not change general motor activity in normal, histamine deficient- and PPAR $\alpha-\mathrm{KO}$ mice in the open field test. (A) Effect of systemic administration of OEA $(10 \mathrm{mg} / \mathrm{kg}$ i.p. repeated regimen) or vehicle to WT and HDC-KO male mice $(\mathrm{n}=5-11)$. (B) Effect of systemic administration of OEA (5 or $10 \mathrm{mg} / \mathrm{kg}$ i.p. sub-chronic regimen) or vehicle to WT and HDC-KO male mice $(\mathrm{n}=5-11)$. (C) Effect of systemic administration of OEA $(10 \mathrm{mg} / \mathrm{kg}$ i.p. $)$ in saline or $\alpha$-FMH (5 $\mu$ g i.c.v.) treated CD1 mice $(n=8-11)$. (D) Effect of systemic administration of OEA (10 mg/kg i.p. sub-chronic regimen) or vehicle to WT and PPAR- $\alpha$ KO male mice $(n=5-11)$. (ANOVA and Newman- Keuls post hoc test). 


\section{A (WT MICE)}

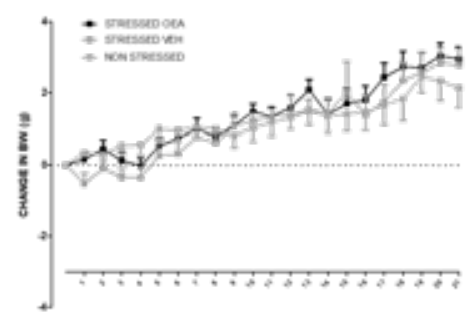

C(WT MICE)

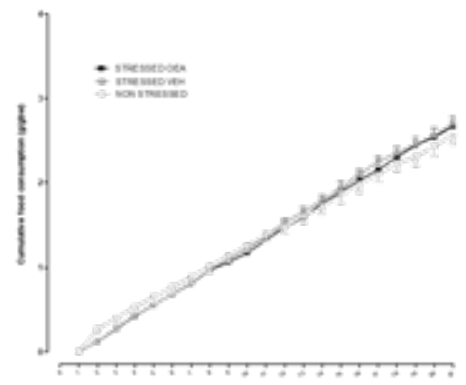

B (HDC-KO MICE)

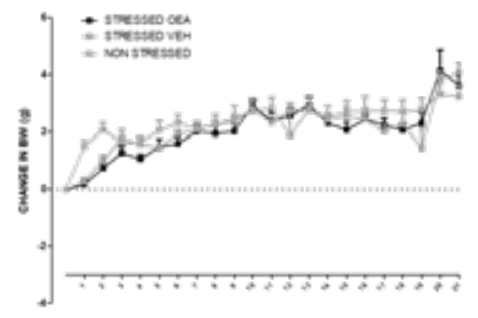

D (HDC-KO MICE)

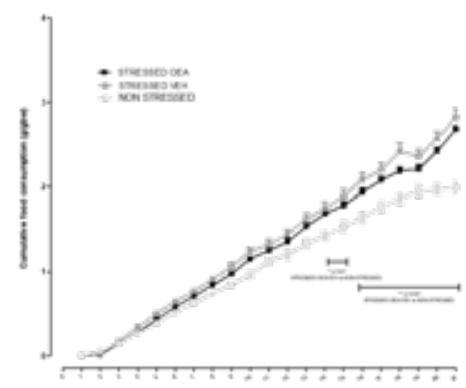

Figure15. Effects of the chronic social defeat stress on body weight and food consumption. CSDS (A) induced no significant increase in body weight in OEA and VEH treated stressed mice compared with non- $+6+$ stressed control group $(n=3-9)$. (B) No differences were detected in body weight between experimental group in HDC-KO mice ( $\mathrm{n}=6-7)$. (C) CSDS induced no significant increase in cumulative food consumption in OEA and VEH treated stressed mice compared with non-stressed control group $(n=3-9)$. (D) CSDS induced a significant increase in cumulative food consumption in OEA and VEH treated stressed HDC-KO mice compare to non stressed controls $(\mathrm{n}=6$ $7 * * \mathrm{p}<0.01 * * * \mathrm{p}<0.0001)$. 
Histaminergic neurotransmission as a gateway for the effects of Oleoylethanolamide

A ( WT MICE)

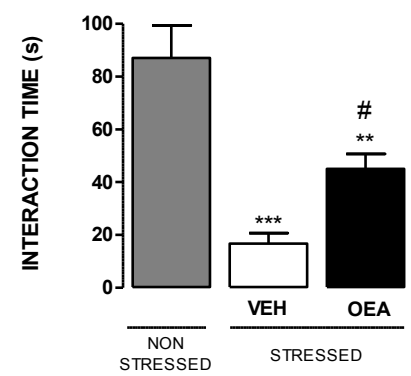

B ( HDC-KO MICE)

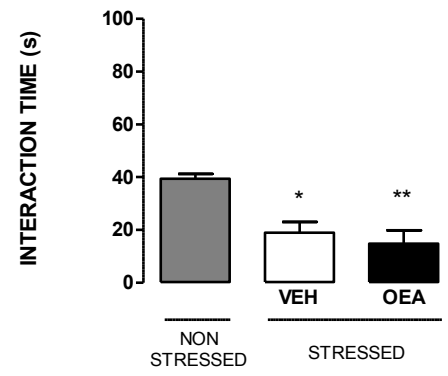

Figure 16. Social defeat stress induced social avoidance in WT and HDC-KO mice. (A) Defeated mice spent less time in the interaction compared to controls $(* * p<0.001$ OEA vs NON STRESSED; $* * * p>0.0001$ VEH vs NON STRESSED), OEA treatment increased interaction time in defeated animals compared to $\mathrm{VEH}$ treated controls $\left({ }^{\#} \mathrm{p}<0.05\right.$ OEA vs VEH). (B) HDC-KO defeated mice spent less time in the interaction compared to controls $(* \mathrm{P}<0.05 * * \mathrm{p}<0.001)$. OEA treatment did not increase interaction time in HDC-KO compared to controls. 
A (WT MICE)

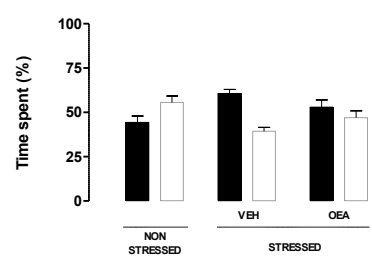

C (HDC-KO MICE)

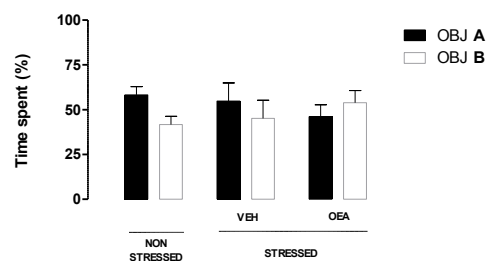

B (WT MICE)

- Familiar Object

$\square$ Novel Object

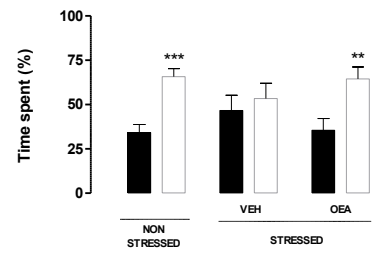

D (HDC-KO MICE)

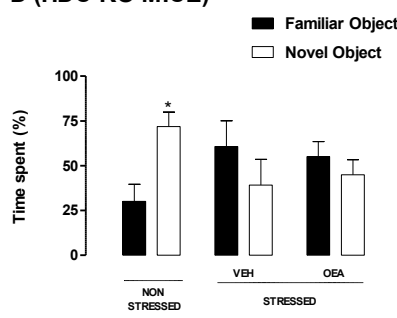

Figure 17. Effect of OEA on mice performances in the object recognition test. (A) Time spent in the exploration of Object A and B during T1 (One-way ANOVA and Bonferroni's MCT). (B) T2 was performed $1 \mathrm{~h}$ after training. Results are calculated as individual percentage of time spent exploring familiar (black columns) and novel (white columns) objects. One-way ANOVA and Bonferroni's MCT revealed an overall significant difference in the exploration of novel object compared to familiar one in non stressed controls and OEA treated stressed mice (means \pm S.E.M. of 3-9 animals per experimental group. ${ }^{* * * *} \mathrm{P}<0.0001 ;{ }^{* *} \mathrm{p}<0.01$, vs. respective familiar object). (C) Time spent in the exploration of Object $A$ and $B$ during T1 in HDC-KO mice (One-way ANOVA and Bonferroni's MCT). (D) Percentage of time spent exploring familiar (black columns) and novel (white columns) objects in stressed and non stressed control mice. One-way ANOVA revealed an overall significant difference in the exploration of novel object compared to familiar one in non stressed controls ( \pm SEM of 6-7 animals per experimental group. $\left.{ }^{*} \mathrm{p}<0.05\right)$. 
Histaminergic neurotransmission as a gateway for the effects of Oleoylethanolamide
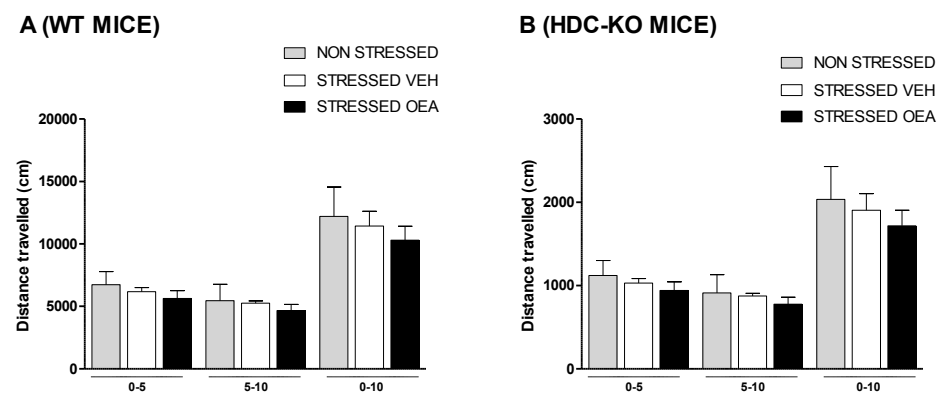

Figure 18. CSDS did not change general motor activity in normal and histamine deficient- mice in the open field test. (A) Effect of CSDS in WT animals treated with OEA (10 mg/kg i.p.) or vehicle compared to non stressed controls ( $n=3-7)$. (B) Effect of CSDS in HDC-KO animals treated with OEA $(10 \mathrm{mg} / \mathrm{kg}$ i.p. $)$ or vehicle compared to non stressed controls $(\mathrm{n}=6-7)$ (ANOVA and Bonferroni post hoc test). 
ANNO 2007

Bracardi M., La Materia e lo Spirito. Mario Ridolfi nel paesaggio umbro

Coppi E., Purines as Transmitter Molecules. Electrophysiological Studies on Purinergic Signalling in Different Cell Systems

Mannini M., Molecular Magnetic Materials on Solid Surfaces

Natali I., The Ur-Portrait. Stephen Hero ed il processo di creazione artistica in A Portrait of the Artist as a Young Man

Petretto L., Imprenditore ed Università nello start-up di impresa. Ruoli e relazioni critiche

ANNO 2008

Bemporad F., Folding and Aggregation Studies in the Acylphosphatase-Like Family

Buono A., Esercito, istituzioni, territorio. Alloggiamenti militari e «case Herme» nello Stato di Milano (secoli XVI e XVII)

Castenasi S., La finanza di progetto tra interesse pubblico e interessi privati

Colica G., Use of Microorganisms in the Removal of Pollutants from the Wastewater

Gabbiani C., Proteins as Possible Targets for Antitumor Metal Complexes: Biophysical Studies of their Interactions

ANNO 2009

Decorosi F., Studio di ceppi batterici per il biorisanamento di suoli contaminati da Cr(VI)

Di Carlo P., I Kalasha del Hindu Kush: ricerche linguistiche e antropologiche

Di Patti F., Finite-Size Effects in Stochastic Models of Population Dynamics: Applications to Biomedicine and Biology

Inzitari M., Determinants of Mobility Disability in Older Adults: Evidence from Population-Based Epidemiologic Studies

Macrì F., Verso un nuovo diritto penale sessuale. Diritto vivente, diritto comparato e prospettive di riforma della disciplina dei reati sessuali in Italia

Pace R., Identità e diritti delle donne. Per una cittadinanza di genere nella formazione

Vignolini S., Sub-Wavelength Probing and Modification of Complex Photonic Structures

ANNO 2010

Fedi M., "Tuo lumine». L'accademia dei Risvegliati e lo spettacolo a Pistoia tra Sei e Settecento

Fondi M., Bioinformatics of genome evolution: from ancestral to modern metabolism. Phylogenomics and comparative genomics to understand microbial evolution

Marino E., An Integrated Nonlinear Wind-Waves Model for Offshore Wind Turbines

Orsi V., Crisi e Rigenerazione nella valle dell'Alto Khabur (Siria). La produzione ceramica nel passaggio dal Bronzo Antico al Bronzo Medio

Polito C., Molecular imaging in Parkinson's disease

Romano R., Smart Skin Envelope. Integrazione architettonica di tecnologie dinamiche e innovative per il risparmio energetico

ANNO 2011

Acciaioli S., Il trompe-l' $e i l$ letterario, ovvero il sorriso ironico nell'opera di Wilhelm Hauff

Bernacchioni C., Sfingolipidi bioattivi e loro ruolo nell'azione biologica di fattori di crescita e citochine

Fabbri N., Bragg spectroscopy of quantum gases: Exploring physics in one dimension

Gordillo Hervás R., La construcción religiosa de la Hélade imperial: El Panhelenion

Mugelli C., Indipendenza e professionalità del giudice in Cina

Pollastri S., Il ruolo di TAF12B e UVR3 nel ciclo circadiano dei vegetali

Salizzoni E., Paesaggi Protetti. Laboratori di sperimentazione per il paesaggio costiero euro-mediterraneo 
ANNO 2012

Evangelisti E., Structural and functional aspects of membranes: the involvement of lipid rafts in Alzheimer's disease pathogenesis. The interplay between protein oligomers and plasma membrane physicochemical features in determining cytotoxicity

Bondì D., Filosofia e storiografia nel dibattito anglo-americano sulla svolta linguistica

Petrucci F., Petri Candidi Decembrii Epistolarum iuvenilium libri octo. A cura di Federico Petrucci

Alberti M., La 'scoperta' dei disoccupati. Alle origini dell'indagine statistica sulla disoccupazione nell'Italia liberale (1893-1915)

Gualdani R., Using the Patch-Clamp technique to shed light on ion channels structure, function and pharmacology

Adessi A., Hydrogen production using Purple Non-Sulfur Bacteria (PNSB) cultivated under natural or artificial light conditions with synthetic or fermentation derived substrates

Ramalli A., Development of novel ultrasound techniques for imaging and elastography. From simulation to real-time implementation

ANNO 2013

Lunghi C., Early cross-modal interactions and adult human visual cortical plasticity revealed by binocular rivalry

Brancasi I., Architettura e illuminismo: filosofia e progetti di città nel tardo Settecento francese

Cucinotta E., Produzione poetica e storia nella prassi e nella teoria greca di età classica

Pellegrini L., Circostanze del reato: trasformazioni in atto e prospettive di riforma

Locatelli M., Mid infrared digital holography and terahertz imaging

Muniz Miranda F., Modelling of spectroscipic and structural properties using molecular dynamics

Bacci M., Dinamica molecolare e modelli al continuo per il trasporto di molecole proteiche - Coarsegrained molecular dynamics and continuum models for the transport of protein molecole

Martelli R., Characteristics of raw and cooked fillets in species of actual and potential interest for italian aquaculture: rainbow trout (oncorhynchus mykiss) and meagre (argyrosomus regius)

ANNO 2014

Lana D., A study on cholinergic signal transduction pathways involved in short term and long term memory formation in the rat hippocampus. Molecular and cellular alterations underlying memory impairments in animal models of neurodegeneration

Lopez Garcia A., Los Auditoria de Roma y el Athenaeum de Adriano

Pastorelli G., L'immagine del cane in Franz Kafka

Bussoletti A., L'età berlusconiana. Il centro-destra dai poli alla Casa della Libertà 1994-2001

Malavolti L., Single molecule magnets sublimated on conducting and magnetic substrates

Belingardi C., Comunanze urbane. Autogestione e cura dei luoghi

Guzzo E., Il tempio nel tempio. Il tombeau di Rousseau al Panthéon di Parigi

ANNO 2015

Lombardi N., MEREAFaPS: uno Studio di Farmacovigilanza Attiva e Farmacoepidemiologia in Pronto Soccorso

Baratta L., "A Marvellous and Strange Event». Racconti di nascite mostruose nell'Inghilterra della prima età moderna

Richichi I.A., La teocrazia: crisi e trasformazione di un modello politico nell'Europa del XVIII secolo

Palandri L., I giudici e l'arte. Stati Uniti ed Europa a confronto

Caselli N., Imaging and engineering optical localized modes at the nano scale

Calabrese G., Study and design of topologies and components for high power density dc-dc converters

Porzilli S., Rilevare l'architettura in legno. Protocolli metodologici per la documentazione delle architetture tradizionali lignee: $i$ casi studio dei villaggi careliani in Russia 
ANNO 2016

Martinelli S., Study of intracellular signaling pathways in Chronic Myeloproliferative Neoplasms

Abbado E., "La celeste guida”. L'oratorio musicale a Firenze: 1632-1799

Focarile P., I Mannelli di Firenze. Storia mecenatismo e identità di una famiglia fra cultura mercantile e cultura cortigiana

Nucciotti A., La dimensione normativa dell'imprenditorialità accademica. Tre casi di studio sugli investigatori principali, i loro gruppi di ricerca e i fattori di innesco dell'imprenditorialità accademica

Peruzzi P., La inutilizzabilità della prestazione

Lottini E., Magnetic Nanostructures: a promising approach towards RE-free permanent magnets

Uricchio T., Image Understanding by Socializing the Semantic Gap

ANNO 2017

Valenti R., Cerebral Small Vessel Disease and Cerebral Amyloid Angiopathy: neuroimaging markers, cognitive features and rehabilitative issues

Starnini M., L'uomo tutto intero. Biografia di Carlo Livi, psichiatra dell'Ottocento

Verardi D., La scienza e i segreti della natura a Napoli nel Rinascimento: la magia naturale di Giovan Battista Della Porta

Minicucci G., Il dolo nella bancarotta. Alla ricerca della tipicità soggettiva della fattispecie patrimoniale

Pattelli L., Imaging light transport at the femtosecond scale: a walk on the wild side of diffusion

Egea Molines M.T., Etnobotánica en el Alto Valle del Reno (Toscana y Emilia-Romaña, Italia). Etnobotanica nell'Alta Valle del Reno (Toscana ed Emilia-Romagna, Italia)

Romano I.M., Pressione turistica sul Centro Storico di Firenze - sito UNESCO. Un modello per la valutazione dell'impatto percettivo

ANNO 2018

Costa A., Histaminergic neurotransmission as a gateway for the effects of the fat sensing molecule Oleoylethanolamide. Focus on cognition and stress-reactivity

Solera D., "Sotto l'ombra della patente del Santo Officio». I familiares dell'Inquisizione romana tra XVI e XVII secolo

Landi G., Secession and Referendum. A new Dimension of International Law on Territorial Changes?

Sacchetti A., La costituente libertaria di Camillo Berneri. Un disegno politico tra federalismo e anarchismo

Livi L.F., New quantum simulations with ultracold Ytterbium gases

Bellini E., Ambienti sensoriali "terapeutici” che rendano Abili. Un progetto integrato di vita per persone con Disturbi dello Spettro Autistico

Piscitelli L.R., Serviceability and post-failure behaviour of laminated glass structural elements 
